University of South Carolina

Scholar Commons

Theses and Dissertations

6-30-2016

\title{
Modeling Human-Structure Interaction Using A Controller System
}

Albert Ricardo Ortiz Lasprilla

University of South Carolina

Follow this and additional works at: https://scholarcommons.sc.edu/etd

Part of the Civil Engineering Commons

\section{Recommended Citation}

Lasprilla, A. O.(2016). Modeling Human-Structure Interaction Using A Controller System. (Doctoral dissertation). Retrieved from https://scholarcommons.sc.edu/etd/3484

This Open Access Dissertation is brought to you by Scholar Commons. It has been accepted for inclusion in Theses and Dissertations by an authorized administrator of Scholar Commons. For more information, please contact digres@mailbox.sc.edu. 
Modeling Human-Structure Interaction Using a Controller System

by

Albert Ricardo Ortiz Lasprilla

Bachelor of Science

Universidad del Valle 2008 and 2011

Master of Science

Universidad del Valle 2011

Submitted in Partial Fulfillment of the Requirements

for the Degree of Doctor of Philosophy in

Civil Engineering

College of Engineering and Computing

University of South Carolina

2016

Accepted by:

Juan M. Caicedo, Major Professor

Robert L. Mullen, Committee Member

Dimitris Rizos, Committee Member

Gabriel Terejanu, Committee Member

Lacy Ford, Senior Vice Provost and Dean of Graduate Studies 
(C) Copyright by Albert Ricardo Ortiz Lasprilla, 2016 All Rights Reserved. 


\section{DedicAtion}

This work would not have been possible without the help of my loving wife Tayrin. This is nothing compared to what you have done for me. I am eternally grateful.

I love you. 


\section{ACKNOWLEDGMENTS}

I am deeply grateful to my supervisor Dr. Juan M. Caicedo for opening the doors of the Structural Dynamics and Intelligent Infrastructure laboratory (SDII) at the University of South Carolina. My experience at SDII was exciting. The expectations related to my PhD were fulfilled. I also thank Dr. Caicedo for his motivation, positive feedback, and personal advice. He has my admiration and gratitude for the guidance and support throughout this research.

I am thankful to professors Dr. Robert L. Mullen, Dr. Gabriel Terejanu, and Dr. Dimitris Rizos for their time, comments, and suggestions for the realization of this work.

I would like to thank the institutions who financially contributed to this work: the University of South Carolina and the Foundation for the Future of Colombia (Colfuturo).

Many thanks to all students and staff of the structures laboratory who collaborated with tests. Special thanks to Ramin, Benjamin, Yohanna, Mus, Diego and Gustavo for the feedback and the endless discussions about the research and the methodology we used.

My deepest gratitude is to my parents, Carlos and Mary, and my brothers Carlos Eduardo and Daniel. I am happy to be member of this wonderful family.

To my wife, Tayrin and my son, Alejandro. I cannot thank you enough for making me happy every day of my life. 


\begin{abstract}
The effects of human loads on structures are difficult to predict because they depend on the type of activity people are performing. However, models for typical activities such as standing, sitting and jumping have been proposed in the literature. Traditional models represent the human body as a system of lumped masses, dampers and springs arranged in a system with multiple degrees of freedom. Arguably, these models might not fully represent the human body because lumped masses, dampers and springs cannot add energy to the overall system. Furthermore, people could react differently to different levels of excitation and other environmental conditions.

Controller systems have been widely used in electrical, seismic and other fields of engineering for systems in which setting a specific response is important. Given that the human acts like a controller system, where the feedback affects the response of the system, and the specific use of controllers is becoming common in structural engineering, this research developed a controller model to reproduce the phenomenon of Human-Structure Interaction (HSI). The methodology consisted in updating the parameters of the controller using experimental data from tests involving humans over a previously characterized structure. The controller system was the widely known Proportional, Integrative and Derivative (PID) controller and its derivations, PD and PI. Parameters of the controller were updated using a Bayesian probabilistic approach. Models were developed based on transfer functions obtained from experimental tests. A comparison of controller models and traditional Mass-Spring-Damper (MSD) models is performed at the end for validation purposes.
\end{abstract}




\section{TABle of Contents}

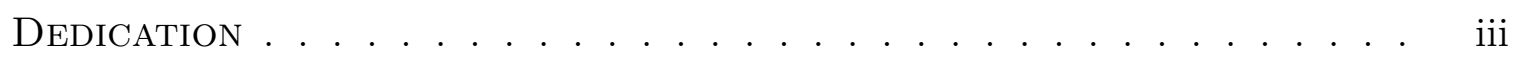

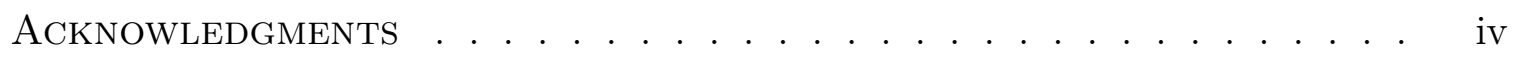

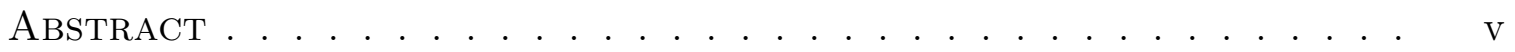

LIST OF TABLES . . . . . . . . . . . . . . . . . . viii

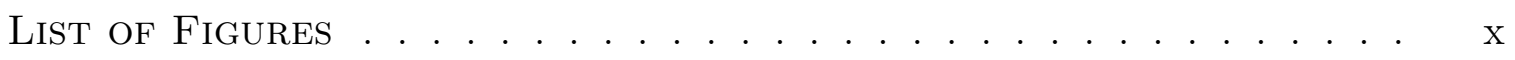

Chapter 1 Introduction $\ldots \ldots \ldots \ldots \ldots \ldots$

1.1 Traditional models used in HSI . . . . . . . . . . . . . 4

1.2 The human as a control system $\ldots \ldots \ldots \ldots \ldots \ldots$

Chapter 2 Methodologi . . . . . . . . . . . . . . . 9

2.1 Human-structure Interaction as a closed-loop control system $\ldots \ldots .9$

2.2 Bayesian Model Updating . . . . . . . . . . . . . . . 15

2.3 Probabilistic Model Selection _. . . . . . . . . . . . . 20

Chapter 3 EXPerimental testing AND UPdating of EMPTy StruCture 22

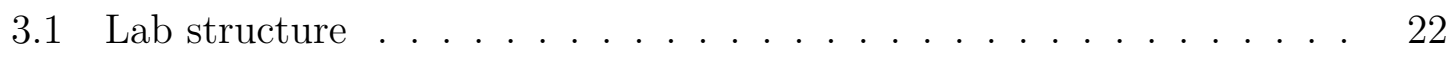

3.2 Instrumentation and tests $\ldots \ldots \ldots \ldots \ldots \ldots$

3.3 Parameters of the structure $\ldots \ldots \ldots \ldots \ldots \ldots$ 
3.4 Conclusion remarks . . . . . . . . . . . . . . . .

Chapter 4 Results . . . . . . . . . . . . . . . . 36

4.1 Parameter updating of the models . . . . . . . . . . . 36

4.2 Model selection . . . . . . . . . . . . . . . . . . . . 53

4.3 Controller models for groups of people $\ldots \ldots \ldots \ldots \ldots$

Chapter 5 Conclusions and future Work $\ldots \ldots \ldots \ldots$

5.1 Conclusions . . . . . . . . . . . . . . . . . . . . . . 59

5.2 Future Work . . . . . . . . . . . . . . . . . . 60

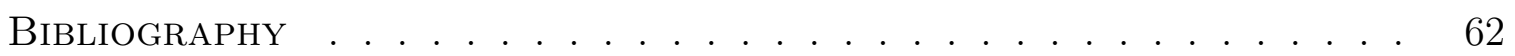

ApPendix A Stability Conditions . . . . . . . . . . . . . 69

A.1 PID controller . . . . . . . . . . . . . . . . . . . . . 69

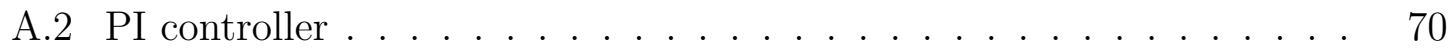

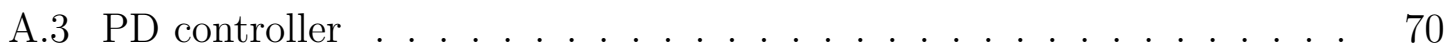

ApPendix B Convergence. . . . . . . . . . . . . . 71

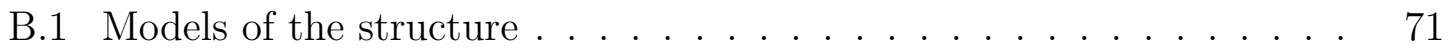

B.2 Models of human-structure interaction $\ldots \ldots \ldots \ldots \ldots$ 


\section{LIST OF TABLES}

Table 1.1 Influence of human occupation or a mass on the natural frequency and damping ratio of a slab . . . . . . . . . . . 3

Table 1.2 Characteristics of SDOF and MDOF models of a standing human occupant . . . . . . . . . . . . . . . . 7

Table 2.1 Models of the structure . . . . . . . . . . . . . . . . . . . 19

Table 2.2 Models of the human . . . . . . . . . . . . . . . . . . . . 19

Table 2.3 Models to be updated . . . . . . . . . . . . . . . . . . . . 19

Table 2.4 Kass and Raftery scale for Bayes factor . . . . . . . . . . . . . 21

Table 3.1 Prior PDFs used for updating the parameters . . . . . . . . . . . 28

Table 3.2 Prior PDFs used for updating the parameters . . . . . . . . . . . . 31

Table 4.1 Models to be updated . . . . . . . . . . . . . . . . . 37

Table 4.2 Prior PDFs of the structure's parameters . . . . . . . . . . . . . 38

Table 4.3 Prior definition for parameters of the PID, PI, and PD controller . 38

Table 4.4 Number of samples used and page location of convergence plots . . 38

Table 4.5 Moments of variables describing the parameters of models 1,2

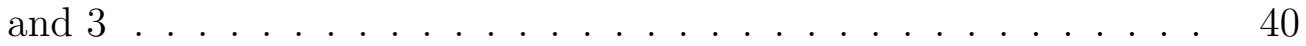

Table 4.6 Prior definition for parameters of the SDOF model of the human . 45

Table 4.7 Moments of random variables describing the parameters of the model 4 . . . . . . . . . . . . . . . . . 45

Table 4.8 Prior definition for parameters of the 2DOF model of the human . 48 
Table 4.9 Moments of random variables describing the parameters of the model $5 \ldots \ldots \ldots \ldots$. . . . . . . . . . . . . 4 48

Table 4.10 Moments of random variables describing the parameters of the model 5 (Cont.) . . . . . . . . . . . . . . . . . . 48

Table 4.11 Results of the integration of human-structure interaction models . 54

Table 4.12 Classificacion of human-structure interaction models for Bayes factor, $2 L n(K) \ldots \ldots \ldots \ldots \ldots \ldots \ldots$

Table 4.13 Classificacion for human-structure interaction models based on Kass and Raftery scale $[1] \ldots \ldots \ldots$. . . . . . . . . . 54

Table 4.14 Characteristics of the people involved in tests . . . . . . . . 55

Table 4.15 Moments of variables describing the PI controller for the people $\mathrm{P} 1, \mathrm{P} 2$ and $\mathrm{P} 3 \ldots \ldots \ldots \ldots \ldots \ldots$ 


\section{List OF Figures}

Figure 1.1 Single and two degree of freedom models commonly used for modeling human dynamics . . . . . . . . . . . . . . . . . . . . . 6

Figure 2.1 Block diagram of a closed-loop control system . . . . . . . . . . . 9

Figure 2.2 Physical representation of the closed-loop control system in an occupied structure . . . . . . . . . . . . . . . . . . . 10

Figure 2.3 Human-structure interaction controller model for a group of $n$

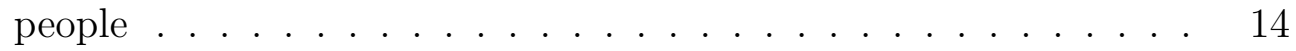

Figure 2.4 Geweke z-score for a variable . . . . . . . . . . . . . . 16

Figure 2.5 Geweke z-score for a variable . . . . . . . . . . . . . . . 17

Figure 2.6 Probabilistic model updating flow chart . . . . . . . . . . . . . 17

Figure 2.7 Flow chart for updating HSI models . . . . . . . . . . . . . . 18

Figure 3.1 Plant and side views of lab specimen . . . . . . . . . . . . 23

Figure 3.2 Test configurations: Empty structure . . . . . . . . . . . . . . . 24

Figure 3.3 Typical free vibration test performed at the structure . . . . . . . 25

Figure 3.4 Typical acceleration (left) and force (right) data recorded from the impact tests over the structure . . . . . . . . . . . 25

Figure 3.5 Experimental transfer function of the empty structure and data (bold dots) used for updating the configuration A . . . . . . . 27

Figure 3.6 Marginal histograms and samples generated to updated the SDOF model of the Configuration A . . . . . . . . . . . . . 29

Figure 3.7 Posterior predictive check of the Configuration A . . . . . . . . . 30 
Figure 3.8 Experimental transfer function of the empty structure and data (bold dots) used for updating the configuration B . . . . . . . 31

Figure 3.9 Marginal histograms and samples generated to update the Two DOF model of the Configuration B (Part 1 of 3 ) . . . . . . . 32

Figure 3.10 Marginal histograms and samples generated to update the Two DOF model of the Configuration B (Part 2 of 3 ) . . . . . . . 33

Figure 3.11 Marginal histograms and samples generated to update the Two DOF model of the Configuration B (Part 3 of 3) . . . . . . . 34

Figure 3.12 Posterior predictive check of the Configuration B . . . . . . . 34

Figure 4.1 Empty and occupied transfer functions of the structure configuration $\mathrm{A} \ldots \ldots \ldots \ldots . \ldots \ldots$

Figure 4.2 Marginal histogram (diagonal) and samples for PID controller parameters . . . . . . . . . . . . . . . 39

Figure 4.3 Marginal histogram (diagonal) and samples for PI controller parameters . . . . . . . . . . . . . . . . . 39

Figure 4.4 Marginal histogram (diagonal) and samples for PD controller parameters . . . . . . . . . . . . . . . 40

Figure 4.5 Samples for PID controller and structure's parameters _ . . . . . 41

Figure 4.6 Samples for PI controller and structure's parameters _ . . . . . . 41

Figure 4.7 Samples for PD controller and structure's parameters _ . . . . . 42

Figure 4.8 Posterior predictive check of model $1 \ldots \ldots$. . . . . . . . . . 43

Figure 4.9 Posterior predictive check of the model $2 \ldots \ldots$. . . . . . . 43

Figure 4.10 Posterior predictive check of the model $3 \ldots \ldots$. . . . . . . . 44

Figure 4.11 Marginal histogram (diagonal) and samples for the SDOF model of the human . . . . . . . . . . . . . . . 46

Figure 4.12 Samples for SDOF model of the human and structure's param-

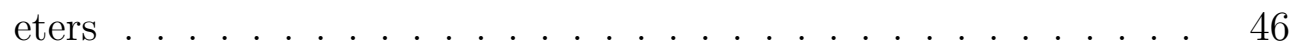


Figure 4.13 Posterior predictive check of the model $4 \ldots \ldots$. . . . . . . . . . 47

Figure 4.14 Marginal histogram (diagonal) and samples for the 2DOF model of the human (Figure 1 of 3 ) . . . . . . . . . . . . 49

Figure 4.15 Marginal histogram (diagonal) and samples for the 2DOF model of the human (Figure 2 of 3 ) $\ldots \ldots \ldots \ldots$

Figure 4.16 Samples for the 2DOF model of the human (Figure 3 of 3 ) $\ldots 50$

Figure 4.17 Samples for 2DOF model of the human and structure parameters $($ Figure 1 of 2$) \ldots \ldots \ldots \ldots \ldots \ldots$

Figure 4.18 Samples for 2DOF model of the human and structure parameters (Figure 2 of 2 . . . . . . . . . . . . . . 51

Figure 4.19 Posterior predictive check of the model $5 \ldots \ldots \ldots$. . . . . . 52

Figure 4.20 Converegence of the integration . . . . . . . . . . . 53

Figure 4.21 Posterior predictive checks of PI models for each single occupant . 57

Figure 4.22 Predictive checks for groups of two humans . . . . . . . . . . 58 


\section{Chapter 1}

\section{INTRODUCTION}

In the last century, structural engineers focused their attention on the design of structures able to withstand large loads induced by natural and humans hazards, such as earthquakes, high winds caused by tornadoes or hurricanes, flooding, and explosions. The strengthening of the structural engineering field on the design of structures against these hazards was outstanding. While a century ago many of these hazards were not well understood and empirical methods were used in order to prevent the collapse of many structures, today's infrastructure is a lot more resilient due to, in part, the design methodologies and new knowledge about hazards, materials, construction methods, etc.

In the way that new materials were developed, new structural concepts of structures' designs were also implemented by architects. The parallel and dependent evolution of both fields led to unimaginable structures where open spaces play a key role. This conception has been applied not only in the design of residential houses, but also in the construction of huge structures such as stadiums, dance floors, gyms, or lobby hotels; where the new materials, characterized as slender and lightweight, can be used. However, as new materials decrease concerns related to strength, excessive vibrations appear as one of the biggest challenges that the field of structural engineering is facing. Therefore, serviceability problems are indisputably a side effect of the new design tendencies.

In the study of serviceability conditions, the structures occupied by humans are of special interest, mainly because human comfort is the main target in the design, 
and their satisfaction could not be secured if vibration problems occur. In this way, problems related to the vibrations induced and felt by humans have attracted the attention as a current research topic in structural engineering.

The best example of vibrations induced by humans can be found in the UK. During its opening in 2000, the Millennium footbridge, a structure that cost $£ 18.7 \mathrm{M}$, showed excessive vibrations. The bridge was closed two days after opening. Repairs left the bridge closed until 2002 when, after a retrofit and investment from ARUP Inc. of $£ 5 \mathrm{M}$, the bridge was in-service again. Studies reported by Dallard et. al. [2] and Fitzpatrick et. al. [3] found that the vibrations were produced because of a feedback phenomenon that is rarely studied. This sketch of the problem has not been developed further. Some studies suggest, though, that the problem is because there is a coupling between the lateral frequency of the bridge and the lateral frequency induced by humans while walking, a typical resonance phenomenon. However, the case of resonance does not explain how the movement of the bridge induced people to change their step frequency and the load they applied. This problem is considered the formal starting point of human-structure interaction studies. More structural problems caused by humans have been reported in Morris [4], Dallard et. al. [2], Fitzpatrick et. al. [3], Bodare et. al. [5], and Macdonald [6].

Another effect of the human-structure interaction is observed when people are in a passive condition like sitting or standing over the structure. Ortiz et. al. [7] found that the properties of the structure change significantly when a structure is occupied by humans. In their tests, they found that a mass produced by bags of sand could not represent the phenomena. Falati et. al. [8] performed similar tests, but on a simple supported slab. They found interesting changes in the frequency, but especially in the damping ratio. Table 1.1 shows the change in the damping ratio and the frequency for the slab's main mode of vibration. Values reported in Table 1.1 are all deterministic. The probability distribution and the correlation of the model's 
parameters have not been reported in the literature.

Table 1.1: Influence of human occupation or a mass on the natural frequency and damping ratio of a slab, from [8]

\begin{tabular}{ccc}
\hline Configuration & $\begin{array}{c}\text { Natural Frequency } \\
{[\mathrm{Hz}]}\end{array}$ & $\begin{array}{c}\text { Damping Ratio } \\
{[\%]}\end{array}$ \\
\hline empty structure & 8.0 & 1.1 \\
one human occupant $[75 \mathrm{~kg}]$ & 7.9 & 3.8 \\
equivalent mass of one man $[75 \mathrm{~kg}]$ & 7.7 & 1.4 \\
\hline empty structure with two screed layers & 10.1 & 1.2 \\
one human occupant & 10.0 & 3.1 \\
two human occupants & 10.0 & 3.5 \\
equivalent mass of one occupant & 9.9 & 1.3 \\
equivalent mass of two occupants & 9.8 & 1.3 \\
\hline
\end{tabular}

Similar studies to those above compared the effect of people on structures. Results from these studies support evidence that humans add damping and stiffness to the structure rather than only mass $[9,10,11]$. Some studies also suggest that people in a passive condition (without movement) strongly modified the dynamics of the structure while the people in an active condition do not [12]. These conclusions suggest that people in a passive condition should be modeled differently than in the traditional mass model.

Different models of the human body have been developed in order to predict the behavior and the interaction of humans with other objects such as cars, aircrafts and civil structures. Models found in the literature are based on modeling the human as a set of lumped masses, dampers, and springs (called MDS models). Beyond the development of MDS models, the use of these parameters should be evaluated to include a stronger feedback action because the nature of the human. The variety of MDS models and their parameters' values produce concerns, which are commonly attached to individuals employed and tests conditions like vibration levels [13], however the variability of MDS models also means that further work is necessary to develop better models even from the traditional ones based on single and multiple degrees-of-freedom 
systems (SDOF and MDOF).

\subsection{Traditional MOdels USED IN HSI}

A literature review published by Zivanovic, Pavic, and Reynolds [14] indicates that there are two different types of models for the human body. The first and more general is called the mass model [15]. This type of model only uses the humans' mass and does not model any HSI. This model is commonly used in building codes such as the International Building Code (IBC) [16] and the guidelines proposed by the American Society of Civil Engineers (ASCE) $[15,17]$. The second type of model is based on modeling the human as a mechanical system composed of lumped masses, dampers, and springs (MDS) systems, which are likely used in problems related to human-structure interaction. Many MDS models have been developed, ranging from single to multiple degrees of freedom, in order to represent the dynamics of the human body $[8,18,19,20,21,22]$.

MASS MODELS FOR HUMANS

Current design codes specify the application of live loads for structures occupied by people $[15,16]$. For example, the ASCE 7-05 code [15] specifies a load for residential use of $30 \mathrm{ps} f\left(1.44 \mathrm{kN} / \mathrm{m}^{2}\right)$, however the load is 3.3 times higher when the structure is a gymnasium, dance hall, or a stadium, when the minimum design load is specified as $100 p s f\left(4.49 k N / m^{2}\right)$.

A higher load is justified because of the high density of people and the possible amplification that dynamic forces induced by humans may induce over the structure. However, this consideration does not take into account additional changes over the structure, such as different damping ratios, or stiffness changes, which may result in a modification of the natural frequency of the structure, as seen before in Table 1.1. 
Current design methodologies involve the application of Load Resistance and Design Factors, (LRFD) methodology, which means that the load is amplified by a load factor, which is usually around 1.6 for live load. The high magnitude of the live load clearly states the importance of this type of load, but this does not consider the human-structure interaction, causing over-design in some cases and excessive vibration in others.

The serviceability limit state is the second condition limit set in guidelines. This focuses the attention in the human perception and their comfort, in order to avoid the panic, or feeling of discomfort related to the dynamic response of the structure. Serviceability conditions are based on structural accelerations. Limits are found in different guidelines as a function of the type of structure and natural frequency of the floor. $[23,24]$

\section{MDS Models For Humans}

Mass-damper-spring models are used in HSI problems for modeling the human body [13]. These models allow the interaction between dynamic properties of the human body and the structure through inducing an action-reaction force. Researchers find these models useful because they can reproduce a dynamic force induced by humans as a function of the mass, damping, and stiffness of the body. The Joint Working Group, from the Institution of Structural Engineers in the UK, is the only institution which has formally proposed MDS models for guidelines [25]. This guide, "Dynamic performance requirements for permanent grandstands subject to crowd action", recommended values for modeling passive people in grandstands in its latest edition. The proposed models take into account the stiffness and damping beyond the mass of the spectators.

The mathematical formulation of MDS models follows the theory of structural dynamics. Independent of the number of degrees of freedom, the dynamic properties 
of the model can be easily represented as deterministic values. Many researchers have proposed values for the MDS parameters for HSI problems. Additional background of these models can be found in Griffin et. al. [26], and Zivanovic et. al. [14]. Figure 1.1 shows two of the models traditionally used in HSI problems.
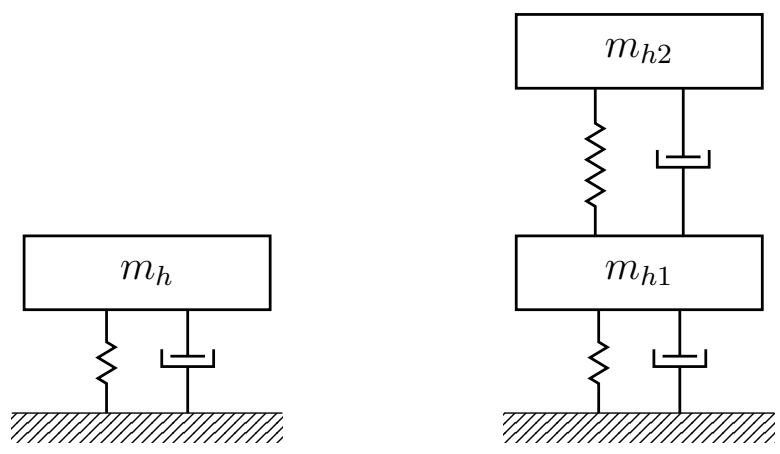

Figure 1.1: Single and two degree of freedom models commonly used for modeling human dynamics

Values for the parameters of the SDOF model were proposed by Griffin et al [22], Falati et. al. [8], and Brownjohn et al [19] in a deterministic fashion. The model parameters are described by single values of the damping constant $\left(c_{1}\right)$, mass $\left(m_{1}\right)$, and stiffness $\left(k_{1}\right)$. Equation 1.1 shows the response of the human system for an excitation, $p(t)$.

$$
m_{h} \ddot{x}(t)+c_{h} \dot{x}(t)+k_{h} x(t)=p(t)
$$

The equation describing the dynamics of a $2 \mathrm{DOF}$ is the same as equation 1.1, but the terms are replaced by matrices of mass, damping, and stiffness.

$$
M=\left[\begin{array}{cc}
m_{1} & 0 \\
0 & m_{2}
\end{array}\right], \quad C=\left[\begin{array}{cc}
c_{1}+c_{2} & -c_{2} \\
-c_{2} & c_{2}
\end{array}\right], \quad K=\left[\begin{array}{cc}
k_{1}+k_{2} & -k_{2} \\
-k_{2} & k_{2}
\end{array}\right]
$$


A summary of the variables for the SDOF and MDOF models used for modeling the human is shown in table 1.2. Models are taken from $[8,22,27,20,19]$.

Table 1.2: Characteristics of SDOF and MDOF models of a standing human occupant from $[8,20,27,22,19]$

\begin{tabular}{|c|c|c|c|}
\hline \multicolumn{2}{|c|}{ SDOF models } & \multicolumn{2}{|r|}{ 2DOF models } \\
\hline $\begin{array}{l}\text { Human } \\
\text { Model }\end{array}$ & Properties & $\begin{array}{l}\text { Human } \\
\text { Model }\end{array}$ & Properties \\
\hline Falati [8] & $\begin{array}{l}m_{h}=m_{t} / 3(\mathrm{~kg}) \\
k_{h}=107(\mathrm{kN} / \mathrm{m}) \\
c_{h}=1.636(\mathrm{kNs} / \mathrm{m})\end{array}$ & $\begin{array}{l}\text { Farah } \\
{[27]}\end{array}$ & $\begin{array}{l}m_{h 1}=74.4, m_{h 2}=7.3(\mathrm{~kg}) \\
k_{h 1}=149.2, k_{h 2}=15.4(\mathrm{kN} / \mathrm{m}) \\
c_{h 1}=2.85, c_{h 2}=0.086(\mathrm{kNs} / \mathrm{m})\end{array}$ \\
\hline $\begin{array}{l}\text { Brownjohn } \\
{[19]}\end{array}$ & $\begin{array}{l}m_{h}=m_{t}(\mathrm{~kg}) \\
k_{h}=82(\mathrm{kN} / \mathrm{m}) \\
c_{h}=1.946(\mathrm{kNs} / \mathrm{m})\end{array}$ & $\begin{array}{l}\text { ISO } 5982 \\
{[20]}\end{array}$ & $\begin{array}{l}m_{h 1}=62, m_{h 2}=13(\mathrm{~kg}) \\
k_{h 1}=62, k_{h 2}=80(\mathrm{kN} / \mathrm{m}) \\
c_{h 1}=1.46, c_{h 2}=0.93(\mathrm{kNs} / \mathrm{m})\end{array}$ \\
\hline $\begin{array}{l}\text { Griffin } \\
{[22]}\end{array}$ & $\begin{array}{l}m_{h}=74.2(\mathrm{~kg}) \\
k_{h}=96.5(\mathrm{kN} / \mathrm{m}) \\
c_{h}=3.71(\mathrm{kNs} / \mathrm{m})\end{array}$ & $\begin{array}{l}\text { Griffin } \\
{[22]}\end{array}$ & $\begin{array}{l}m_{h 1}=41.3, m_{h 2}=28.4(\mathrm{~kg}) \\
k_{h 1}=316.1, k_{h 2}=39.8(\mathrm{kN} / \mathrm{m}) \\
c_{h 1}=2.67, c_{h 2}=0.85(\mathrm{kNs} / \mathrm{m})\end{array}$ \\
\hline
\end{tabular}

\subsection{The HUMAN AS A CONTROL SYSTEM}

The concept of the human as a control system is not new. Giovanni Borelli (16081679), known as the father of modern biomechanics [28], related the anatomy of humans to mechanical systems 400 years ago. Psychological and medical fields have also studied the human body system [29, 30].

Firsts applications in the modern era focused on understanding the behavior of the brain and its connectivity to the motor system of the body. The popularization of biomechanical science in the last century enabled to in-depth research on the control skills associated with the human body for balance keeping [31]. The control approach of the human body has also been studied for understanding the change of the stability in an elderly population. For example, Melzer et. al. [32] studied the deterioration of the control skills for maintaining the balance of the body's center of mass.

Controller models based on the performance of the human body have also been applied to robotics for keeping stability at different positions, such as sitting, standing, 
walking and jumping [33, 34]. However, the short time response of the body to external loads is a challenge and has not been totally achieved, therefore, optimization techniques and faster controllers for modeling the body mechanics and body control loops actions are still under development.

The most common biomechanical model of the human body is the inverted pendulum system [35]. Controller models have been applied to this model in order to recreate the control loop between the input sensors (vestibular, visual, and proprioceptive) and the motor output. In their work, Hidenura and Jiang, develop a PID model for human balance keeping [36]. The closed-loop model was updated from a deterministic point of view, in the time domain. Results of their investigation suggested that the derivative factor $\left(K_{D}\right)$ of the PID controller plays a key role in the balance and the stability of the body.

Recent research developed by Bocian et. al. [37] used the inverted pendulum model for modeling the force induced by pedestrians over a vertically oscillating structure. The changes in damping induced by pedestrians over the structure, such as the changes seen in the Millenium bridge, could be addressed through this model. However, improvements to the model are suggested, specially focusing on the human system, which could lead to introduce the control action.

Even though considerable work has been done to model specific tasks done by humans using control theory, the application of controller for HSI is still at its fancy. The use of control theory in HSI introduces an alternative way for understanding the behavior of the human body, and its influence in the structure properties. This research will provide probabilistic information about the controllers that could be used for modeling the phenomenon. The traditional single and multiple degree of freedom models will be updated, and then compared with controller models. 


\section{Chapter 2}

\section{Methodology}

\subsection{Human-Structure Interaction as a ClOSED-LOOP CONTROL SYSTEM}

The idea of using control theory for modeling human-structure interaction comes from the fact that the feedback provided by MDS models is not adequate to correctly model the human-structure interaction. MDS models provide forces proportional to the relative velocity and displacement between the structure and the mass of the human. Closed-loop control systems provide more flexibility. The term closed-loop control implies the use of feedback in order to reduce system error [38]. Figure 2.1 shows a block diagram of a closed-loop control system where G(s) is transfer function of the structural system (or plant) and $\mathrm{H}(\mathrm{s})$ is the transfer function of the controller. The terms $\mathrm{R}(\mathrm{s})$ and $\mathrm{C}(\mathrm{s})$ represent the forces acting on the structure and the outputs of the system. $\mathrm{B}(\mathrm{s})$ is the force used to control the structure and $\mathrm{E}(\mathrm{s})$ is the actuating error signal.

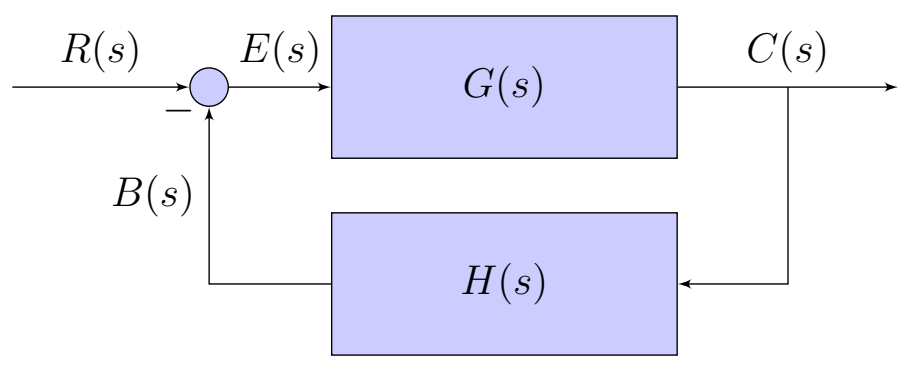

Figure 2.1: Block diagram of a closed-loop control system

The transfer function, $\mathrm{TF}(\mathrm{s})$, for a closed-loop control system as shown in fig- 
ure 2.1 is defined as:

$$
T F(s)=\frac{G(s)}{1+G(s) H(s)}
$$

where the terms $\mathrm{G}(\mathrm{s})$ and $\mathrm{H}(\mathrm{s})$ are, as mentioned before, mathematical representations of the plant and the controller in the Laplace domain, $s$. In this work, the structure is represented by the plant, $G(s)$, and the human by the controller, $H(s)$. It is assumed that the human is controlling the acceleration of the structure. Figure 2.2 shows a picture representing both sub-systems. The structure is a laboratory specimen composed of a cantilever beam and lumped masses, as described later in chapter 33. The controller describes the human, and models the dynamic force applied to the structure.

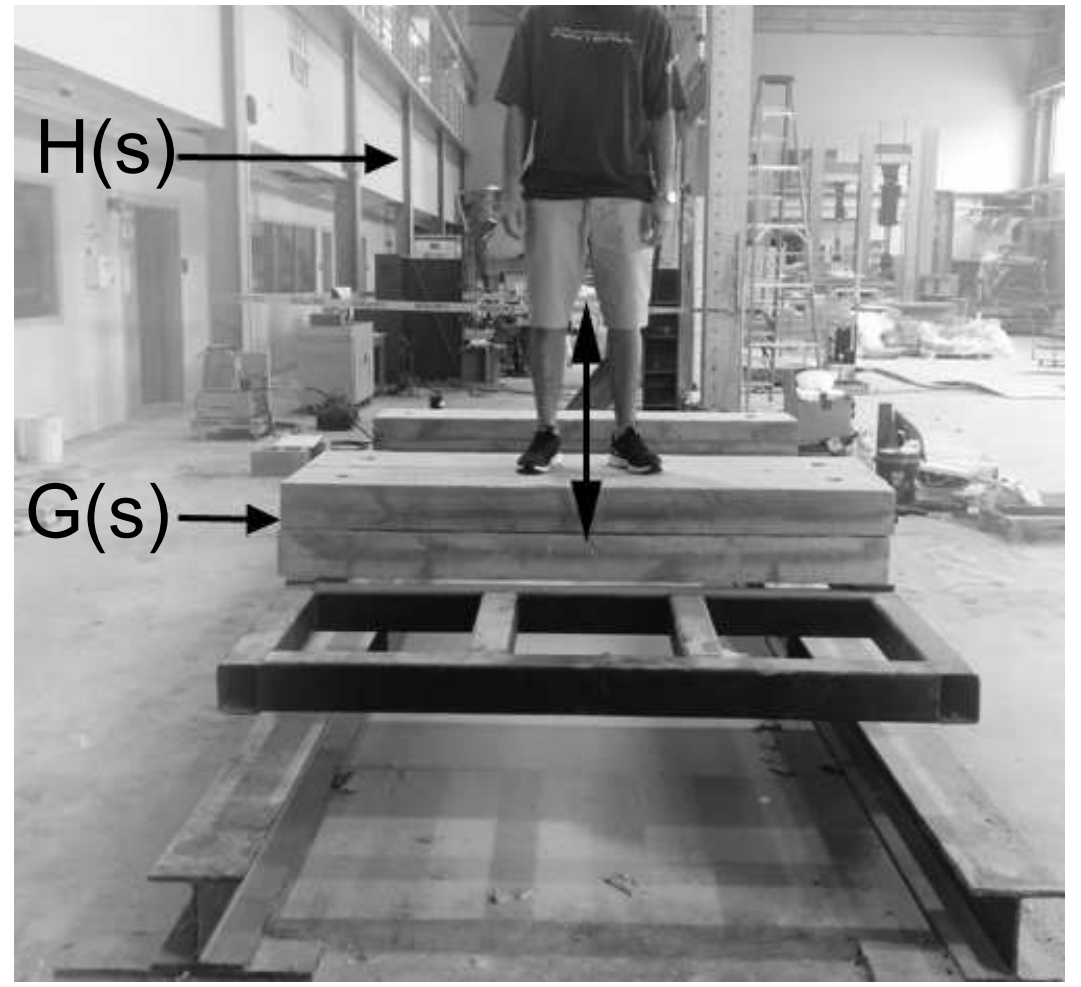

Figure 2.2: Physical representation of the closed-loop control system in an occupied structure

Models of the structure and the controller representing the human may be gener- 
ated in order to represent the interaction. The next two sections discuss in detail the models used for the structure and the controller.

MODELING THE STRUCTURE

The structure $G(s)$ can be modeled as a single or multiple degree of freedom system. Modeling errors are expected when modeling any type of structural system. There are errors due to the fact that the model does not accurately describe the behavior of the structure. Furthermore, uncertainty in the parameters of the model might be present. Depending on the complexity of the structure, modeling errors can be significant. The structural system considered for this work (discussed in detail in chapter 3) was specifically considered because of its simplicity, minimizing the chances of including modeling errors in the structural system, and allowing the study of closed-loop control theory in a controlled environment. In its simplest form, the structure can be modeled as a SDOF. For a SDOF, the term $\mathrm{G}(\mathrm{s})$ is defined as:

$$
G(s)=\frac{\frac{s^{2}}{m_{s}}}{s^{2}+\frac{c_{s}}{m_{s}} s+\frac{k_{s}}{m_{s}}}
$$

where the parameters $m_{s}, c_{s}$, and $k_{s}$ are the equivalent mass, damping coefficient, and stiffness of the structure when assumed to be an SDOF system, therefore the parameters of the model are $\Theta_{s}=\left\{m_{s}, c_{s}, k_{s}\right\}$. If the structure is modeled as a multiple degree of freedom system the parameters $m_{s}, c_{s}$, and $k_{s}$ become matrices. An alternative way for modeling the structure is using the poles and zeros of the system [39]. The transfer function of the structure can be expressed using the equation 2.3:

$$
G(s)=K s^{2} \frac{\left(s-z_{1}\right)\left(s-z_{2}\right) \ldots\left(s-z_{m-1}\right)\left(s-z_{m}\right)}{\left(s-p_{1}\right)\left(s-p_{2}\right) \ldots\left(s-p_{m-1}\right)\left(s-p_{m}\right)}
$$

In this equation, the poles, $p_{i}$, are roots of the denominator and the zeros, $z_{i}$, are roots of the numerator and $K$ is the gain. Each pole of the system contains information about the natural frequency, $\omega_{i}$, of the structure and its corresponding 
damping ratio, $\zeta_{i}$. In a structural system poles are complex conjugates and the relationship with the $i$-th natural frequency and associated damping ratio is:

$$
p_{i}=-\zeta_{i} \omega_{i} \pm \sqrt{\left(\zeta_{i} \omega_{i}\right)^{2}-\omega_{i}^{2}}
$$

Therefore, the model of the structure can be expressed in terms of natural frequencies, $\omega_{i}$; damping ratios, $\zeta_{i}$; and the gain, $K$. For a model with two poles and one zero, the parameters used in the model are $\Theta_{s}=\left\{\omega_{1}, \omega_{2}, \zeta_{1}, \zeta_{2}, \omega_{z 1}, \zeta_{z 1}, K\right\}$. An in-depth discussion of systems modeling using this approach can be found in [39] and $[38]$.

\section{Modeling THE CONTROLleR}

The controller is a decisive part in the closed-loop system. It is a device which monitors and physically alters the operating conditions of a given dynamical system [40]. For this research, the controller, $H(s)$, alters the operating conditions of the structure, $G(s)$. For example, a person standing on a structure will minimize the vibrations of a structure $[41,11,13]$. The use of controllers has become popular in the last century, boosted by the development of computers. Application of controllers involves almost all fields of engineering, such as, aerospace [42], biomechanical [43], electrical [44], or civil engineering [45]. One of the most common controllers it the Proportional, Integrative and Derivative (PID) and its derivation, PI and PD. PID controllers are used for controlling autonomous cars [46], for controlling the amount of glucose in the blood for ill patients [47], and for the control of Unmaneed Aerial Vehicles (UAV) [48] among others.

PID controllers use three parameters associated to the state of the error. The three parameters are the proportional term, $K_{p}$; the integrative term, $T_{i}$; and the derivative term, $T_{d}$. While the proportional term, $K_{p}$, multiplies the feedback, the 
integrative, $T_{i}$, and derivative, $T_{d}$, multiply the integral and derivative of the feedback. The transfer function for the PID is:

$$
H(s)=K_{p}\left(1+T_{d} s+\frac{1}{T_{i} s}\right)
$$

For a PD controller, the transfer function can be represented as:

$$
H(s)=K_{p}\left(1+T_{d} s\right)
$$

For a PI controller, the transfer function can be represented as:

$$
H(s)=K_{p}\left(1+\frac{1}{T_{i} s}\right)
$$

The model defined in equation 2.1 assumes the use of one controller for each human. Under this assumption, each person acts as an independent controller, commanding a control force on the structure. In the case of groups of people, the $i$-th controller, $H_{i}(s)$, represent a specific person, $(i)$. Figure 2.3 shows a block diagram of the whole human-structure interaction system when the structure is occupied by $n$ people acting independently. It is assumed that controllers are acting in parallel, indicating that the motion of one person has little effect on the motion of the next person, which is reasonable for standing people.

While this model should be appropriated to model humans acting in sync because of interactions with the structural system, it will not model interaction between humans (i.e. people pushing each other). Additional feedback loops could be formulated to model these interactions.

Therefore, the transfer function of the full HSI system occupied by $n$ independently people can be presented as: 


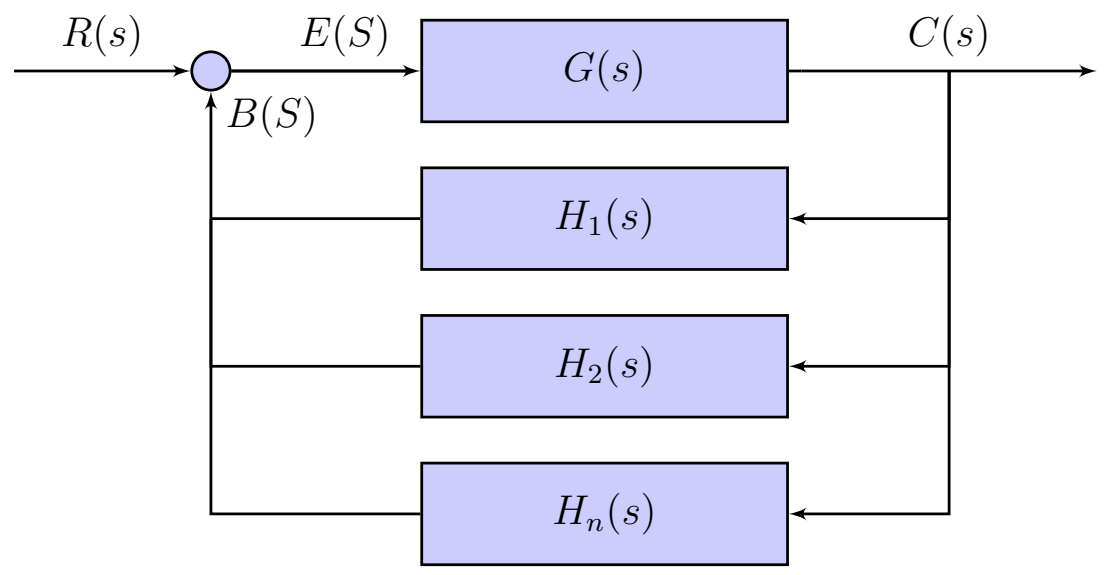

Figure 2.3: Human-structure interaction controller model for a group of $n$ people

$$
T F(s)=\frac{G(s)}{1+G(s)\left[H_{1}(s)+H_{2}(s)+\ldots+H_{n}(s)\right]}
$$

The stability of the closed-loop control models is the main concern in control design. The selection of the controller parameters must follow additional conditions in order to guarantee a finite response of the system given a finite excitation. In this research, the human-structure interaction is assumed stable, however, several cases in the literature suggest to model the interaction using an unstable system, for example, vandal loading over structures as footbridges [49], or pedestrians walking during the opening of the London Millennium bridge [50].

Analytical and graphical methods exist in the literature to check the system stability [38]. In this research, an analytical method, known as the Routh's stability criterion, is used. This is a mathematical criterion which consists in checking if the real part of the poles of a polynomial are all negative. A detailed explanation about the Routh's criterion is given by Ogata [38]. The stability conditions for the humanstructure interaction systems conform by the structure, $G(s)$, and the controllers, $H(s)$, are given in Appendix A. 


\subsection{Bayesian Model Updating}

The dynamic behavior of the models presented in the previous section depends on their parameters. In this research, Bayesian inference is used to update the parameters of the models based on experimental data [51, 52]. Bayesian inference is based on the Bayes theorem:

$$
P\left(\Theta \mid D, M_{j}\right)=\frac{P\left(D \mid \Theta, M_{j}\right) P\left(\Theta \mid M_{j}\right)}{P(D)}
$$

where $P\left(\Theta \mid D, M_{j}\right)$ is the posterior probability density function (PDF) of the parameters $\Theta$, for model $M_{j}$, given the observation $D . P\left(\Theta \mid M_{j}\right)$ is the prior PDF of the parameters $\Theta$ and it represents the knowledge of the parameters before updating. $P\left(D \mid \Theta, M_{j}\right)$ is the likelihood of the occurrence of the measurement $D$ given the vector of parameters $\Theta$ and model $M_{j} . P(D)$ is the probability of the observation D.

It is important to highlight some of the main differences between inference using classical methods and Bayesian inference. Classical methods assume that here is one underlining "true" probability distribution describing the parameters and the experimental data are random draws from this distribution. Bayesian inference is different. Here, the experimental data, or observations, are "fixed" and the probability density function is calculated given these observations. Therefore, it is important to highlight that the results of this research could change if other populations are used for the experiments. However, the overall procedure and concepts developed here should be applicable.

Several techniques are available to extract useful information from the posterior PDF. For example, one can calculate the Maximum A Posteriory (MAP) as a point estimate of the parameters. In this research, samples of the posterior are obtained to derive statistics of the parameters, investigate correlations, and fully describe their probability density functions. The samples are generated using the Markov chain 
Monte Carlo (MCMC) methodology [53, 54, 55]. The MCMC is a derivation of the Monte carlo sampling algorithm, where the samples distribution is based on an equilibrium condition. The Markov chain algorithm defines the probability of the next step based on the probability of the current step. In particular, the Metropolis algorithm was used. The convergence of the chains was checked using the method proposed by Geweke et. al. [56] and called Geweke z-score, which consist in comparing the mean and variance of different segments of a single chain. Figures 2.4 and 2.5 show an example of a Geweke score for two parameters. Figures 2.4 is showing convergence because the z-score is within 2 standard deviations. In Figure 2.5, the parameter has deviations exceeding 2 standard deviations, suggesting that additional samples are required for convergence.

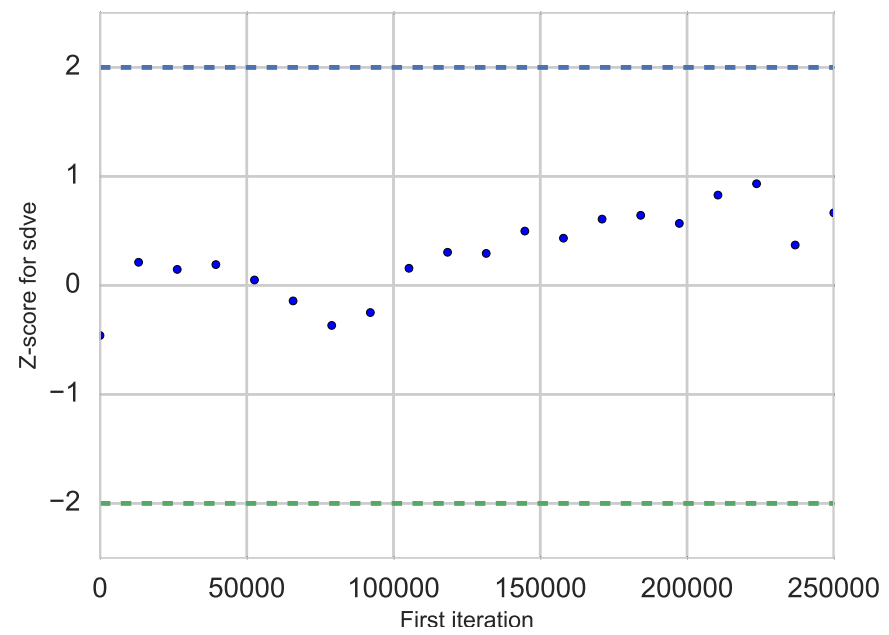

Figure 2.4: Geweke z-score for a variable. The scores fall within 2 standard deviations and convergence is reached

For updating the models presented in the previous section, three steps are followed. The application of the methodology follows the calibration and predictive model selection technique [57]. Under this approach, at least three experimental sets of data for the same experiment are needed. Figure 2.6 summarizes the methodology for updating a model. 


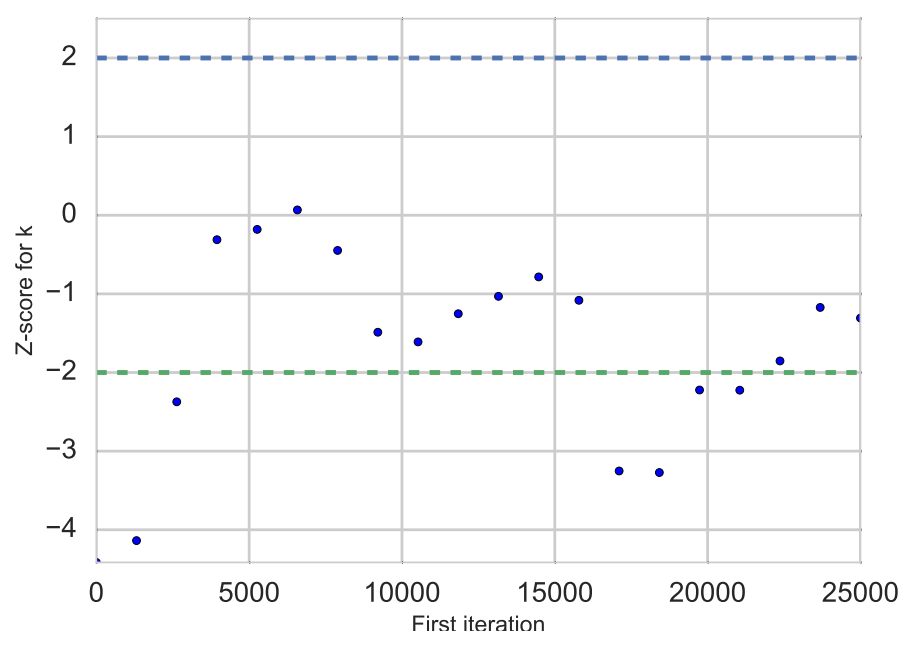

Figure 2.5: Geweke z-score for a variable. Some scores are out of the 2 standard deviations indicating a lack of convergence and additional samples are needed

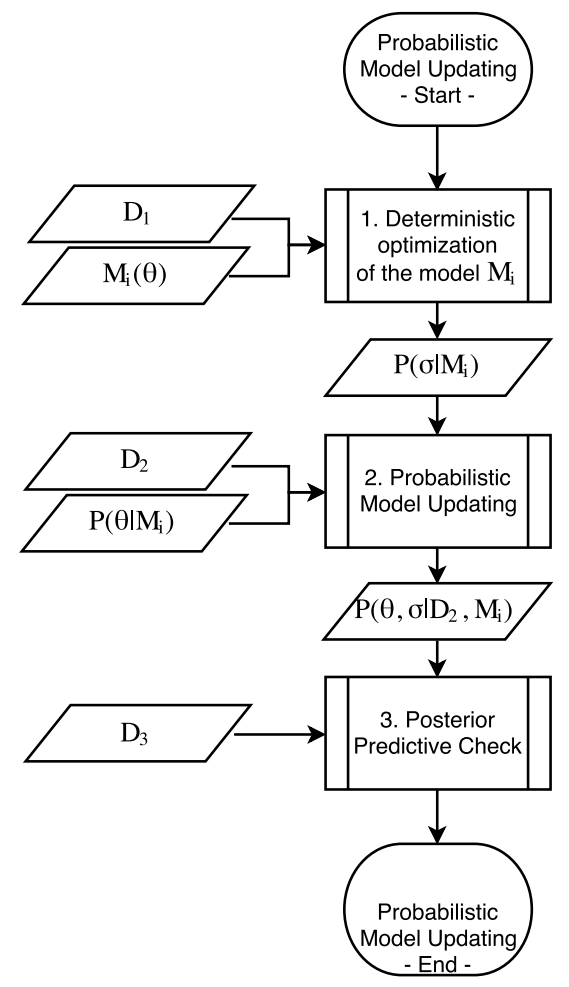

Figure 2.6: Probabilistic model updating flow chart

The first set of experimental data, $D_{1}$, is used for generating a preliminary optimization where the expected error and information about the parameters are found. The optimization is based on the minimization of the mean square error (MSE) be- 
tween the data, $D_{1}$ and the model, $M_{i}$. This minimization provides information about the expected error, therefore, it provides information for prior knowledge of the standard deviation $\sigma$ used in the likelihood estimation.

The second experimental dataset, $D_{2}$, is used for the calibration of the model based on the prior information obtained for the standard deviation of the likelihood $P\left(\sigma \mid M_{i}\right)$, and the prior knowledge of the parameters of the model $i, P\left(\theta \mid M_{i}\right)$. The results of this calibration can be used to estimate new data and to develop a posterior predictive check. This verifies the model comparing predictions with the observations of the third experimental dataset, $D_{3}$. In other words, the samples are used to model the phenomenon taking into account the uncertainty of the parameters and the uncertainty of the model. In this research, the prediction check is performed over the $95 \%$ credible interval.

The process for updating the HSI models variables is performed in two steps. First, the parameters of the structure $\left(\Theta_{s}=k_{s}, c_{s}, m_{s}\right)$ should be estimated using experimental data from the empty condition, that is, with no humans over the structure (Table 2.1). Then, the parameters of the system created by the interaction between the human (Table 2.2) and the structure (Table 2.1) are estimated using data from the occupied structure. Table 2.3 summarizes the models to be updated. Figure 2.7 shows the flow chart used for updating the human-structure interaction models.

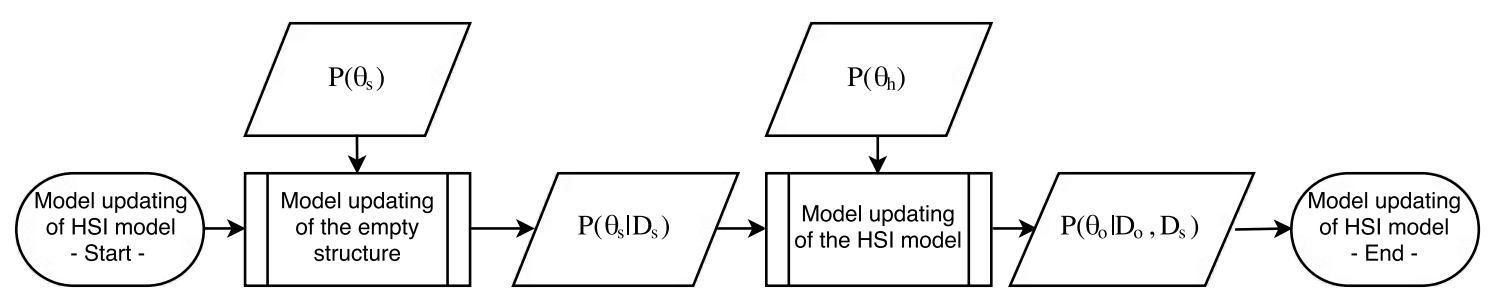

Figure 2.7: Flow chart for updating HSI models

The information gained in the first model for the empty condition is then used as 
Table 2.1: Models of the structure

\begin{tabular}{lc}
\hline Model & Variables \\
\hline CKM & $\theta_{s}=\left\{c_{s}, k_{s}, m_{s}\right\}$ \\
Poles and Zeros & $\theta_{s}=\left\{\omega_{1}, \omega_{2}, \zeta_{1}, \zeta_{2}, \omega_{z 1}, \zeta_{z 1}, K\right\}$ \\
\hline
\end{tabular}

Table 2.2: Models of the human

\begin{tabular}{lc}
\hline Model & Variables \\
\hline SDOF & $\theta_{h}=\left\{c_{h}, k_{h}, m_{h}\right\}$ \\
2DOF & $\theta_{h}=\left\{c_{h 1}, k_{h 1}, m_{h 1}, c_{h 2}, k_{h 2}, m_{h 2}\right\}$ \\
PD Controller & $\theta_{h}=\left\{K_{p}, t_{d}\right\}$ \\
PI Controller & $\theta_{h}=\left\{K_{p}, t_{i}\right\}$ \\
PID Controller & $\theta_{h}=\left\{K_{p}, t_{d}, t_{i}\right\}$ \\
\hline
\end{tabular}

Table 2.3: Models to be updated

\begin{tabular}{lcc}
\hline Model & Structural model & Human model \\
\hline 1 & CKM & PID controller \\
2 & CKM & PI controller \\
3 & CKM & PD controller \\
4 & CKM & SDOF \\
5 & CKM & 2DOF \\
\hline $6 \mathrm{~A}, 6 \mathrm{~B}, 6 \mathrm{C}$ & Poles and Zeros & Best Controller \\
\hline
\end{tabular}

prior information for the HSI models. Therefore, the parameters of the structure are updated using:

$$
P\left(\Theta_{s} \mid D_{s}\right) \propto P\left(D_{s} \mid \Theta_{s}\right) P\left(\Theta_{s}\right)
$$

The parameters of the structure and the HSI model are estimated using:

$$
P\left(\Theta_{o} \mid D_{e}, D_{o}\right) \propto P\left(D_{o} \mid \Theta_{o}\right) P\left(\Theta_{s} \mid D_{s}\right) P\left(\Theta_{h}\right)
$$

where $\Theta_{h}$ is the prior knowledge of the variables used for the human as shown in table 2.2. Notice that no assumption is made about the dependency between 
parameters of the structure $\left(\theta_{s}\right)$ and parameters of the human $\left(\theta_{h}\right)$. This assumption will be checked with the PDFs of the posterior once the model is updated.

The prior distributions for the structural parameters $\left(k_{s}, m_{s}\right.$, and $\left.c_{s}\right)$ are defined based on the physical characteristics of the structure.

\subsection{Probabilistic Model Selection}

Bayesian probabilistic model selection technique is used to determine which of the models proposed in the first 5 rows of Table 2.3 has the highest probability given the observed data. This methodology considers the uncertainty of the model and the uncertainty associated with each parameter. The probabilistic model selection is based on the principle proposed by William of Ockham, called Ockham's razor, which is well discussed in [58]. This principle states that "a simpler explanation for some phenomenon is more likely to be accurate than more complicated explanations" [59]. Therefore, models with a larger number of parameters are "penalized" to avoid overfitting.

Probabilistic model selection can be derived from the Bayes theorem shown in equation 2.9:

$$
P\left(\Theta \mid D, M_{j}\right)=\frac{P\left(D \mid \Theta, M_{j}\right) P\left(\Theta \mid M_{j}\right)}{P\left(D \mid M_{j}\right)}
$$

where the denominator, $P\left(D \mid M_{j}\right)$, is called model evidence and serves as a normalization constant. Therefore:

$$
p\left(D \mid M_{j}\right)=\int_{\Theta} p\left(D \mid \Theta, M_{j}\right) p\left(\Theta \mid M_{j}\right) d \Theta
$$

Using again Bayes inference, the posterior probability of a model, $M_{j}$, given some observations, $D$, can be estimated as: 


$$
P\left(M_{j} \mid D\right)=\frac{P\left(D \mid M_{j}\right) P\left(M_{j}\right)}{P(D)}
$$

If the same dataset, $D$, is used for updating models $M_{j}$ and $M_{k}$, the posterior odds ratio between the models is expressed by:

$$
\frac{P\left(M_{j} \mid D\right)}{P\left(M_{k} \mid D\right)}=\frac{P\left(D \mid M_{j}\right) P\left(M_{j}\right)}{P\left(D \mid M_{k}\right) P\left(M_{k}\right)}
$$

where the terms $P\left(D \mid M_{j}\right)$ and $P\left(D \mid M_{k}\right)$ are calculated following the equation 2.12. The terms $P\left(M_{k}\right)$ and $P\left(M_{j}\right)$ refer to the prior knowledge of each model. In this research, it is assumed that all the models have the same probability, therefore:

$$
K=\frac{P\left(M_{j} \mid D\right)}{P\left(M_{k} \mid D\right)}=\frac{P\left(D \mid M_{j}\right)}{P\left(D \mid M_{k}\right)}
$$

Where the term $K$ is called Bayes Factor. This research uses the widely known method of Monte Carlo (MC) integration [60] for obtaining the model evidence. The Vegas algorithm [61], implemented in the package Vegas, in the software Python, is used for MC integration.

Models are compared based on Kass and Raftery [1] which is a derivation of the Jeffreys' scale [62]. This scale classifies the Bayes factor as shown in Table 2.4 using the natural logarithm of the Bayes Factor, $(2 \operatorname{Ln}(k))$.

Table 2.4: Kass and Raftery scale for Bayes factor

\begin{tabular}{cc}
\hline $2 \mathrm{Ln}(\mathrm{K})$ & Strength of evidence \\
\hline 0 to 2 & not worth more than a bare mention \\
2 to 6 & Positive \\
6 to 10 & Strong \\
$>10$ & Very strong \\
\hline
\end{tabular}




\section{Chapter 3 \\ EXPERIMENTAL TESTING AND UPDATING OF EMPTY STRUCTURE}

The updating of the human-structure interaction models is performed using experimental tests over a configurable structure. This chapter focuses on the description of the structure, the equipments used, the tests performed, and the model update process implemented for updating the parameters of the unoccupied structure.

\subsection{LAB StRUCTURE}

The structure used in testing is a steel frame specifically designed to represent a range of dynamic properties representative of flexible slabs; however, a "rigid" behavior $\left(f_{n} \approx 10 H z\right)$ can also be reached. The experimental setup is light in order to maintain the ratio between live load and dead load similar to common structures susceptible to human-induced vibrations. The structure is inspired by an existing experimental setup at Bucknell University [18].

The frame is a cantilever horizontal truss composed by $5 \mathrm{x} 4 \mathrm{x}^{1 / 4^{\prime \prime}}$ steel tubes, built in the Structures lab at the University of South Carolina as shown in Figure 3.1. The structure has four supports. Two supports are fixed and located at one end. The other two can move along the structure in order to modify the cantilever length, which change the dynamic properties of the structure. Additional concrete blocks are used to customize the mass. These are heavier than the steel frame and lead to a better control of the desirable conditions. Changes in the stiffness and mass lead to 
modify its dynamics from rigid $\left(f_{n} \approx 10 H z\right)$ to flexible $\left(f_{n} \approx 3.0 H z\right)$.

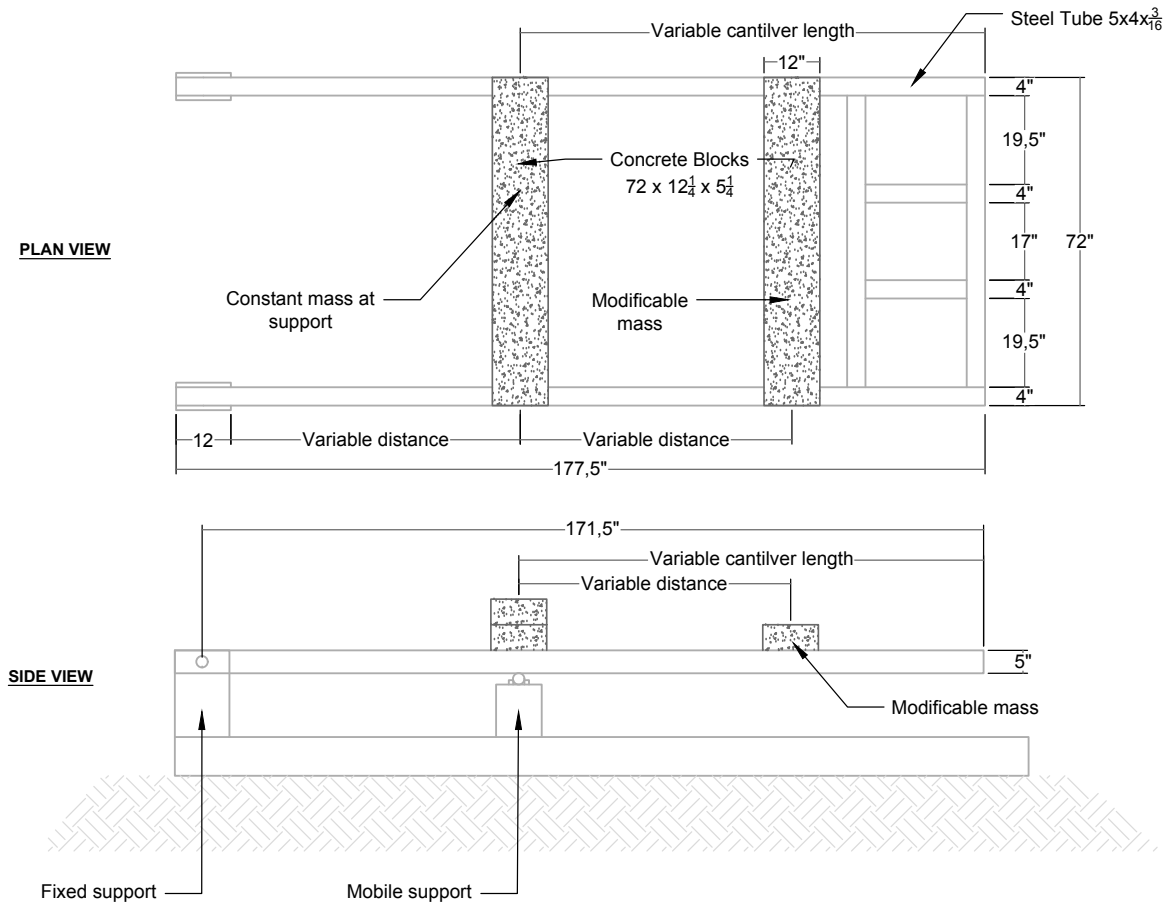

Figure 3.1: Plant and side views of lab specimen

Figure 3.2 shows one structure's configurations used during the tests. The cantilever mass is provided by the two concrete blocks at the end (to the left). The picture also shows the fixed support, which is on the right end, and the mobile support in the middle.

\subsection{InstRUMENTATION AND TESTS}

Impact hammer tests were used for the estimation of the transfer function of the structure. The structure was equipped with one PCB 333B50 accelerometer with a sensitivity of $100 \mathrm{mV} / \mathrm{g}$. The vibration was induced by an impact hammer, PCB 096D50, with a sensitivity of $0.2305 \mathrm{mV} / \mathrm{N}$. The hammer can record a maximum force 


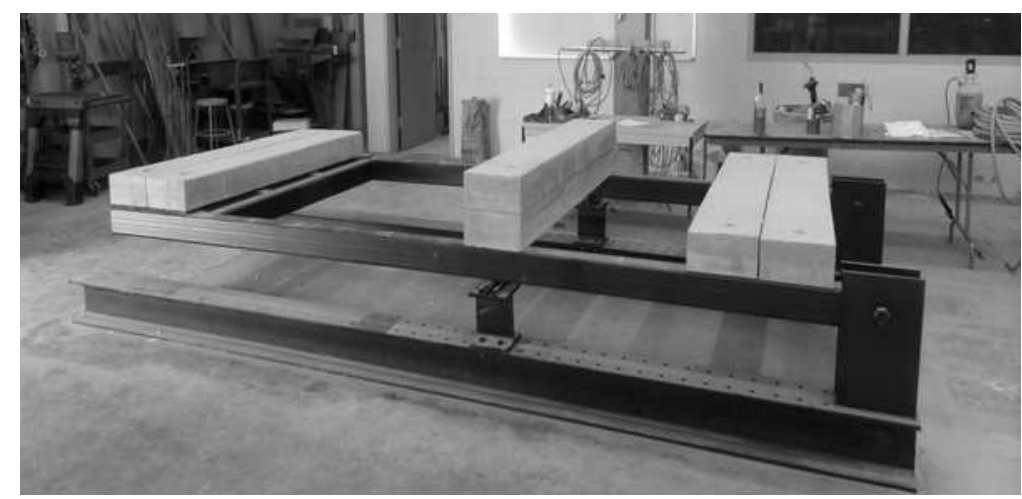

Figure 3.2: Test configurations: Empty structure

of $\pm 22240 \mathrm{~N}$. The accelerometer was placed in a vertical direction in the middle of the span. The impact was induced over the concrete blocks in the cantilever, in the middle of the span, at a distance of less than $10 \mathrm{~cm}$ from the border of the concrete slab. Figure 3.3 shows a picture at the moment of hitting the structure.

The data acquisition system consists of a modular NI CompactDAQ with a NI9234 module. Data was acquired using a sampling frequency of $1652 \mathrm{~Hz}$, then resampled to $150 \mathrm{~Hz}$. The duration of each experimental record was 20 seconds, starting 4 seconds before the impact. Three tests with the empty structure were used for the parameter updating of each configuration. Other configurations of the structure involve similar equipment and tests. Figure 3.4 shows the typical records for the acceleration response and the input force applied to the structure.

The estimation of the transfer function is performed using [63]:

$$
\hat{T F}=\frac{P_{x y}(f)}{P_{x x}(f)}
$$

where $P_{x y}$ is the cross power spectral density between the acceleration of the structure and the force of the hammer, and $P_{x x}$ is the auto power spectral density of the force of the hammer. Transfer function of the experimental data are shown in Figures 3.5 and 3.8, during the description of each structure's configuration. 


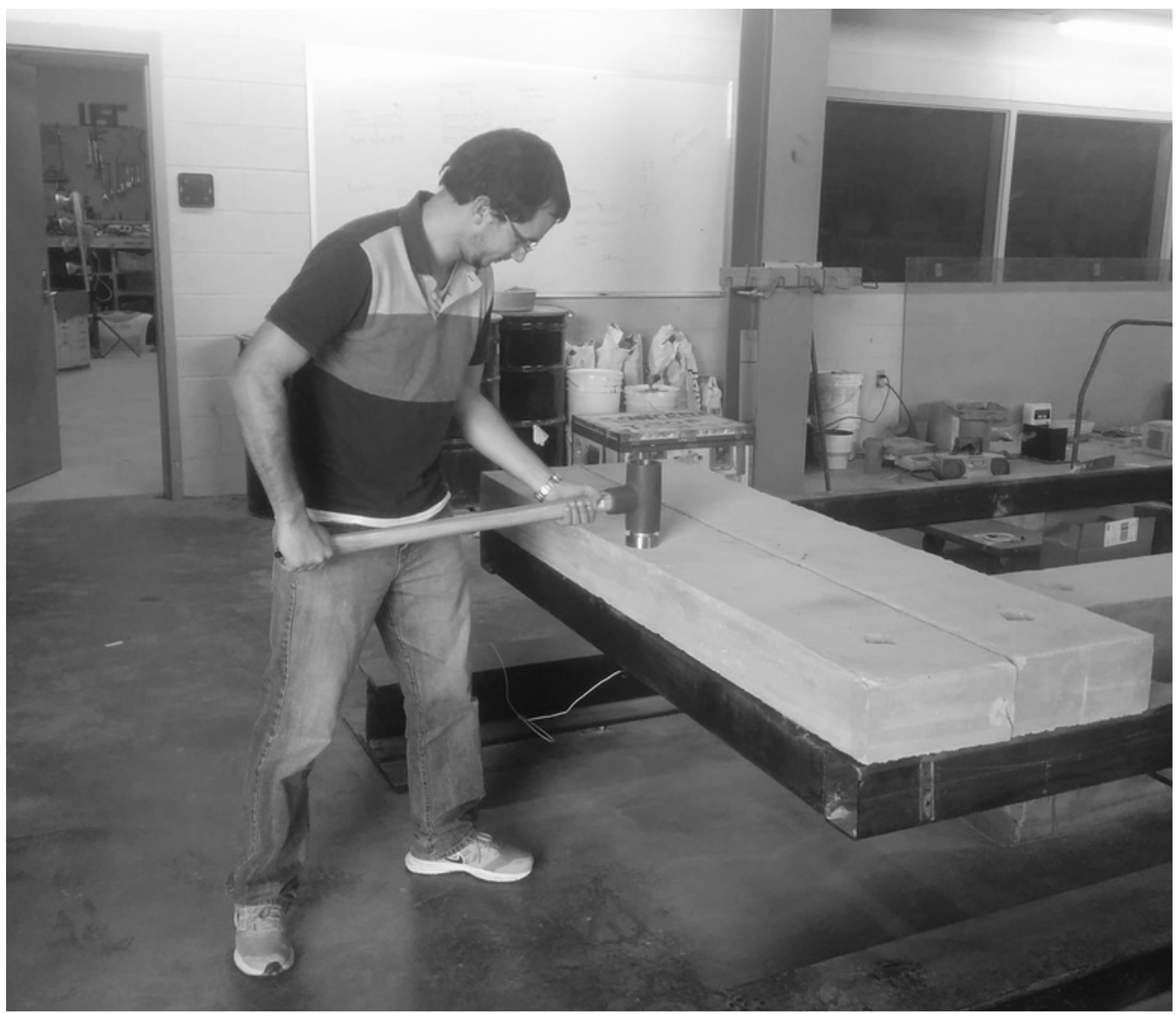

Figure 3.3: Typical free vibration test performed at the structure
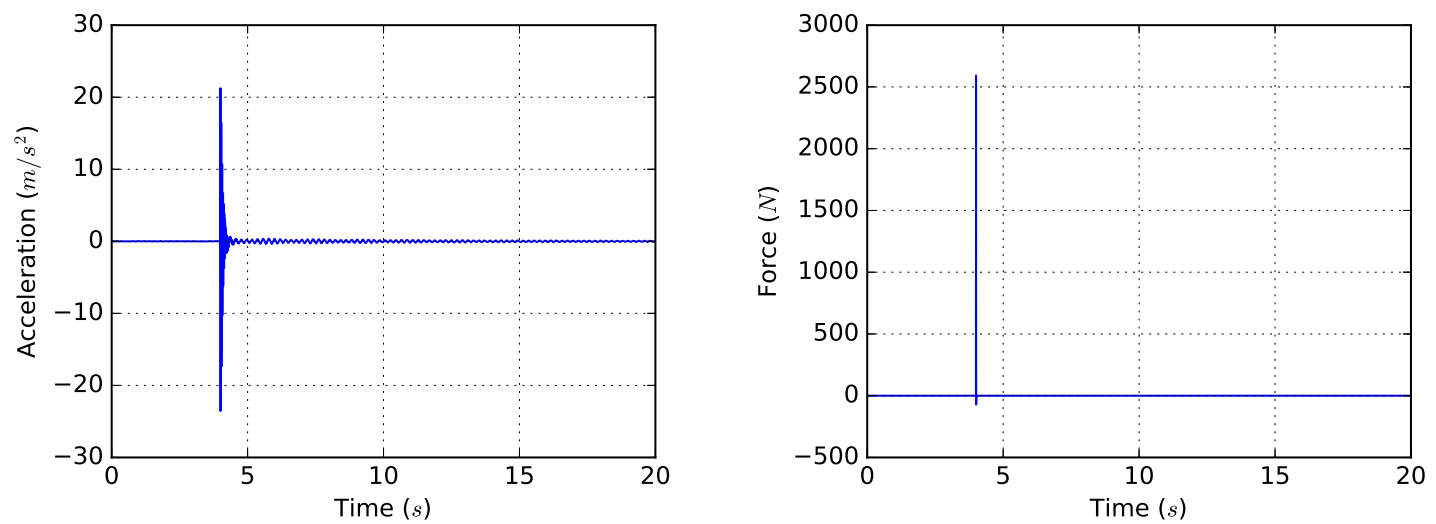

Figure 3.4: Typical acceleration (left) and force (right) data recorded from the impact tests over the structure 


\subsection{Parameters of the StruCture}

Obtaining the parameters of the structure is a crucial task before developing the controller model. Two different modeling approaches were used to identify the structure as mentioned in the previous chapter. The selection of the model approach depends on the configuration and the experimental modal information observed in the transfer function. Based on the configuration, one vertical mode or two (one vertical and horizontal) modes are observed in the interest range of frequencies. The participation of a horizontal vibration mode in a vertical vibration test is the product of the interaction of two factors: the mode is not totally vertical, and a small component of the force is horizontal.

When a predominant peak is observed in the transfer function, the influence of a lateral vibration mode is not modeled, and the structure can be modeled as a single degree of freedom system with three parameters: $m_{s}, c_{s}, k_{s}$. In the case that an additional horizontal mode is identified in the transfer function, the model of the structure uses the poles and zeros approach described in section 2.1.

\section{Configuration A}

The first configuration (called Configuration $A$ ) is used to evaluate the humanstructure interaction when a single occupant is over the structure. For this configuration, the cantilever length is 80in and the concrete blocks are located 66.4in from the support. The full experimental transfer function of the structure is shown in Figure 3.5. A single predominant peak is observed, therefore the model will be updated using a single degree of freedom model with parameters $\Theta_{s}=\left\{m_{s}, c_{s}, k_{s}\right\}$. The likelihood is estimated using the $n=25$ points closer to the peak on the transfer function as also shown in Figure 3.5. This corresponds to the values of the transfer function between $28 \mathrm{rad} / \mathrm{s}$ and $38 \mathrm{rad} / \mathrm{s}$. Points selected around the peak were chosen 
because this is the range of frequencies that will cause significant vibration problems (around resonance).

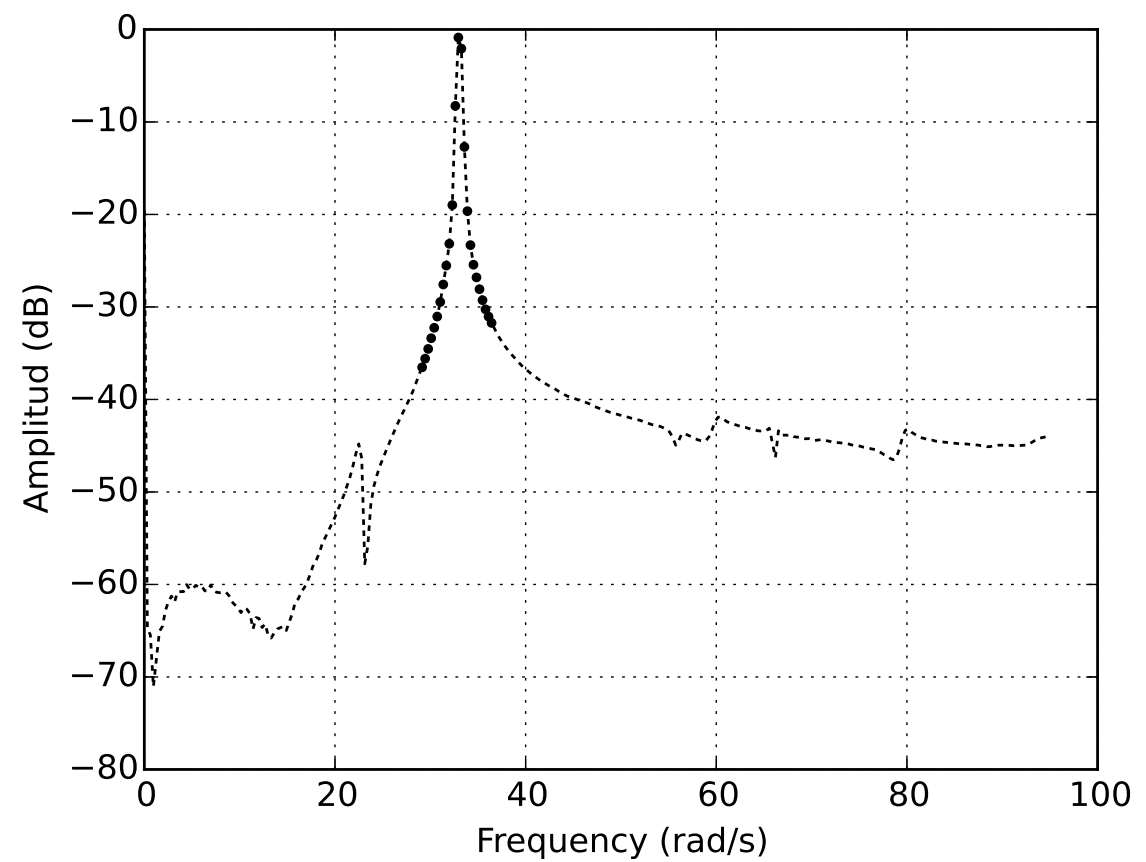

Figure 3.5: Experimental transfer function of the empty structure and data (bold dots) used for updating the configuration A

The prior distributions for the structural parameters $\left(k_{s}, m_{s}\right.$, and $\left.c_{s}\right)$ are defined based on the measurements of the structure. The mass of the structure, $m_{s}$, is assumed to follow a normal distribution. The mean of $m_{s}$ is obtained based on the sum of the concrete block masses and the cantilever mass of the frame as described in the equation 3.2. The standard deviation is assumed $10 \%$ of the mean value.

$$
\bar{m}_{s}=m_{b l}+m_{e f}
$$

The term $m_{b l}$ refers to the lumped mass of the concrete blocks in the cantilever. The approximate mass of one concrete block is $178 \mathrm{~kg}$. The effective mass of the first vibration mode of the frame, $m_{e f}$, is calculated based on the total truss mass in the cantilever, $m_{t c}$, using the ratio $m_{e f}=0.2357 m_{t c}$, from Chopra [64]. The prior PDF 
distribution of the stiffness of the structure, $k_{s}$, is assumed uniform between a range of $300 \mathrm{kN} / \mathrm{m}$ and $500 \mathrm{kN} / \mathrm{m}$.

The prior information for the damping coefficient, $c_{s}$, is estimated based on a free vibration test performed in the structure. A damping ratio of $\zeta=0.2 \%$ is estimated using the Free Decay Motion technique such as explained in Chopra [64]. Therefore, the prior $\mathrm{PDF}$ for $c_{s}$ is $P\left(c_{s}\right)=N(31,3.1)$.

The prior distribution of the standard deviation of the likelihood consist of an Inverse Gamma distribution where the shape parameter, $\alpha$, is 10 , and the scale parameter, $\beta$, is obtained from the minimization of the Mean squared error (MSE) calculated between the SDOF model and the first set of experimental data. The minimization uses the Nelder-Mead method [65]. Table 3.1 shows the prior used for the calibration of the model.

Table 3.1: Prior PDFs used for updating the parameters

\begin{tabular}{cccc}
\hline Parameter & units & PDF & Parameters \\
\hline$c_{s}$ & $N s / m$ & Normal & $\mu=31.0, \sigma=3.10$ \\
$k_{s}$ & $k N / m$ & Uniform & lower $=300.0$, upper $=500.00$ \\
$m_{s}$ & $k g$ & Normal & $\mu=377.0, \sigma=16.00$ \\
$\sigma_{s}$ & & Inverse Gamma & $\alpha=10.0, \beta=3.07$ \\
\hline
\end{tabular}

The posterior PDF $\left(P\left(\Theta_{s} \mid D_{s}\right)\right)$ was estimated using Gibbs sampling [66]. A total of 1 millon of samples were obtained. However, 500,000 samples were used, and others 500,000 were used for thinning. The plots in the diagonal of Figure 3.6 show the marginal histograms for each variable. The second plot in the second row shows the values of $m_{s}$ and $k_{s}$ for all the samples. The plot indicates a strong correlation between the stiffness and the mass, which is expected since the natural frequency of the $\mathrm{SDOF}$ is a function of $\frac{k}{m}$. The model updating procedure estimated this correlation even though the priors do not make any assumptions about the dependency between parameters. These two variables could have been reduced to another variable, $\beta=\frac{k}{m}$. However, it was kept as separate variables for the rest of the analysis as a way to verify 

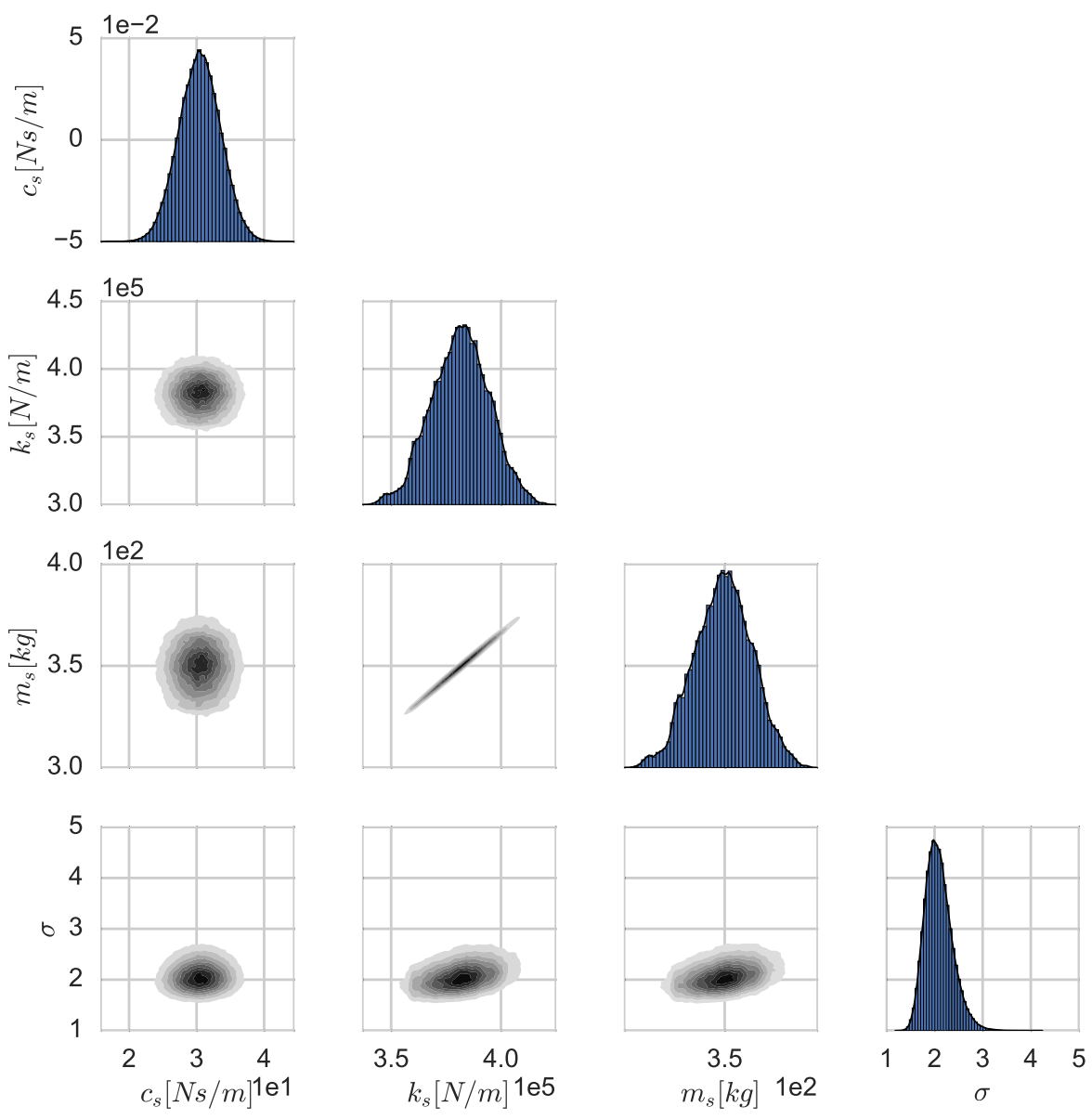

Figure 3.6: Marginal histograms and samples generated to updated the SDOF model of the Configuration A

that the model updating technique was correctly applied. In other words, the $k_{s}$ and $m_{s}$ parameters should still be correlated once the person steps on the structure and the combined HSI model is updated. Figure 3.6 also shows no correlations between the damping to the mass and the stiffness. These results are not surprising because damping is, in general, a parameter difficult to identify [67].

The transfer function of the experimental data and the analytical transfer function built with the samples of the parameters are shown in Figure 3.7. 


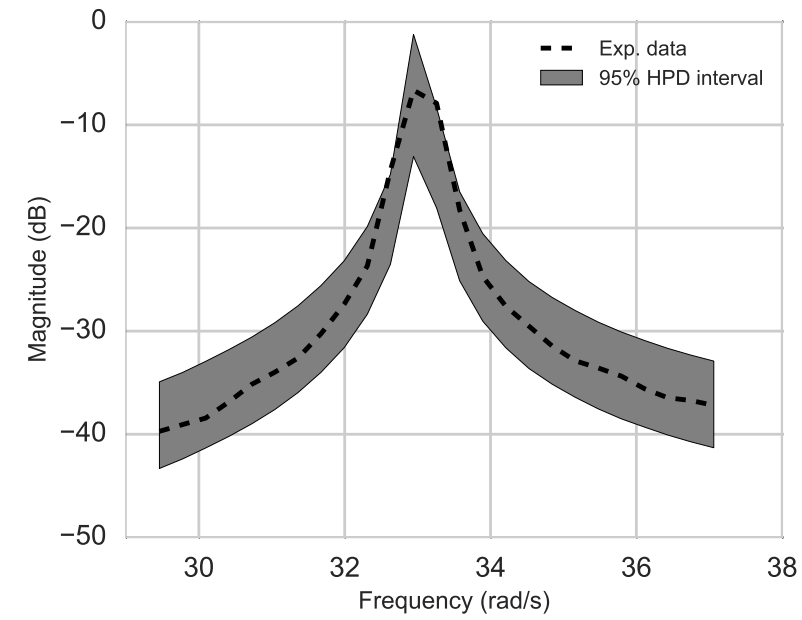

Figure 3.7: Posterior predictive check of the Configuration A

\section{Configuration B}

The second structure configuration (called Configuration B) is used to evaluate the human-structure interaction when groups of two and three occupants are over the structure. For this configuration, the cantilever length is 100in and the concrete blocks are located $75 \mathrm{in}$ from the support. The full experimental transfer function of the structure is shown in Figure 3.8. Two close predominant peaks are observed, therefore the model is updated using a poles and zeros model. The likelihood is estimated using the $n=100$ points closer to the higher peak on the transfer function, as also shown in Figure 3.8. This corresponds to the values of the transfer function between $17 \mathrm{rad} / \mathrm{s}$ and $32 \mathrm{rad} / \mathrm{s}$.

The prior distributions for the parameters $\left(\omega_{1}, \omega_{2}, \omega_{z 1}, \zeta_{1}, \zeta_{2}, \zeta_{z 1}, K\right)$ are defined based on direct measurements of the transfer function of the structure. The prior distribution of each frequency $\left(\omega_{1}, \omega_{2}, \omega_{z 1}\right)$ is obtained from the experimental transfer function using the peak-peaking method [68]. The priors for damping ratios $\left(\zeta_{1}, \zeta_{2}\right.$, $\left.\zeta_{z 1}\right)$ are obtained using uniform distributions over low damping ratios, based on the experimental tests, and from the previous configuration A. 


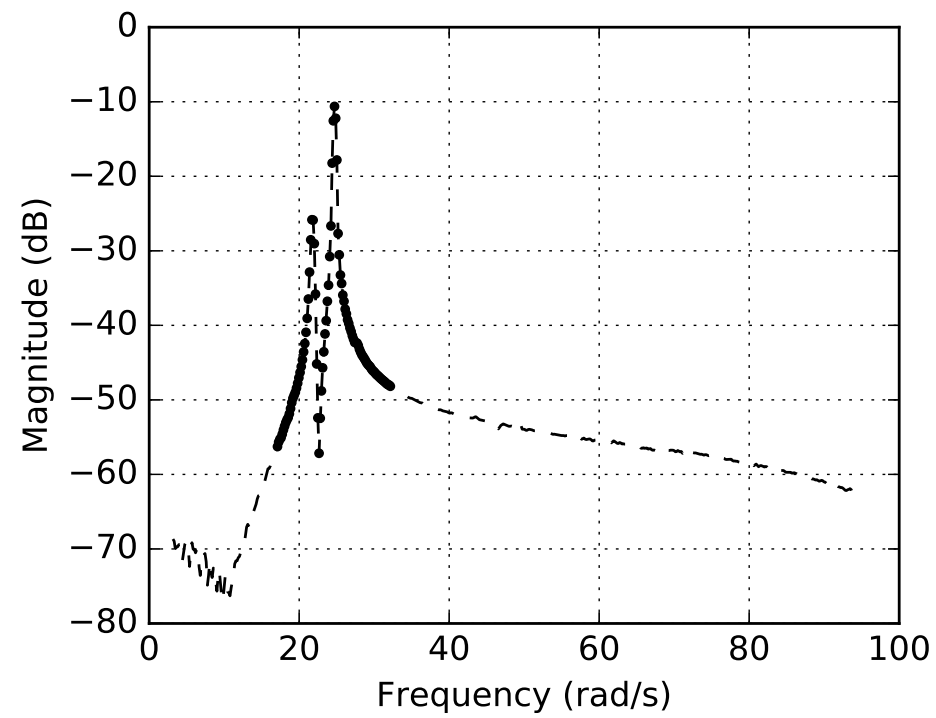

Figure 3.8: Experimental transfer function of the empty structure and data (bold dots) used for updating the configuration B

The prior distribution of the standard deviation of the likelihood, $\sigma_{l}$, is an Inverse Gamma obtained following the same procedure used in the SDOF model. The shape parameter, $\alpha$, is 10 , and the scale parameters, $\beta$, is obtained from the minimization of the Mean squared error (MSE). Table 3.2 summarizes the priors used for the calibration of the model.

Table 3.2: Prior PDFs used for updating the parameters

\begin{tabular}{cccc}
\hline Parameter & units & PDF & Parameters \\
\hline$\omega_{1}$ & $\mathrm{rad} / \mathrm{s}$ & Normal & $\mu=21.8, \sigma=0.22$ \\
$\omega_{2}$ & $\mathrm{rad} / \mathrm{s}$ & Normal & $\mu=24.7, \sigma=0.25$ \\
$\zeta_{1}$ & {$[\%]$} & Uniform & lower $=0.001$, upper $=0.90$ \\
$\zeta_{2}$ & {$[\%]$} & Uniform & lower $=0.001$, upper $=0.90$ \\
$\omega_{z 1}$ & $\mathrm{rad} / \mathrm{s}$ & Normal & $\mu=22.7, \sigma=0.23$ \\
$\zeta_{z 1}$ & {$[\%]$} & Uniform & lower $=0.001$, upper $=0.90$ \\
$K$ & & Normal & $\mu=0.0, \sigma=0.5$ \\
$\sigma_{l}$ & & Inverse Gamma & $\alpha=10.0, \beta=3.07$ \\
\hline
\end{tabular}

The number of samples for obtaining convergence of the samples are 300,000. Figures 3.9, 3.10 and 3.11 show the marginal histograms for each variable (diagonal) 

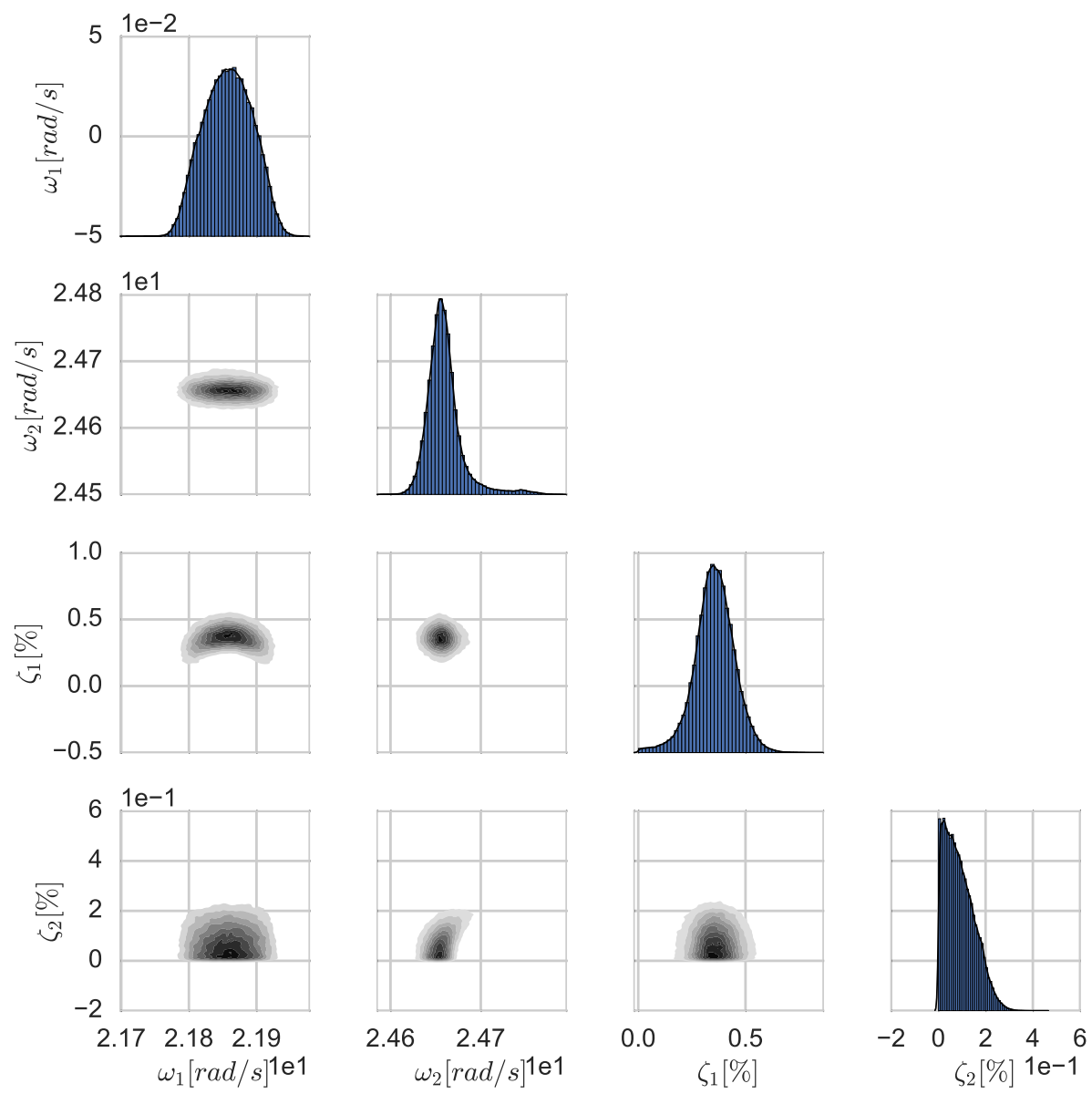

Figure 3.9: Marginal histograms and samples generated to update the Two DOF model of the Configuration B (Part 1 of 3 )

and the samples correlation. No correlation between the parameters is observed, further than the frequencies and damping of each pole. The posterior predictive check of the updated model was compared with an unused experimental dataset. Figure 3.12. shows the 95\% HPD interval of the model is compared to the third experimental dataset. 

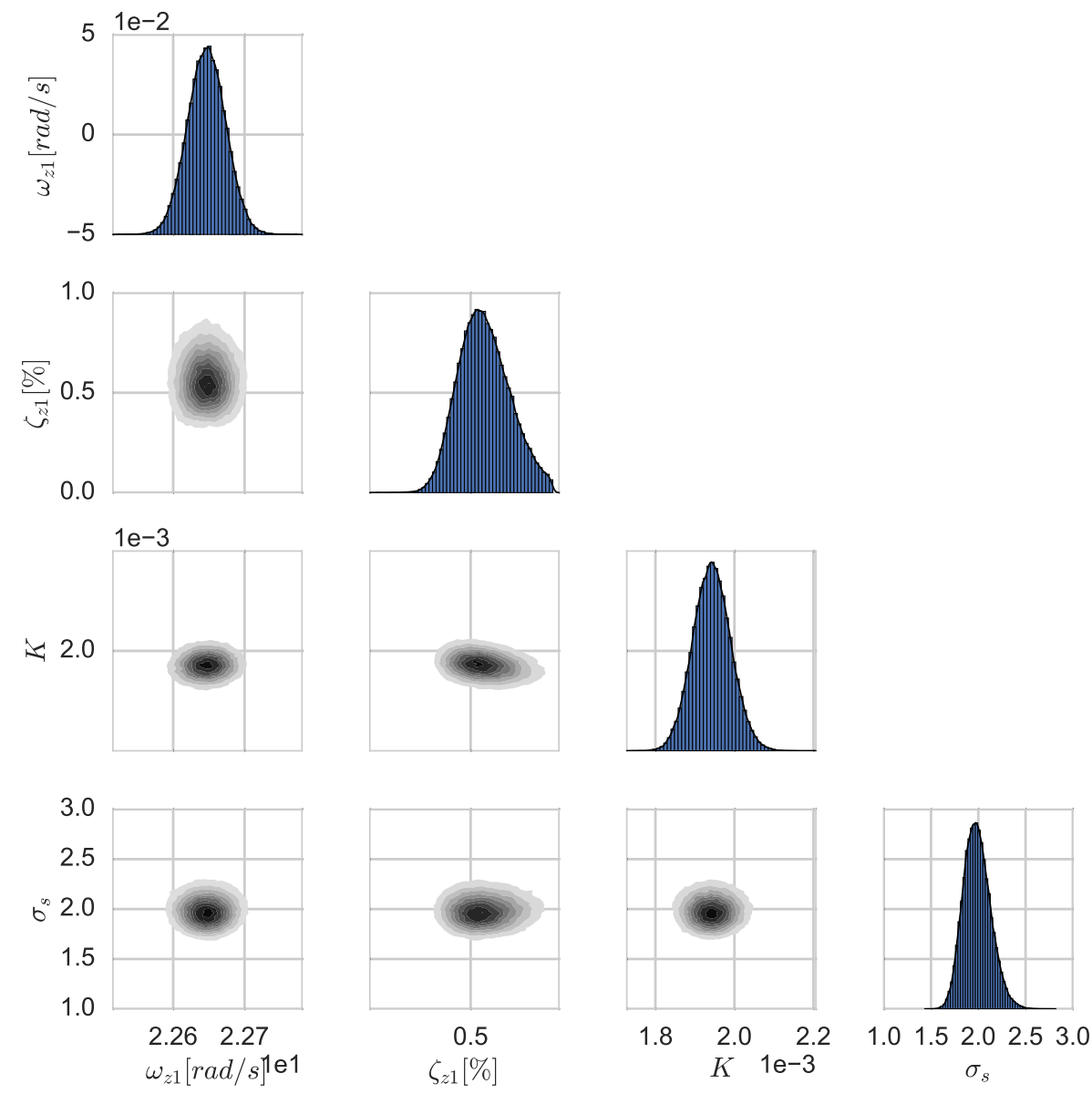

Figure 3.10: Marginal histograms and samples generated to update the Two DOF model of the Configuration B (Part 2 of 3) 

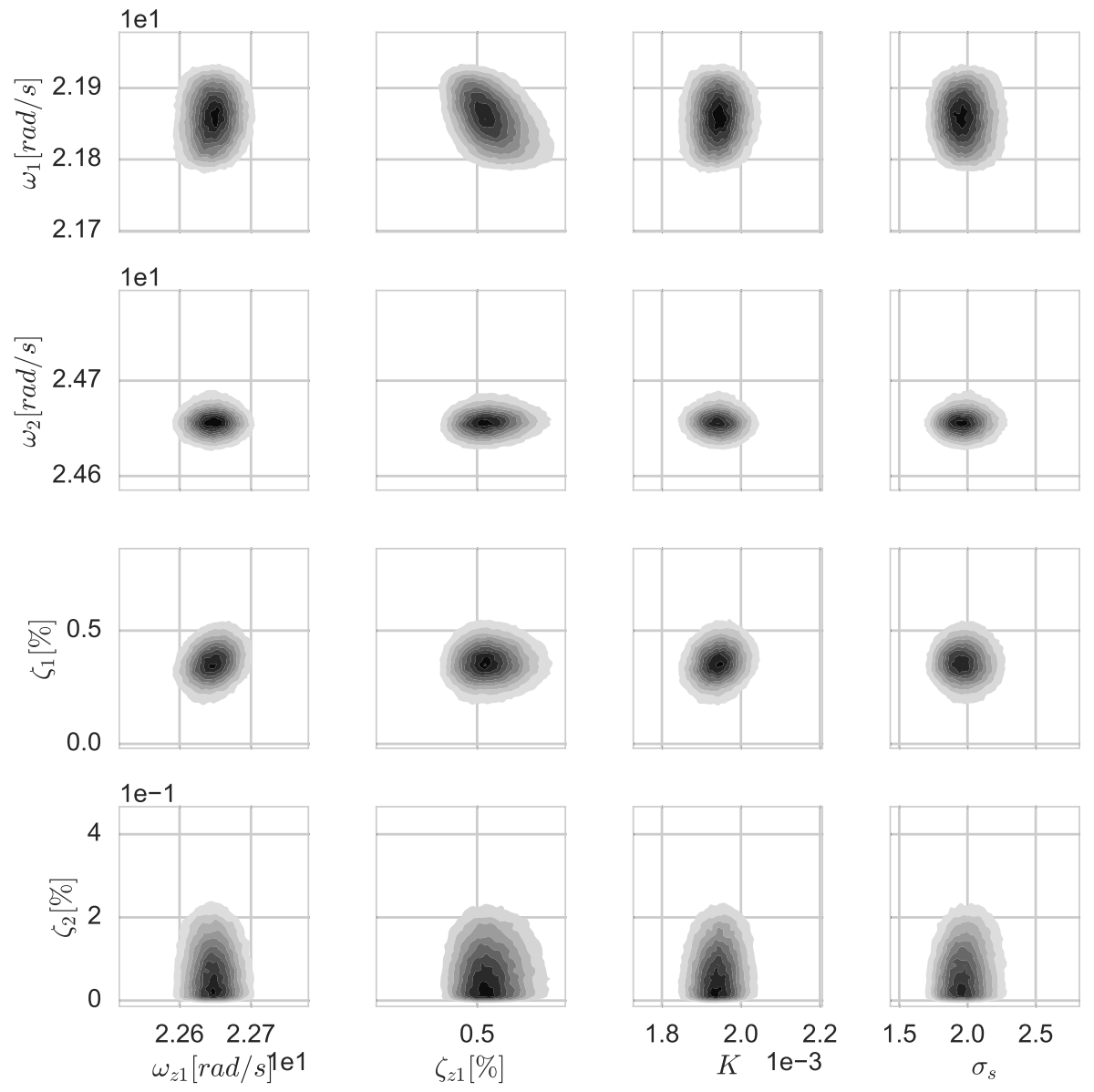

Figure 3.11: Marginal histograms and samples generated to update the Two DOF model of the Configuration B (Part 3 of 3)

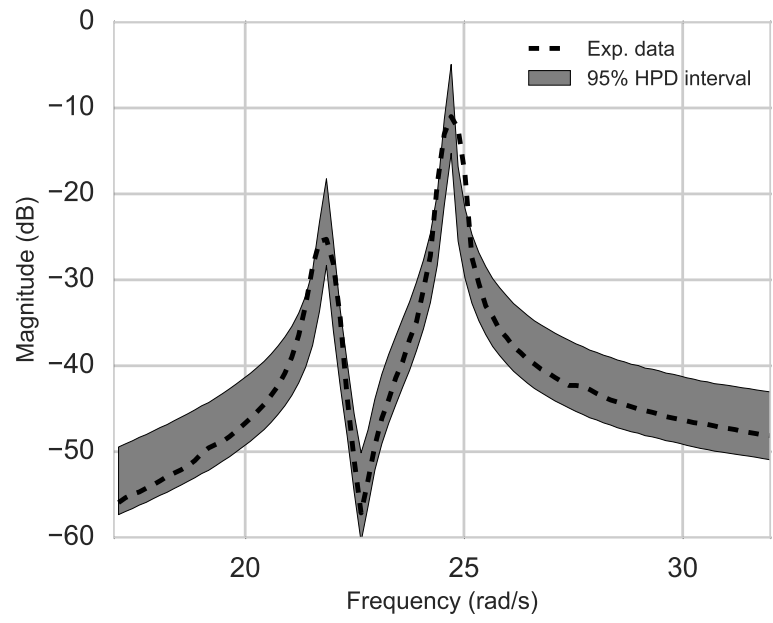

Figure 3.12: Posterior predictive check of the Configuration B 


\subsection{CONCLUSion REMARKS}

In this chapter, two models of the empty structure were updated based on the transfer function. Experimental data were obtained using impact hammer tests for inducing free vibration of the structure. The transfer function of the experimental data was used for the identification of the parameters of the models using Bayes inference. The obtained posterior distributions of the parameters of each model will be used as a prior information of the models updated in the next section (HSI models). The models updated showed good fit to unused experimental data. The correlations of the parameters $k_{s}$ and $m_{s}$ for the SDOF model, and $\omega_{1}-\zeta_{1}$ and $\omega_{2}-\zeta_{2}$ for the Two DOF model result similar to the dependencies found in the literature, which were not included in the priors. 


\section{CHAPTER 4}

\section{RESUlts}

The model updating and comparison results for models for single individuals over the structure discussed in Chapter 2 are shown in this section. The models are updated for single individuals. Models are validated by changing the dynamic characteristics of the structure and using the model of the person to predict the behavior of the overall human-structural system. An evaluation of each model is performed using the model selection technique detailed previously in Section 2.3.

Figure 4.1 shows the transfer functions for the empty and occupied conditions obtained from the experiments of Configuration A. As expected, the occupied structure has a lower natural frequency than the empty structure. In addition, the damping ratio is higher when a person stands on the slab, which is unexpected if the person was only adding mass to the structure. This behavior reflects the observations given by other researchers reported in the literature $[9,69,7]$.

\subsection{Parameter Updating of The MOdels}

The parameters of human-structure interaction models are updated using the methodology discussed in Chapter 2. The parameters of the structure, $\theta_{s}=\{m, c, k\}$, are also updated in this process, however, their priors are the posteriors presented in Section 3.3. Therefore, sampling is performed for the joint distribution of all the parameters $\theta_{s}: \theta=\left[\theta_{s}, \theta_{h}\right]$. The number of total samples obtained from the Gibbs sampler to reach convergence is different for each model. Geweke z-score plots [56] are shown in Appendix B indicating the convergence of each chain. 


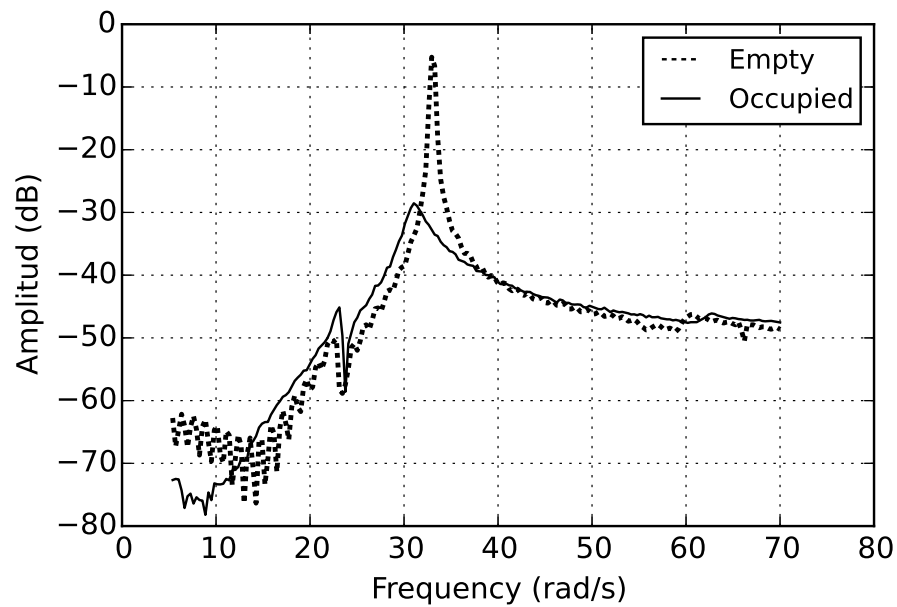

Figure 4.1: Empty and occupied transfer functions of the structure configuration A

The next section discusses the results for each of the models describe in Table 2.3 and repeated here for convenience.

Table 4.1: Models to be updated

\begin{tabular}{lcc}
\hline Model & Structural model & Human model \\
\hline 1 & CKM & PID controller \\
2 & CKM & PI controller \\
3 & CKM & PD controller \\
4 & CKM & SDOF \\
5 & CKM & 2DOF \\
\hline $6 \mathrm{~A}, 6 \mathrm{~B}, 6 \mathrm{C}$ & Poles and Zeros & Best Controller \\
\hline
\end{tabular}

\subsubsection{Models 1, 2, And 3 (Controller models)}

This section presents the model updating process for updating the model 1, 2, and 3. These models use the mass, stiffness, and damping to model the structure, and to model the human with a controller PID, PI or PD. The prior distributions of the structure are summarized in Table 4.2. The priors of the controllers are presented in Table 4.3. Stability conditions (Appendix A) are enforced such that the probability of the model is zero if the model is unstable. 
Table 4.2: Prior PDFs of the structure's parameters

\begin{tabular}{cccc}
\hline Parameter & units & PDF & Parameters \\
\hline$c_{s}$ & $N s / m$ & Normal & $\mu=28.9, \sigma=4.9$ \\
$k_{s}$ & $k N / m$ & Normal & $\mu=383245.2, \sigma=13438.2$ \\
$m_{s}$ & $k g$ & Normal & $\mu=350.9, \sigma=12.3$ \\
$\sigma_{s}$ & & Normal & $\mu=2.3, \sigma=0.3$ \\
\hline
\end{tabular}

Table 4.3: Prior definition for parameters of the PID, PI, and PD controller

\begin{tabular}{cccc}
\hline Parameter & Model & Distribution & Values \\
\hline$K_{p}$ & $1,2,3$ & Uniform & $\min =-10 E 3, \max =10 E 3$ \\
$T_{d}$ & 1,3 & Uniform & $\min =-10 E 3, \max =10 E 3$ \\
$T_{i}$ & 1,2 & Uniform & $\min =-10 E 3, \max =10 E 3$ \\
\hline
\end{tabular}

For all models, a total of 500,000 samples were used from the Gibbs sampler in order to reach convergence, from a total of 1 millon samples generated (500,000 burnin samples). The first points of the chains were the best values reported from the deterministic optimization of the model. Geweke plots are shown in Appendix B. Table 4.4 summarizes the number of samples and the location of the Geweke z-score plots in Appendix B.

Table 4.4: Number of samples used and page location of convergence plots

\begin{tabular}{lccc}
\hline Model & Samples & Burn-in & Geweke z-score \\
\hline 1 & 500,000 & 500,000 & pages $77-81$ \\
2 & 500,000 & 500,000 & pages $81-84$ \\
3 & 500,000 & 500,000 & pages $85-88$ \\
\hline
\end{tabular}

Figures 4.2, 4.3, 4.4 show the samples for the controllers' parameters. Figure 4.2 shows a high correlation between $t_{i}$ and $t_{d}$. This is expected because the stability conditions were established as additional priors to the model. This means that the human-structure system will be unstable for a set of $t_{i}$ and $t_{d}$ values is chosen out of this region. Within this correlation, one of the parameters can be modeled as a function of the other. Figure 4.3 shows some correlation between the parameters $k_{p}$ and $t_{i}$, however this is not related to the stability conditions of the system implemented 
as additional priors.
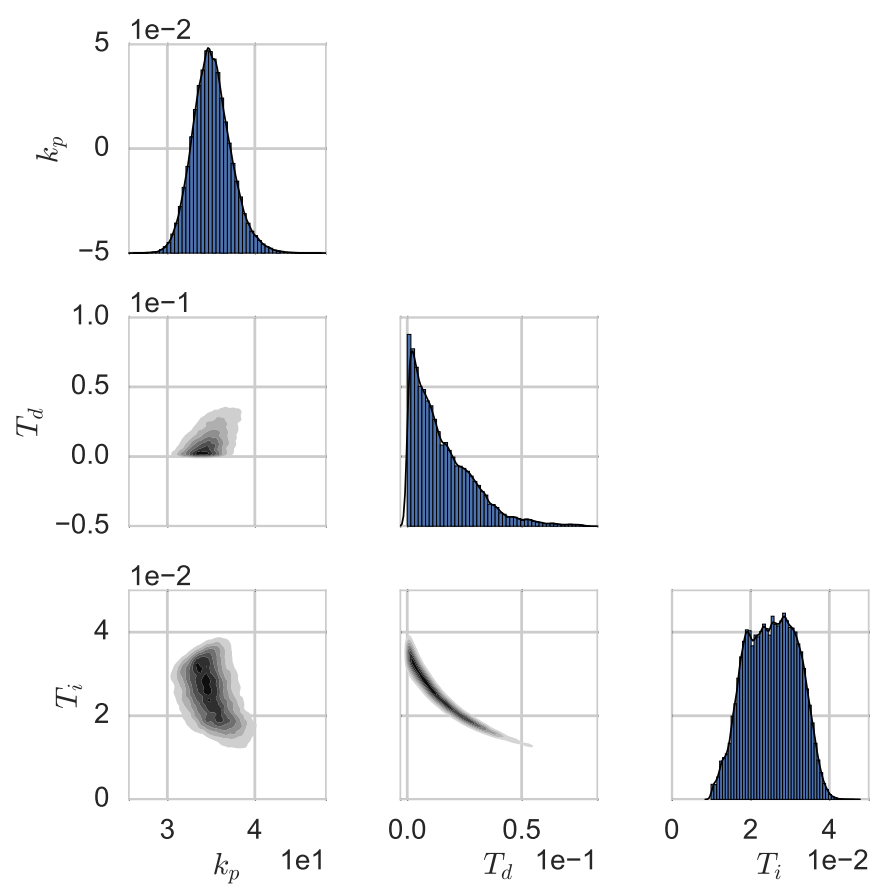

Figure 4.2: Marginal histogram (diagonal) and samples for PID controller parameters

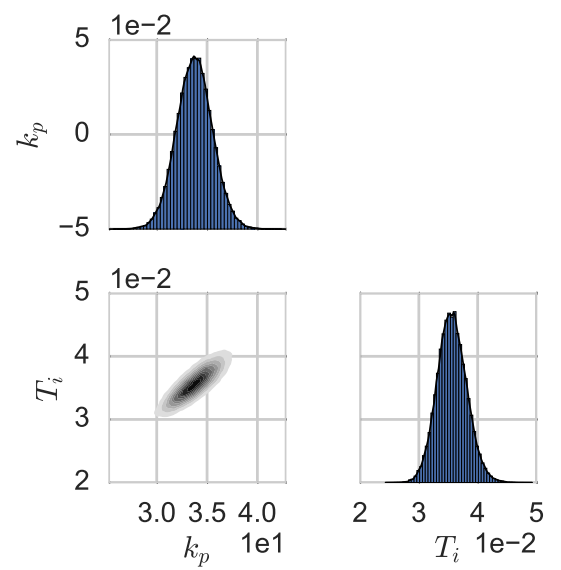

Figure 4.3: Marginal histogram (diagonal) and samples for PI controller parameters

The mean, standard deviation, and the 95\% High Probability Density (HPD) interval of the samples for each model are shown in Table 4.5. 


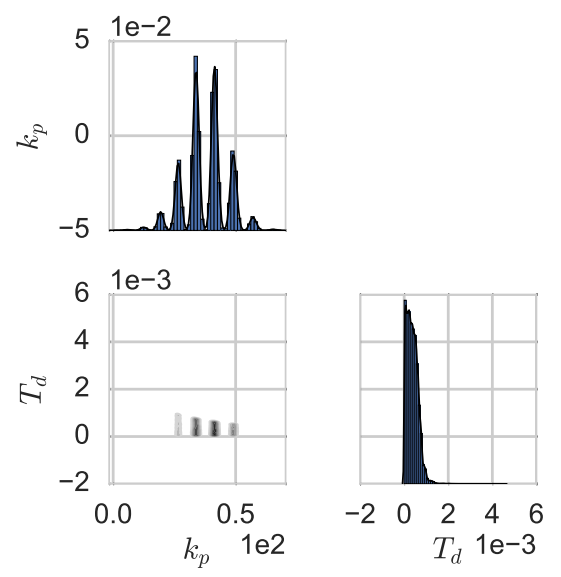

Figure 4.4: Marginal histogram (diagonal) and samples for PD controller parameters

Table 4.5: Moments of variables describing the parameters of models 1, 2 and 3

\begin{tabular}{|c|c|c|c|c|c|c|c|}
\hline Model & Parameter & $\begin{array}{c}c \\
{\left[\frac{N-s}{m}\right]}\end{array}$ & $\begin{array}{c}k \\
{[N / m]}\end{array}$ & $\begin{array}{c}m \\
{[k g]}\end{array}$ & $K_{p}$ & $t_{d}$ & $t_{i}$ \\
\hline \multirow{3}{*}{1} & Mean & 28.9 & $383 \mathrm{E} 3$ & 350.8 & 35.1 & 0.015 & 0.025 \\
\hline & STD & 0.5 & 1009 & 0.9 & 2.2 & 0.013 & 0.006 \\
\hline & $95 \% \mathrm{HPD}$ & $\begin{array}{l}(27.9, \\
29.9)\end{array}$ & $\begin{array}{l}(381 \mathrm{E} 3, \\
385 \mathrm{E} 3)\end{array}$ & $\begin{array}{l}(348.9, \\
352.5)\end{array}$ & $\begin{array}{l}(30.9, \\
39.5)\end{array}$ & $\begin{array}{c}(0 ., \\
0.041)\end{array}$ & $\begin{array}{l}(0.014, \\
0.037)\end{array}$ \\
\hline \multirow{3}{*}{2} & Mean & 28.9 & 383E3 & 350.8 & 33.7 & & 0.036 \\
\hline & STD & 0.5 & 1008 & 0.9 & 1.8 & & 0.003 \\
\hline & $95 \%$ HPD & $\begin{array}{l}(27.9, \\
29.9)\end{array}$ & $\begin{array}{l}(381 \mathrm{E} 3, \\
385 \mathrm{E} 3)\end{array}$ & $\begin{array}{l}(348.9, \\
352.6)\end{array}$ & $\begin{array}{l}(30.3, \\
37.3)\end{array}$ & & $\begin{array}{l}(0.031, \\
0.041)\end{array}$ \\
\hline \multirow{3}{*}{3} & Mean & 27.1 & $372 \mathrm{E} 3$ & 341.1 & 37.1 & 0.0004 & \\
\hline & STD & 0.5 & 848 & 0.8 & 9.2 & 0.0009 & \\
\hline & $95 \% \mathrm{HPD}$ & $\begin{array}{l}(26.1, \\
28.0)\end{array}$ & $\begin{array}{l}(371 \mathrm{E} 3, \\
374 \mathrm{E} 3)\end{array}$ & $\begin{array}{l}(339.6, \\
342.6)\end{array}$ & $\begin{array}{c}(19 ., \\
55.13)\end{array}$ & $\begin{array}{c}(-0.0014 \\
0.0023)\end{array}$ & \\
\hline
\end{tabular}

Figures $4.5,4.6$, and 4.7 show the samples of the structure parameters and the controller parameters. Results shown indicate that there is no correlation between the samples of the structural parameters and those of the controllers. This is an important finding because it indicates that the model of the human could be independent of the structural system.

The posterior predictive check of the models are shown in Figures 4.8, 4.9, and 4.10. As observed in the figures, the transfer function of the new experimental dataset falls 

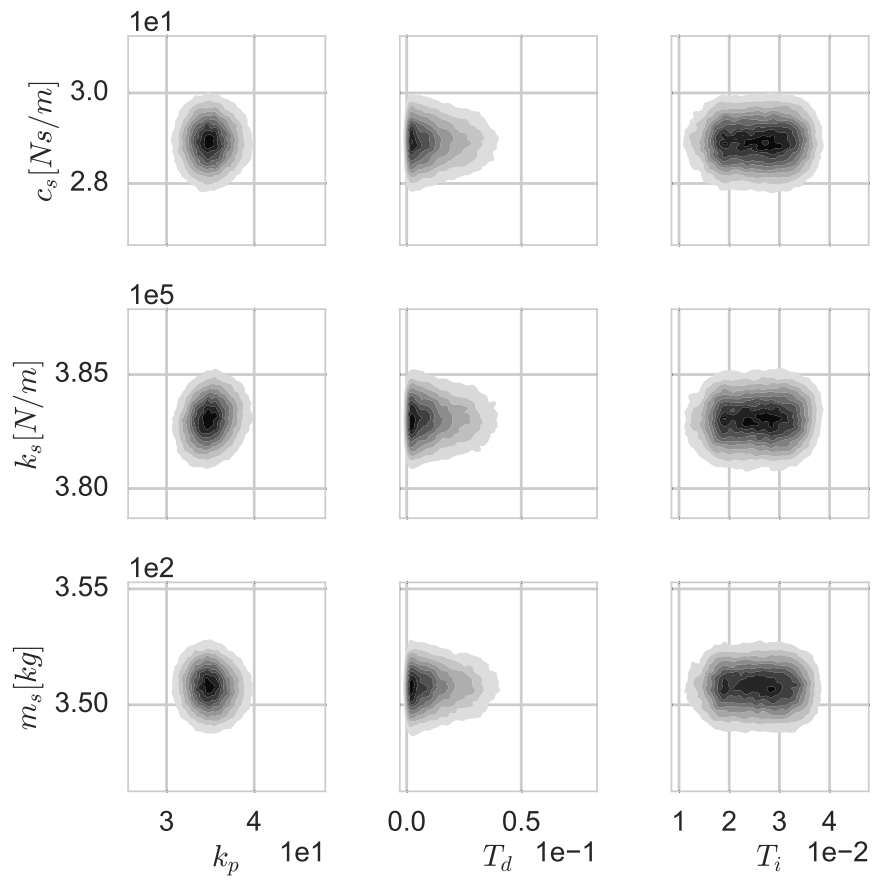

Figure 4.5: Samples for PID controller and structure's parameters
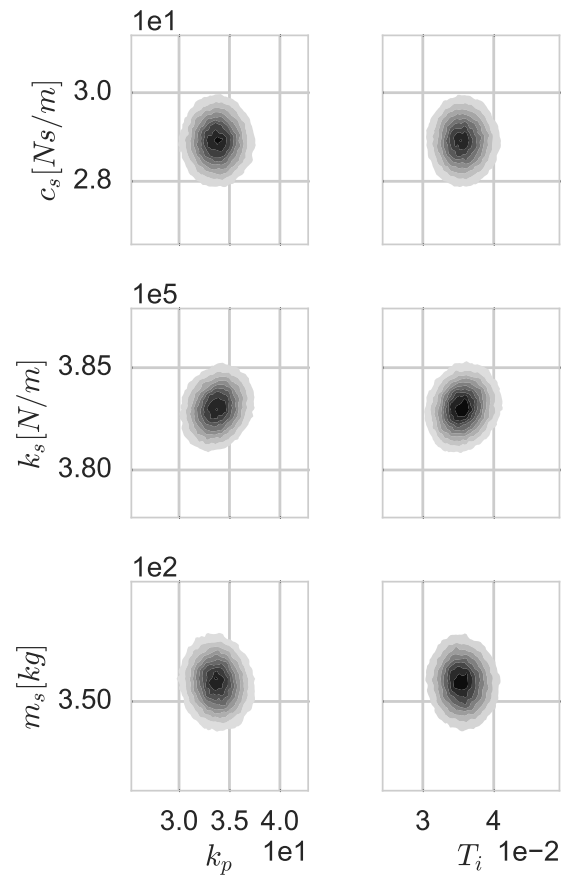

Figure 4.6: Samples for PI controller and structure's parameters 

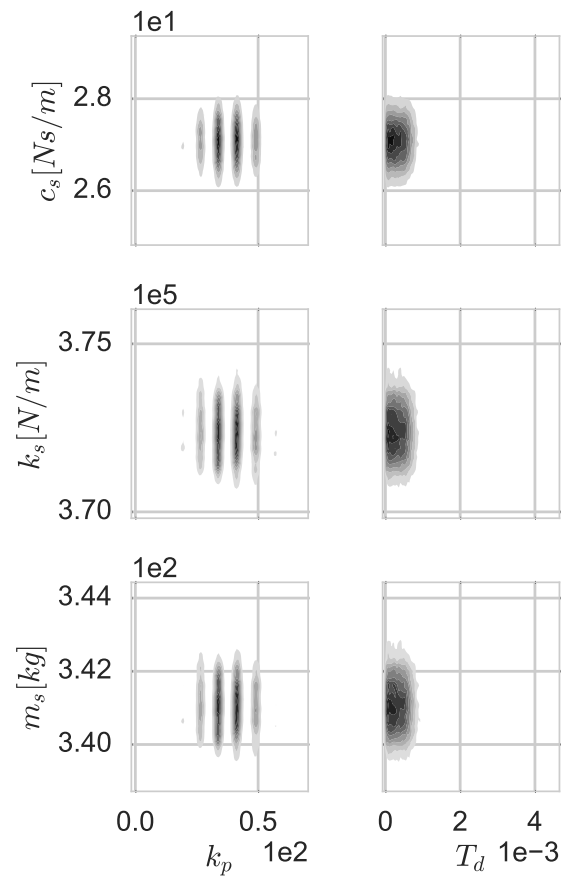

Figure 4.7: Samples for PD controller and structure's parameters

within the $95 \%$ HPD interval of the models. However, the confidence interval of model 3 is significantly wider than the confidence interval of models 1 and 2 . The confidence interval of PID and PI models are similar; therefore, the additional term $t_{d}$ in the PID controller is not affecting the confidence interval of the transfer function of the system. 


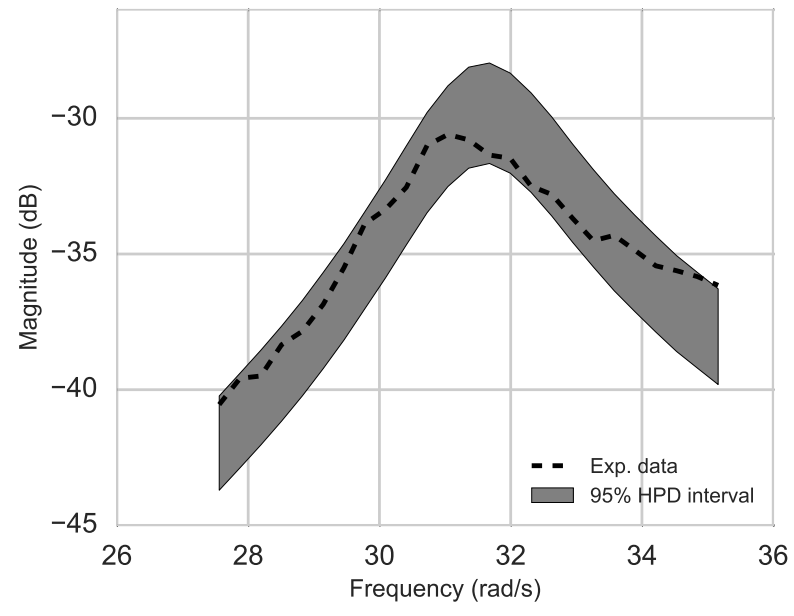

Figure 4.8: Posterior predictive check of model 1

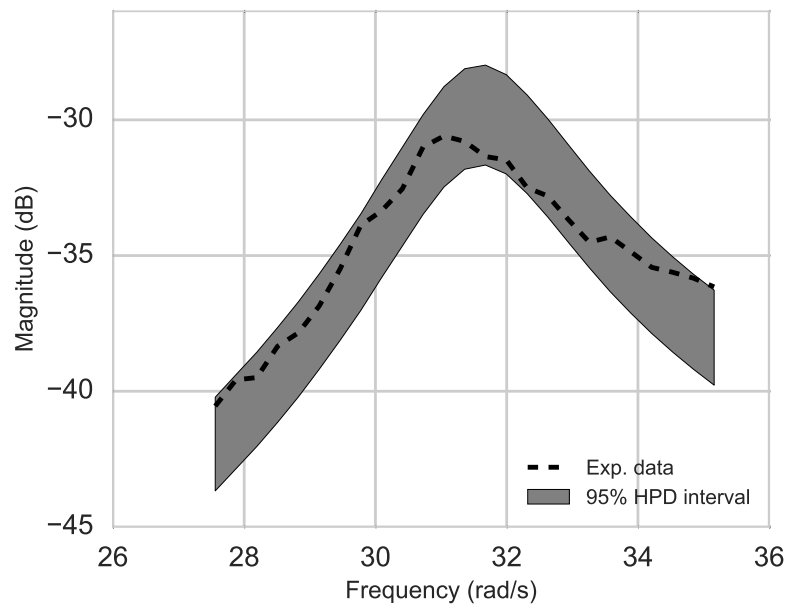

Figure 4.9: Posterior predictive check of the model 2 


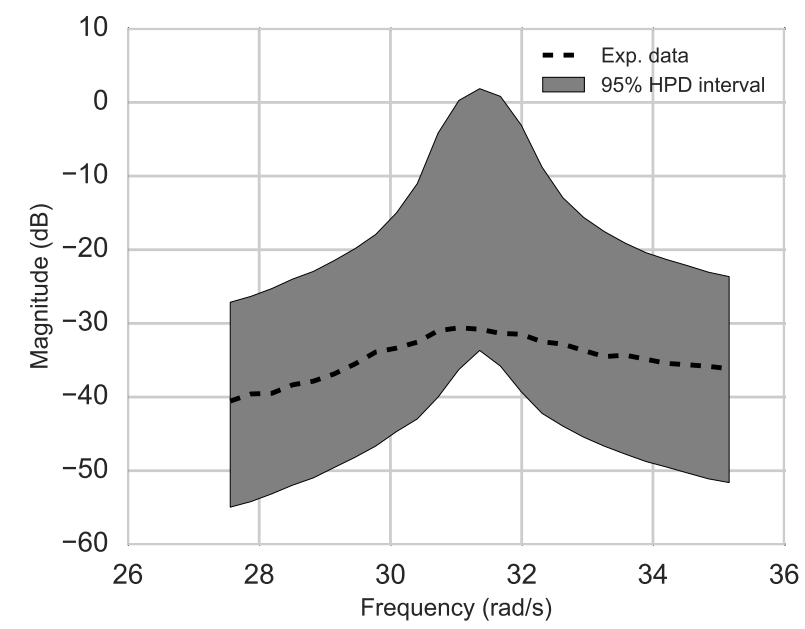

Figure 4.10: Posterior predictive check of the model 3

\subsubsection{Model 4: MCK ANd Single Degree of Freedom System}

This model represents the structure with the mass, damping, and stiffness parameters and proposes the use of a single degree of freedom model for modeling the human. The convergence of this model was reached using 150,000 samples, after burning 150,000. The Geweke z-score plots describing the convergence of the MCMC chains can be found in pages 89-93 of Appendix B.

The prior probability distributions of the stiffness, $k_{h}$, and the damping constant, $c_{h}$, are based on the parameters found in the literature, including those reported in the left column of the Table 1.2. The prior distribution for the mass of the human, $m_{s}$, is a normal distribution with mean $72 \mathrm{~kg}$ and standard deviation of $1 \mathrm{~kg}$. Several papers have stated the natural frequency of the human for a standing position; therefore, a

prior distribution was used for the natural frequency of the human, $\omega_{h}=\sqrt{k_{h} / m_{s}}$. Table 4.6 shows the prior used for updating the model.

Figure 4.11 shows the samples for the parameters of the human model. No dependence is found although the natural frequency of the human, $\omega_{h}$ is slightly correlating the parameters $c_{h}$ and $k_{h}$. The mean, standard deviation and the 95\% HPD inter- 
Table 4.6: Prior definition for parameters of the SDOF model of the human

\begin{tabular}{ccc}
\hline Parameter & Distribution & Values \\
\hline$k_{h}$ & Uniform & min $=100 \mathrm{~N} / \mathrm{m}, \max =100000 \mathrm{~N} / \mathrm{m}$ \\
$m_{h}$ & Normal & $\mu=72 \mathrm{~kg}, \sigma=1 \mathrm{~kg}$ \\
$c_{h}$ & Uniform & $\min =100 \mathrm{Ns} / \mathrm{m}, \max =100000 \mathrm{Ns} / \mathrm{m}$ \\
$\omega_{h}$ & Gamma & $\alpha=8.143, \beta=1.429$ \\
\hline
\end{tabular}

val of the samples are shown in Table 4.7. Figure 4.12 shows the parameters of the structure and the parameters of the human model. No dependence is observed.

Table 4.7: Moments of random variables describing the parameters of the model 4

\begin{tabular}{ccccccc}
\hline Parameter & $c$ & $k$ & $m$ & $c_{h}$ & $k_{h}$ & $m_{h}$ \\
& {$\left[\frac{N-s}{m}\right]$} & {$[N / m]$} & {$[k g]$} & {$\left[\frac{N-s}{m}\right]$} & {$[N / m]$} & {$[k g]$} \\
\hline Mean & 28.9 & $382.7 \mathrm{E} 3$ & 350.6 & 1963.8 & 1864.9 & 70.4 \\
STD & 0.5 & 1000.0 & 0.92 & 80.6 & 812.4 & 0.9 \\
95\% HPD & $(27.9$, & $(380.8 \mathrm{E} 3$, & $(348.8$, & $(1805.7$, & $(453.3$, & $(68.5$, \\
& $29.8)$ & $384.7 \mathrm{E} 3)$ & $352.4)$ & $2120.1)$ & $3468.9)$ & $72.2)$
\end{tabular}

The posterior predictive check of the model is shown in Figure 4.13. As observed in the figure, the transfer function of the new experimental dataset falls within the 95\% HPD interval of the predicted model. 

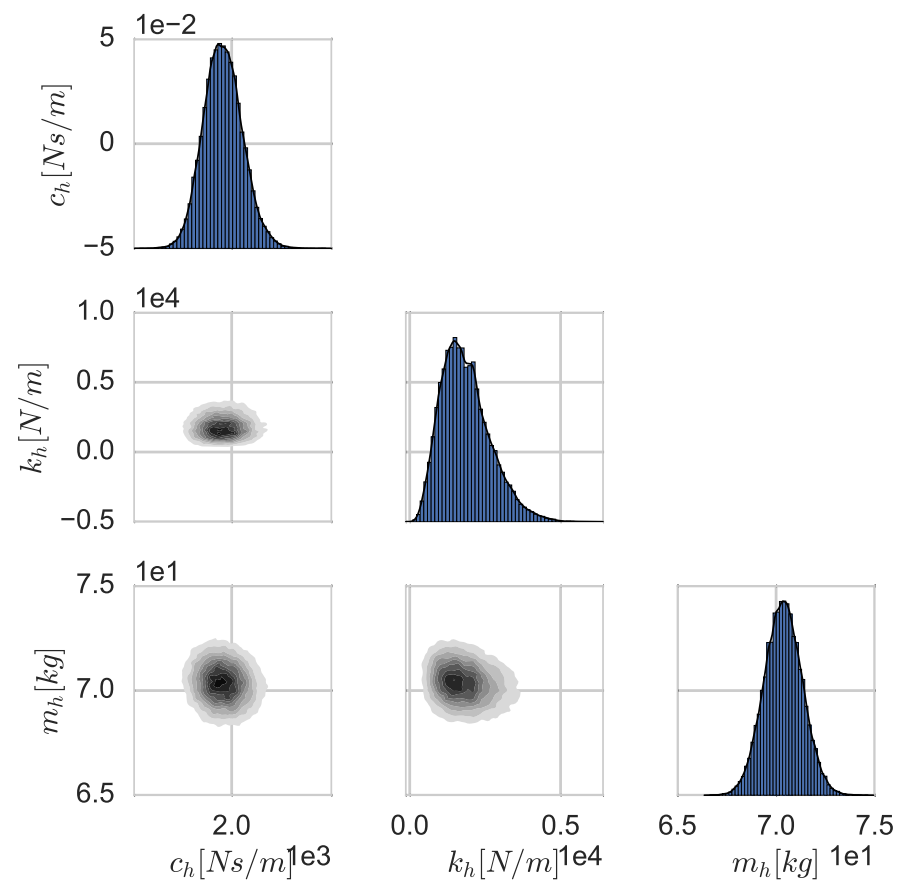

Figure 4.11: Marginal histogram (diagonal) and samples for the SDOF model of the human
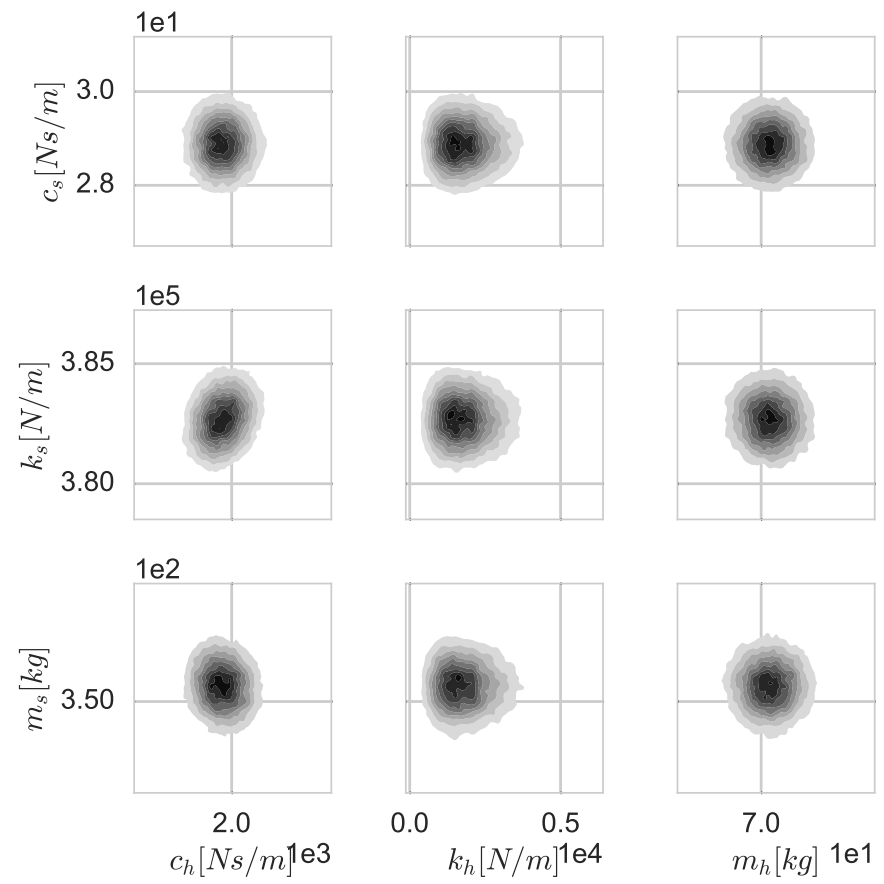

Figure 4.12: Samples for SDOF model of the human and structure's parameters 


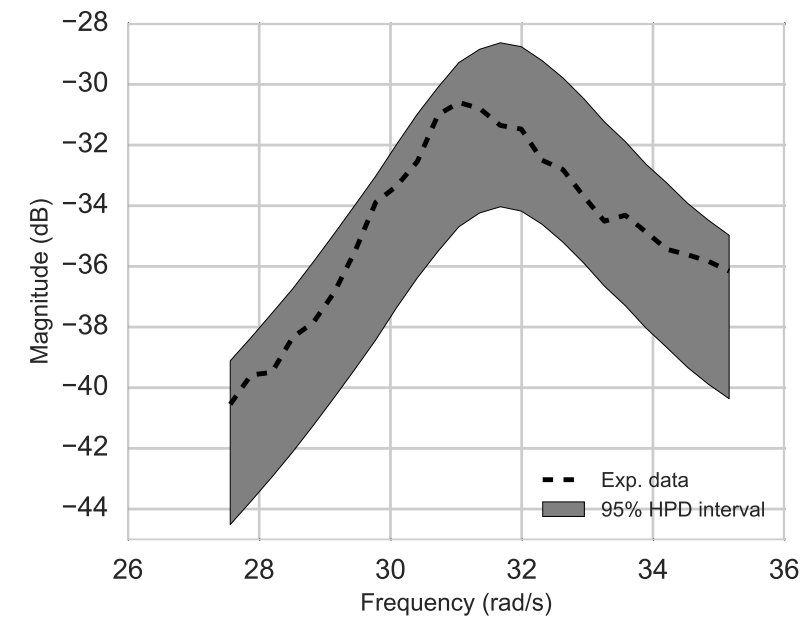

Figure 4.13: Posterior predictive check of the model 4

\subsubsection{Model 5: MCK AND Two DEgrees OF FREEDOM SyStem}

This model represents the structure with the mass, dampers and stiffness of a single degree of freedom system, and proposes the use of a two degree of freedom system for modeling the human. The convergence of this model was reached using 150,000 samples, after burning 150,000. The Geweke z-score plots describing the convergence of the MCMC chains can be found in pages 93-98 of Appendix B.

The prior probability density functions of the parameters is based on the values found in the literature and shown in the right column of the Table 1.2. Additional priors were defined in order to include the total mass of the human, and the first natural frequency of the human body. The total mass of the human, $m_{t}$, is expressed as equation 4.1. The prior of $m_{t}$ follows a normal distribution with mean $\mu=72 \mathrm{~kg}$ and $\sigma=1.0$.

$$
m_{t}=m_{h 1}+m_{h 2}
$$

The first natural frequency of the human body follows a distribution as presented in the last section. A uniform distribution is used to constrain the damping ratio 
of the two vibration modes of the human body between $10 \%$ and $60 \%$. Table 4.8 summarizes the prior used for updating the model.

Table 4.8: Prior definition for parameters of the 2DOF model of the human

\begin{tabular}{ccc}
\hline Parameter & Distribution & Values \\
\hline$k_{h 1}$ & Normal & $\mu=175.8 \mathrm{kN} / \mathrm{m}, \sigma=105.4 \mathrm{kN} / \mathrm{m}$ \\
$m_{h 1}$ & Normal & $\mu=55.4 \mathrm{~kg}, \sigma=9.8 \mathrm{~kg}$ \\
$c_{h 1}$ & Normal & $\mu=2330.0 \mathrm{Ns} / \mathrm{m}, \sigma=620.0 \mathrm{Ns} / \mathrm{m}$ \\
$k_{h 2}$ & Normal & $\mu=45.1 \mathrm{kN} / \mathrm{m}, \sigma=26.6 \mathrm{kN} / \mathrm{m}$ \\
$m_{h 2}$ & Normal & $\mu=16.1 \mathrm{~kg}, \sigma=9.81 \mathrm{~kg}$ \\
$c_{h 2}$ & Normal & $\mu=622.0 \mathrm{Ns} / \mathrm{m}, \sigma=380.0 \mathrm{Ns} / \mathrm{m}$ \\
$\omega_{h 1}$ & Gamma & $\alpha=8.143, \beta=1.429$ \\
$m_{t}$ & Normal & $\mu=72.0 \mathrm{~kg}, \sigma=1.0 \mathrm{~kg}$ \\
\hline
\end{tabular}

Tables 4.9 and 4.10 show the mean, standard deviation and the 95\% HPD interval of the samples.

Table 4.9: Moments of random variables describing the parameters of the model 5

\begin{tabular}{ccccccc}
\hline Parameter & $c$ & $k$ & $m$ & $c_{h 1}$ & $k_{h 1}$ & $m_{h 1}$ \\
& {$\left[\frac{N-s}{m}\right]$} & {$[N / m]$} & {$[k g]$} & {$\left[\frac{N-s}{m}\right]$} & {$[N / m]$} & {$[k g]$} \\
\hline Mean & 28.9 & $383.1 \mathrm{E} 3$ & 350.7 & 799.4 & 11728.4 & 41.6 \\
STD & 0.5 & 1002.4 & 0.9 & 34.4 & 1025.3 & 1.7 \\
95\% HPD & $(27.9$, & $(381.3 \mathrm{E} 3$, & $(348.9$, & $(730.9$, & $(9758.0$, & $(38.4$ \\
& $29.9)$ & $385.2 \mathrm{E} 3)$ & $352.5)$ & $867.7)$ & $13804.9)$ & $44.9)$
\end{tabular}

Table 4.10: Moments of random variables describing the parameters of the model 5 (Cont.)

\begin{tabular}{cccc}
\hline Parameter & $\begin{array}{c}c_{h 2} \\
{\left[\frac{N-s}{m}\right]}\end{array}$ & $\begin{array}{c}k_{h 2} \\
{[N / m]}\end{array}$ & $\begin{array}{c}m_{h 2} \\
{[\mathrm{~kg}]}\end{array}$ \\
\hline Mean & 591. & 62242.4 & 30.4 \\
STD & 341. & 20781.3 & 1.8 \\
95\% HPD & $(17.2$, & $(21615.6$, & $(27$. \\
& $1183.2)$ & $106370.9)$ & $33.9)$
\end{tabular}

Figures $4.14,4.15$, and 4.16 show the samples for the parameters of the 2DOF human model. A strong dependence is found between $m_{h 1}$ and $m_{h 2}$ as expected.

Figure 4.17 and 4.18 show the parameters of the structure and the parameters of the human model. There are no correlations found. 

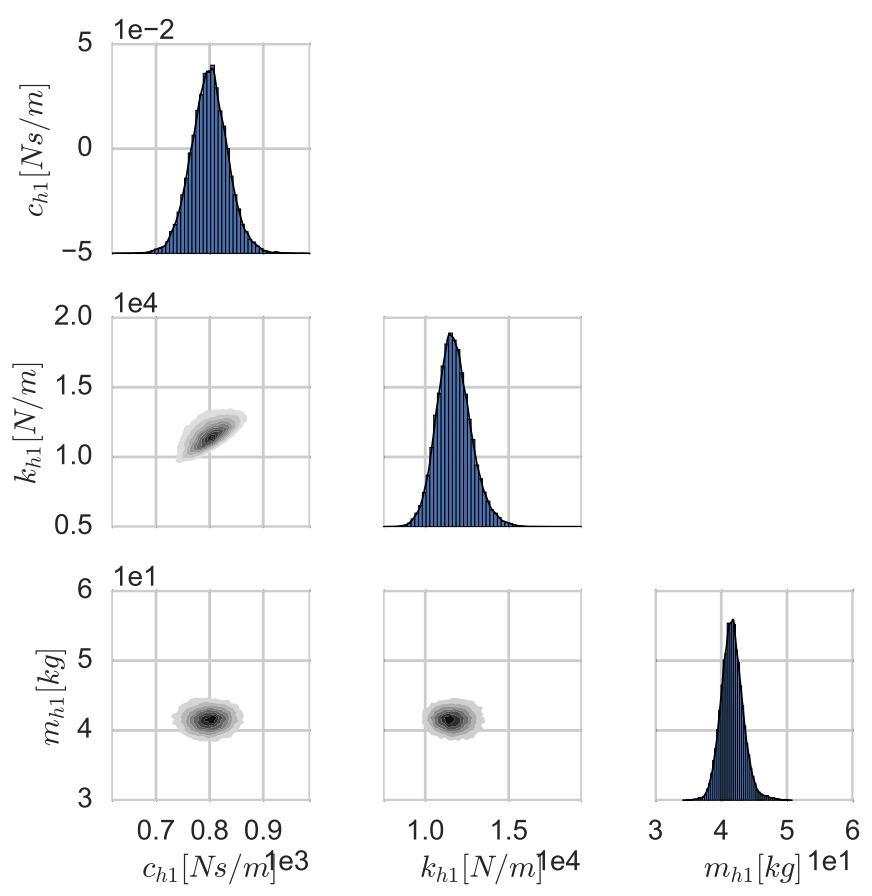

Figure 4.14: Marginal histogram (diagonal) and samples for the 2DOF model of the human (Figure 1 of 3 )

The posterior predictive check of the model is shown in Figure 4.19. The transfer function of the new experimental dataset falls within the $95 \%$ HPD interval of the model. 

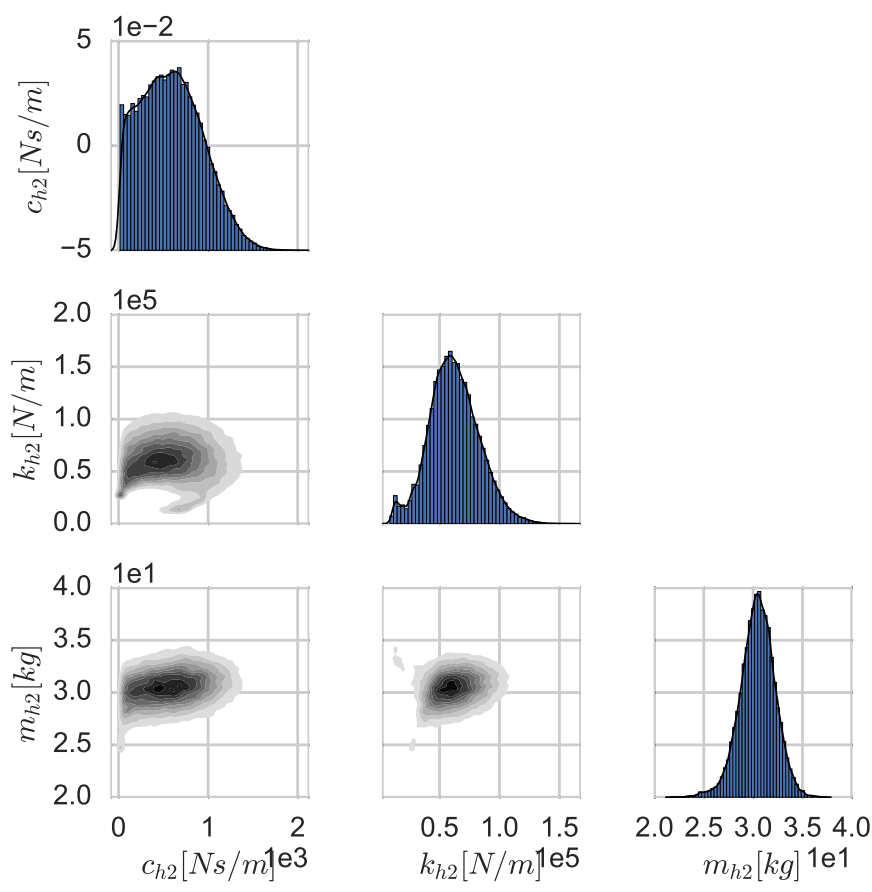

Figure 4.15: Marginal histogram (diagonal) and samples for the 2DOF model of the human (Figure 2 of 3 )
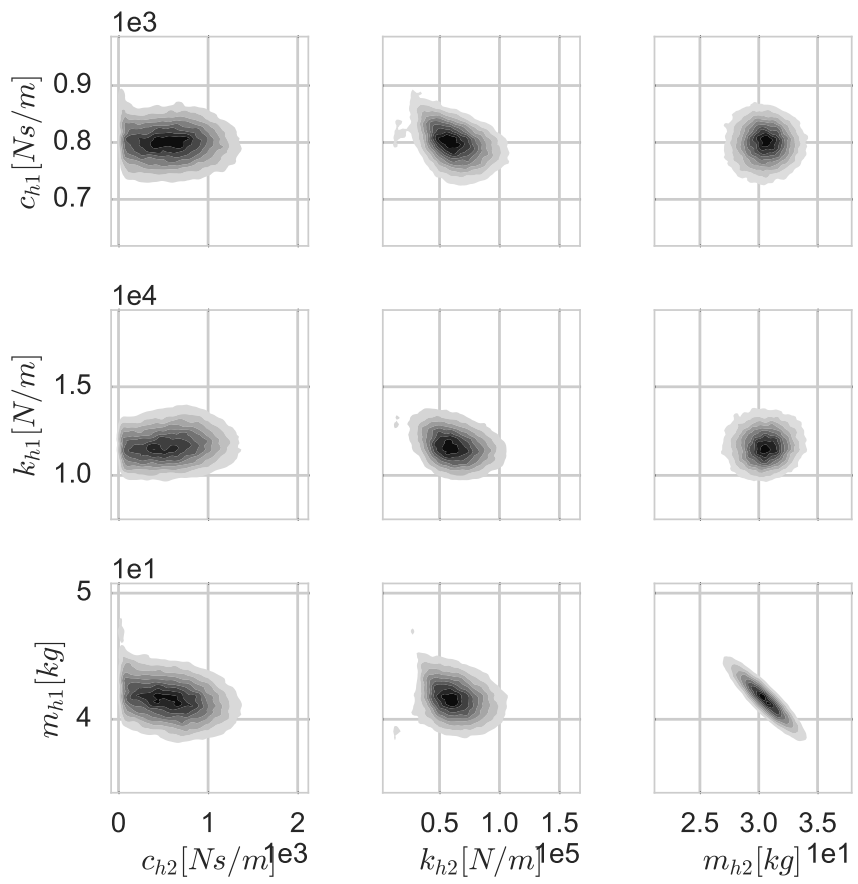

Figure 4.16: Samples for the 2DOF model of the human (Figure 3 of 3) 

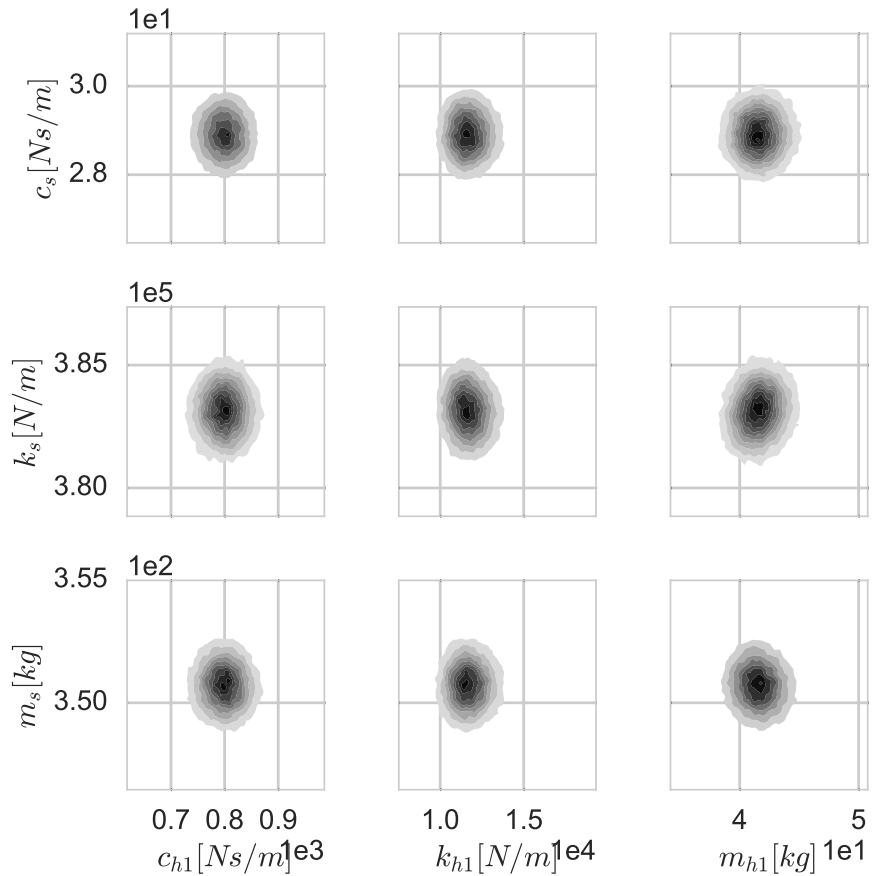

Figure 4.17: Samples for 2DOF model of the human and structure parameters (Figure 1 of 2 )
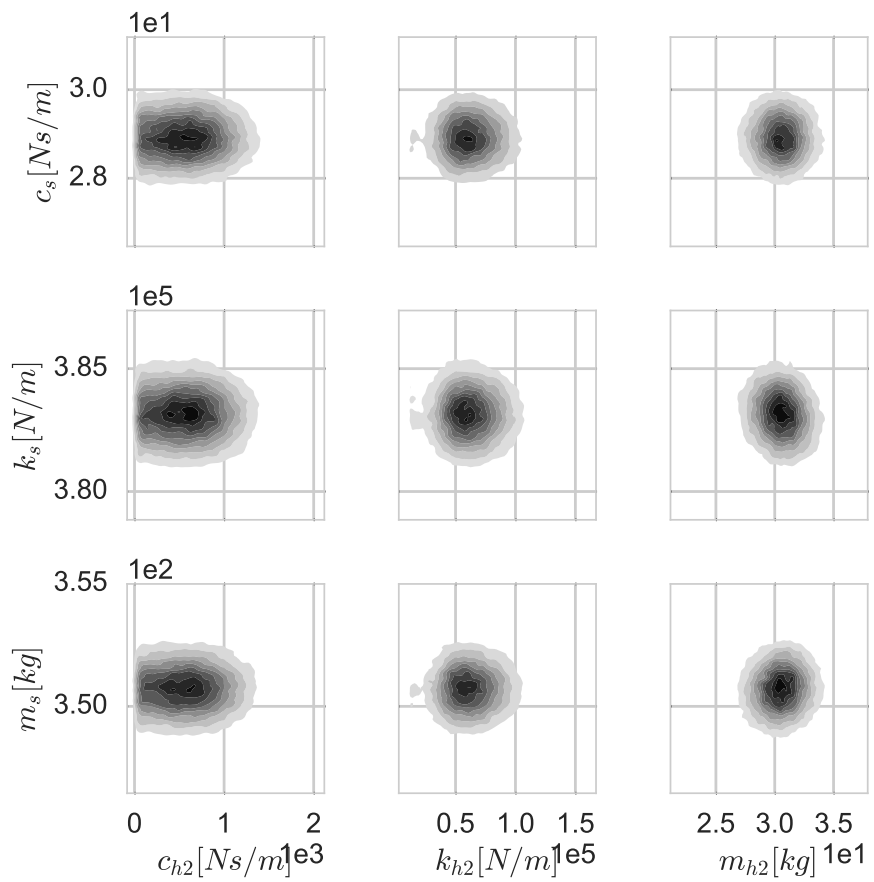

Figure 4.18: Samples for 2DOF model of the human and structure parameters (Figure 2 of 2 ) 


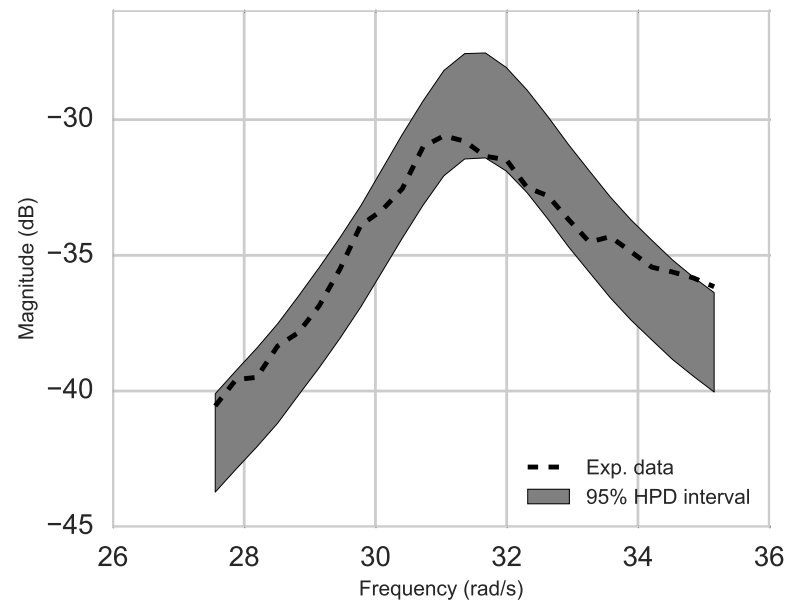

Figure 4.19: Posterior predictive check of the model 5 


\subsection{Model SELECTION}

Controller and traditional models are compared using the probabilistic approach described in Chapter 2. The comparison is based on the Bayes factor, which is a ratio between the evidence of the models. The mean of 20 independent iterations is used to estimate the integral for each model. Each iteration contains $n$ evaluations of the integrand. A convergence analysis is performed for each model in order to find the minimum number of evaluations to perform in each interaction. Figure 4.20 shows an example of the convergence analysis for model 5. The number of iterations used in this case is $10^{5}$ because results of the integral do not change, and the standard deviation is small, around $1 \%$ of the value obtained.

Table 4.11 shows the number of evaluations used, the mean, and the coefficient of variation $(\mathrm{CV})$ of the integration for each one of the five models used for modeling human-structure interaction.

Table 4.12 shows the Bayes factor based on the values reported in Table 4.11. The

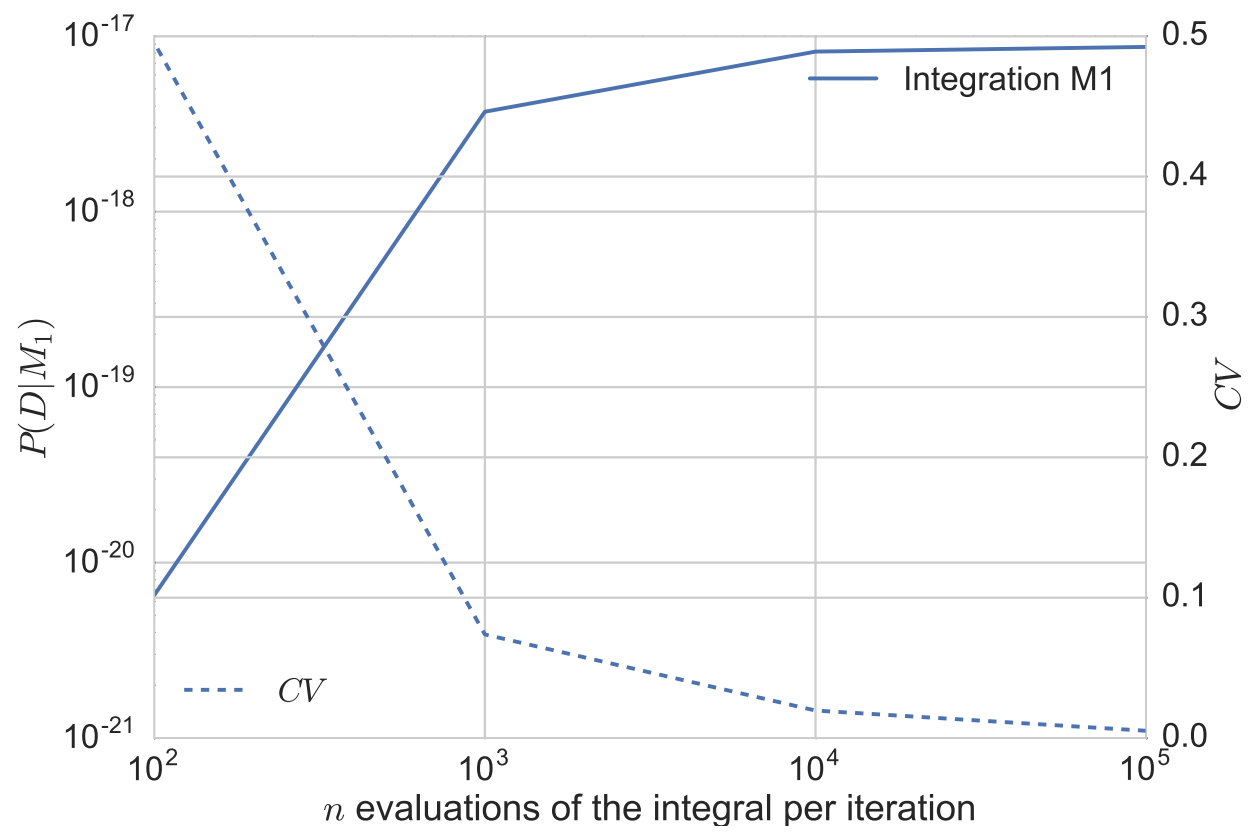

Figure 4.20: Converegence of the integration 
Table 4.11: Results of the integration of human-structure interaction models

\begin{tabular}{cccc}
\hline Model & $\begin{array}{c}\text { Number of } \\
\text { evaluations }\end{array}$ & Mean & $\begin{array}{c}\text { Coe. of Variation } \\
\%\end{array}$ \\
\hline 1 & $10^{5}$ & $8.68 \mathrm{E}-18$ & 0.4 \\
2 & $10^{5}$ & $1.39 \mathrm{E}-17$ & 0.1 \\
3 & $10^{5}$ & $3.76 \mathrm{E}-40$ & 0.1 \\
4 & $10^{5}$ & $4.54 \mathrm{E}-19$ & 0.1 \\
5 & $10^{5}$ & $6.74 \mathrm{E}-20$ & 0.5 \\
\hline
\end{tabular}

rows are the numerator of the equation and the columns are the denominators.

Table 4.12: Classificacion of human-structure interaction models for Bayes factor, $2 \operatorname{Ln}(K)$

\begin{tabular}{c|cccc}
\hline & \multicolumn{4}{|c}{ Model $(j)$} \\
Model $(i)$ & 1 (PID) & 4 (SDOF) & $5(\mathrm{TDOF})$ & $3(\mathrm{PD})$ \\
\hline 2 (PI) & 0.94 & 6.84 & 10.68 & 103.93 \\
1 (PID) & & 5.90 & 9.716 & 102.99 \\
4 (SDOF) & & & 3.82 & 97.09 \\
5 (TDOF) & & & & 93.27 \\
\hline
\end{tabular}

Table 4.13: Classificacion for human-structure interaction models based on Kass and Raftery scale [1]

\begin{tabular}{c|cccc}
\hline & \multicolumn{4}{|c}{ Model $(j)$} \\
Model $(i)$ & 1 (PID) & 4 (SDOF) & $5($ TDOF $)$ & $3(\mathrm{PD})$ \\
\hline 2 (PI) & negligible & Strong & Very strong & Very strong \\
1 (PID) & & Positive & Strong & Very strong \\
4 (SDOF) & & & Positive & Very strong \\
5 (TDOF) & & & & Very strong \\
\hline
\end{tabular}

The PI controller has the highest probability when compared with the other models considered in this work. Based on the Kass and Raftery scale [1] shown in 4.13, there is "not worth than a bare mention" evidence to prefer the PI over the PID. However, there is a strong and very strong evidence to prefer the PI over the SDOF and 2DOF respectively. Out of the MDS models, there is a positive evidence to prefer the SDOF over the 2DOF. The preference of the PI over the PID and the SDOF 
over the $2 \mathrm{DOF}$ is related to the number of parameters in each model as discussed in chapter 2.

Results show that the PI and PID models could more likely represent the humanstructure interaction phenomenon than the traditional MDS models. The fact that these are new models produce uncertainties in the definition of the prior of the parameters, which are reflected in the calculation of the model evidence. These uncertainties could be further reduced if more research is conducted.

Table 4.12 also shows that the PD controller has the lowest probability of all models. Its evidence has a ratio of around $20+$ order of magnitudes to the evidence of other models. One possible interpretation of these results is that the velocity of the structure plays an important role in the human-structure interaction problem. The PI and the PID models, which include velocity, have a much higher probability than the PD, which does not.

\subsection{Controller models For Groups of PeOple}

Tests with three different people were performed over the Configuration B of the structure. The goal of using different people is to find whether or not the controller models can be used for modeling human-structure interaction of groups. Table 4.14 summarizes the properties of the people involved in tests.

Table 4.14: Characteristics of the people involved in tests

\begin{tabular}{ccc}
\hline Person ID & $\begin{array}{c}\text { Height } \\
{[\mathrm{cm}]}\end{array}$ & $\begin{array}{c}\text { Mass } \\
{[\mathrm{kg}]}\end{array}$ \\
\hline P1 & 173 & 73 \\
P2 & 168 & 67 \\
P3 & 178 & 72 \\
\hline
\end{tabular}

Table 4.15 shows the moments of the $k_{p}$ and $t_{i}$ parameters after updated. The parameter $k_{p}$ has less variability than the parameter $t_{i}$. This means that $k_{p}$ is less susceptible to the changes of the human body than the $t_{i}$ parameter. Notice that 
the moments of these parameters are different to the those found for the $t_{i}$ model in the previous section (Model 2). This is explained because the structure used is the Configuration B which has two vibration modes, one vertical and the other with a strong horizontal component. Therefore, the human is also controlling the lateral vibration mode of the structure. Figure 4.21 shows the posterior predictive checks for people P1, P2, and P3.

Table 4.15: Moments of variables describing the PI controller for the people P1, P2 and P3

\begin{tabular}{cccc}
\hline \multirow{2}{*}{ Person ID } & Parameter & $K_{p}$ & $t_{i}$ \\
\hline & Mean & 121.1 & 1.230 \\
P1 & STD & 3.8 & 1.710 \\
& $95 \%$ HPD & $(113.6$, & $(0.328$, \\
& & $128.5)$ & $5.532)$ \\
\hline \multirow{3}{*}{ P2 } & Mean & 129.8 & 0.218 \\
& STD & 7.75 & 0.073 \\
& 95\% HPD & $(114.9$, & $(0.112$, \\
& $145.6)$ & $0.359)$ \\
\hline \multirow{3}{*}{ P3 } & Mean & 123.7 & 0.576 \\
& STD & 4.3 & 0.344 \\
& 95\% HPD & $(115.5$, & $(0.235$ \\
& & $132.7)$ & $1.063)$ \\
\hline
\end{tabular}

The samples of the PI controllers found for individuals are used to predict the transfer function when two people are standing on the structure. The combinations studied are P1P2, P1P2 and P1P3. The predicted transfer functions are then compared with the corresponding experimental data. The transfer function of the system is calculated using the equation 2.8. In this case, two humans $(n=2)$ are over the structure, therefore the equation 2.8 is as follows:

$$
T F(s)=\frac{G(s)}{1+G(s)\left[H_{1}(s)+H_{2}(s)\right]}
$$

The results are shown in Figure 4.22. The predicted transfer functions show some discrepancy with the experimental results although most of the experimental transfer 


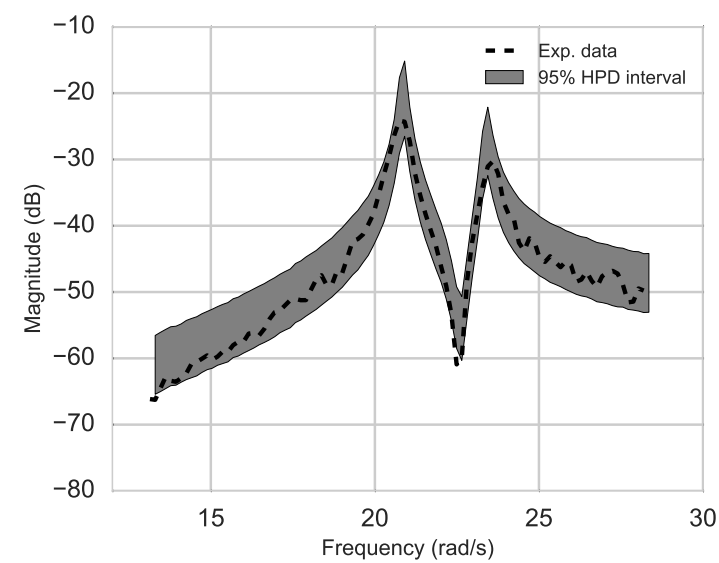

(a)

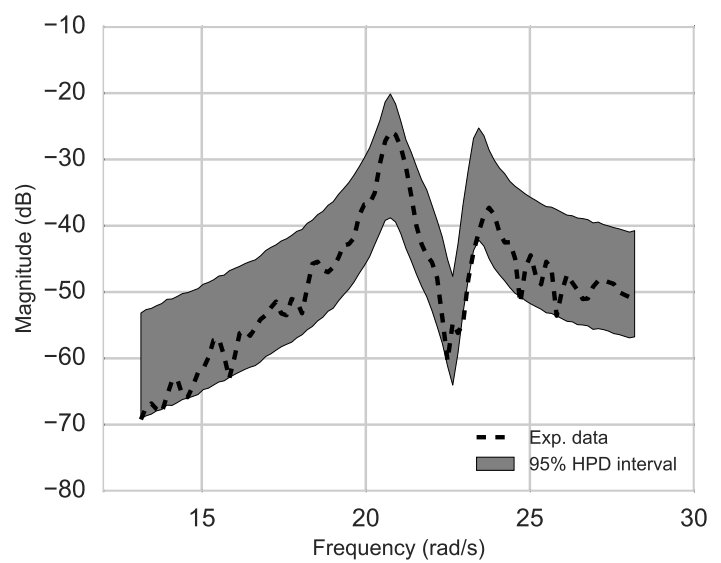

(b)

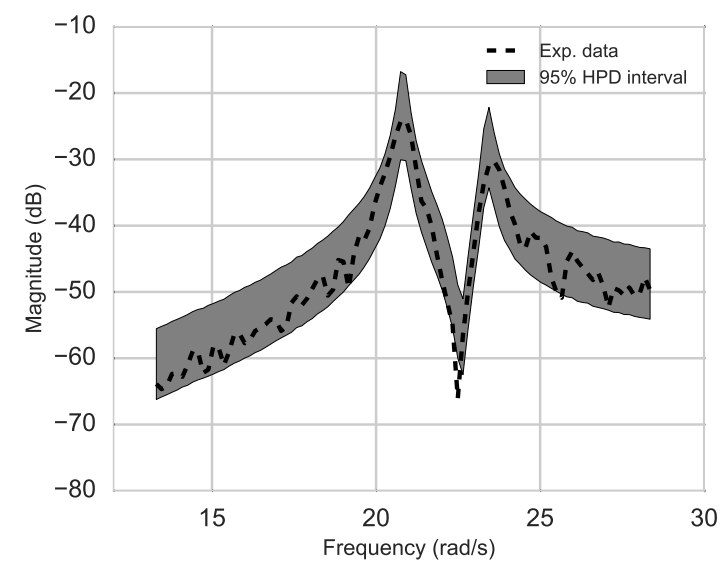

(c)

Figure 4.21: Posterior predictive checks of PI models for each single occupant

function fall in the $95 \%$ confidence intervals. The transfer function of two humans over the same structure is slightly different to the transfer function calculated with the sum of controllers representing each human. This means that the controller should not be actuating perfectly in parallel, and some feedback between the human is affecting the results. 


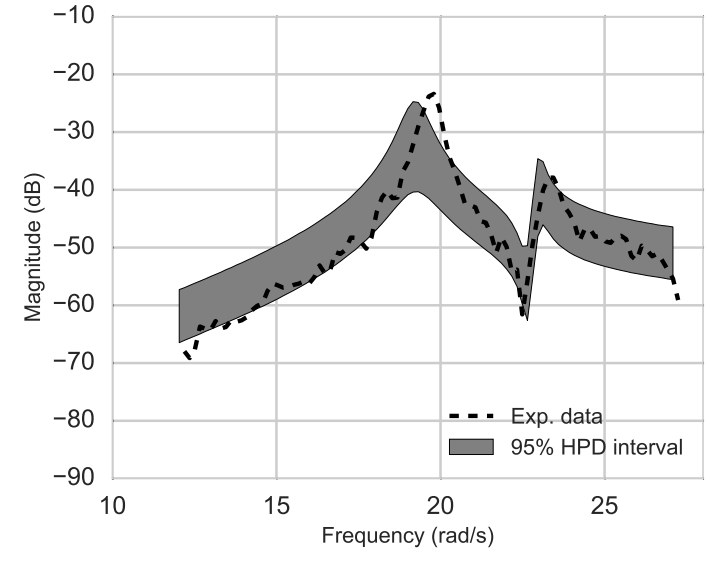

(a)

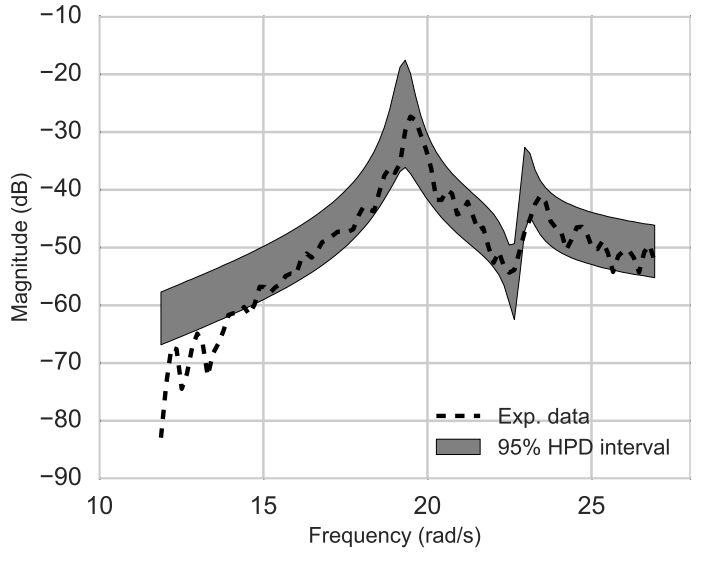

(b)

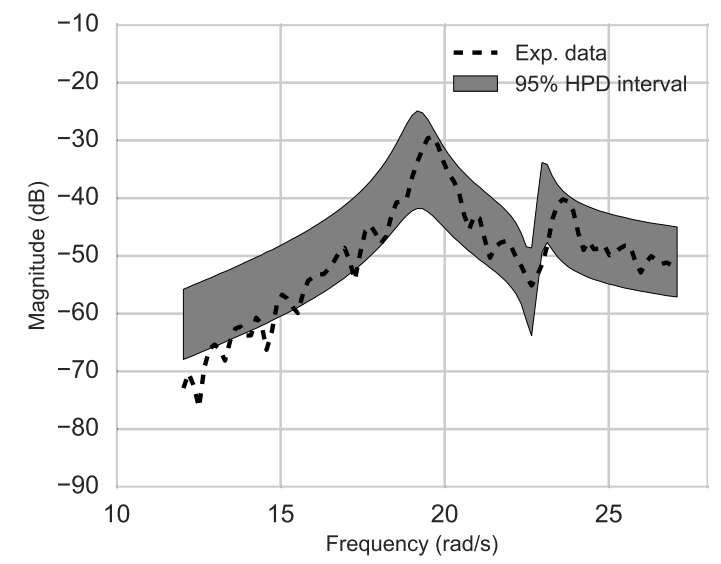

(c)

Figure 4.22: Predictive checks for groups of two humans 


\section{Chapter 5 \\ CONCLUSIONS AND FUTURE WORK}

\subsection{Conclusions}

The focus of this research is to introduce a new approach based on a closed-loop control system for modeling the human-structure interaction phenomenon. Within this approach, the human is modeled as a controller system and the structure as the system to control. Controller models seek to include a stronger feedback action between the structure and the human than the traditional mass-damper-spring (MDS) models. This approach may help explain the changes of the dynamic characteristics experienced in structures occupied by crowds. This work focuses on standing individuals but the framework used to update and compare the models can be applied to other cases.

This research shows that the idea of modeling a standing individual as a controller works. This is evidenced by comparing the models in a probabilistic way. There is strong evidence than a PI model is preferred over traditional MDS models.

Three different controllers were updated using a Bayesian probabilistic approach. The Proportional, Derivative, and Integrative controller, PID, and the proportional and integrative controller, PI, were able to successfully model the phenomenon. However, the third controller evaluated, the PD, did not show good fit with the experimental data.

The PI controller has the strongest evidence among the models evaluated. Results indicate that the integrative term, $t_{i}$, is significant to the controller. The $t_{i}$ param- 
eter changes the controller output based on the velocity of the structure, which is consistent with findings by other authors [36].

Groups of people were modeled using the controller models identified for each person. The model assumed that the controllers work independently of each other. Under this assumption, the modeling of groups was explored and its performance was acceptable. However, peaks of the model's transfer function are at a slight different frequency from the peaks of the experimental transfer function. This could be due to of human-human interactions not modeled.

The Bayesian probabilistic model updating technique enabled the identification of the parameter's probability density functions, as well as correlations between the parameters of human and structure models. These correlations are difficult to predict from the deterministic models described in the literature review presented in Chapter 1. Controller models, in general, were found to be independent of the structure, which means that the parameters of the controller could be developed in a lab and implemented in other structural models, as long as the stability conditions are satisfied.

It is important to consider all the stability conditions of the system because they can strongly affect the performance of a controller. For example, the PD controller makes the structure unstable when using parameters values near to high probability regions. Stability conditions are big constraints of the controller models, and their formulation lead to additional equations that could reduce the simplicity of the models.

\subsection{Future Work}

The main goal of this research was to explore the use of closed-loop control theory to model the human-structure interaction problem. It is promising that the PI controller has substantial strength in the evidence with respect to the traditional MDS 
models. Additional work in this field will reduce the model uncertainty by reducing the probability density function of the priors, which will make the evidence of these models even higher.

The selected controllers PID, PD, and PI are just a family of controllers of hundreds found in the literature [38]. The exploration of better controllers could reduce the uncertainty in modeling the phenomenon. For example, adaptive controllers could introduce the variability of the human body given some characteristics such as the mass, height, age, or body mass index.

Standing humans were used to validate the methodology applied to this research. However, problems in the human-structure interaction also involve humans in movement, such as walking and running. The implementation of controller models for this kind of movements is the natural next step in this research. For modeling a pedestrian, the controller and biomechanical model of the human should be combined. The human-structure interaction in bridges could be explored, for example, using a combination of the inverted pendulum model of the human body with the controller actions to maintain stability. 


\section{BIBLIOGRAPHY}

[1] Robert E Kass and Adrian E Raftery. Bayes factors. Journal of the american statistical association, 90(430):773-795, 1995.

[2] A. Fitzpatrick, P. Dallard, A. Low, S. Le Bourva, R. Ridsdill, and M. Willford. Linking London: The Millennium Bridge. Royal Academy of Engineering, London, UK, 2001.

[3] P. Dallard, A. Fitzpatrick, A. Flint, S. Le Bourva, R. Ridsdill, and M. Willford. The london millennium footbridge. The Structural Engineer, 79:17-33, 2009.

[4] B. S. Morris. An Investigation of Human Induced Vibrations on Clemson Memorial Stadium. Master of Science Thesis. Clemson University, 2009.

[5] S. Erlingsson A. Bodare. Rock music induced damage and vibration at nya ullevi stadium. Procedings: Third International Conference on Case Histories in Geotechnical Engineering, St. Louis, Missouri, pages 671-675, 1993.

[6] JHG Macdonald. Pedestrian-induced vibrations of the clifton suspension bridge, uk. Proceedings of the ICE-Bridge Engineering, 161(2):69-77, 2008.

[7] A. Ortiz, D. Gomez, and P. Thomson. Effect of human-structure interaction on the dynamic properties of a grandstand. Ingenieria y Competitividad, 14:63-73, 2012.

[8] S. Falati. The contribution of non-structural components to the overall dynamic behaviour of concrete floor slabs. University of Oxford, Oxford, UK, 1999.

[9] E. Agu and M. Kaspersky. Influence of the random dynamic parameters of the human body on the dynamic characteristics of the coupled system of structurecrowd. Journal of Sound and Vibration, 330:431-444, 2011.

[10] K. Salyards and Y. Hua. Assessment of dynamic properties of a crowd model human-structure interaction modeling. 89:103-110. 
[11] Proceedings of the Fourth International Conference on Structural Dynamics, editor. The influence of a group of humans on modal properties of a structure, volume 2, Munich, 2002. Pitman.

[12] N. W. M. Bishop, M. Willford, and R. Pumphrey. Human induced loading of flexible staircases. 18:261-276.

[13] R. Sachse, A. Pavic, and P. Reynolds. Human-structure dynamic interaction in civil engineering dynamics: A literature review. The Shock and Vibration Digest, 35:3-18, 2003.

[14] S. Zivanovic, A. Pavic, and P. Reynolds. Vibration serviceability of footbridges under human-induced excitation: a literature review. Journal of Sound and Vibration, 279:1-74, 2005.

[15] American Society of Civil Engineers ASCE. Minimum Design Loads for Buildings and Other Structures, ASCE/SEI 7-10. American Society of Civil Engineers ASCE, 2013.

[16] International Building Code Inc. International Building Code. International Building Code Inc., 2003.

[17] British Standards Institution (BSI). Code of practice for dead and imposed loads. American Society of Civil Engineers ASCE, 2011.

[18] Proceedings of the 31st International Modal Analysis Conference, editor. Experimental Results from a Laboratory Test Program to Examine Human-Structure Interaction. Springer, 2013.

[19] J.M.W. Brownjohn. Energy dissipation in one-way slabs with human participation. In Proceedings of the Asia-Pacific Vibration Conference $\hat{a} \breve{A} \ddot{Y} 99$, Singapore, December.

[20] ISO. Vibration and shock - Mechanical driving point impedance of the human body. International Organization for Standardization (ISO), 1981.

[21] L. Sim, A. Blackeborough, and M. Williams. Modelling of joint crowd structure system using equivalent reduced-dof system. Shock and Vibration, 14:261-270, 2007. 
[22] Y. Matsumoto and M.J. Griffin. Mathematical models for the apparent masses of standings subjects exposed to vertical whole-body vibration. Journal of Sound and Vibration, 260:431-451, 2003.

[23] Andrew L Smith, Stephen J Hicks, and Paul J Devine. Design of floors for vibration: A new approach. Steel Construction Institute Ascot, Berkshire, UK, 2007.

[24] Thomas M Murray. Floor vibrations: The human tolerance side of the equation. In SPIE proceedings series, pages 1-6. Society of Photo-Optical Instrumentation Engineers, 1999.

[25] Institution of Structural Engineers. Dynamic performance requirements for permanent grandstands subject to crowd action: Interim guidance on assessment and design. Institution of Structural Engineers, ISE, 2001.

[26] L. Wei and M.J. Griffin. Mathematical models for the apparent mass of the seated human body exposed to vertical vibration. Journal of Sound and Vibration, 212:855-874, 1998.

[27] A. Farah. Human response: A criterion for the assessment of structural serviceability.

[28] AJ Thurston. Giovanni borelli and the study of human movement: An historical review. Australian and New Zealand journal of surgery, 69(4):276-288, 1999.

[29] Frances A Hellebrandt, Rubye H Tepper, Genevieve L Braun, and Margaret C Elliott. The location of the cardinal anatomical orientation planes passing through the center of weight in young adult women. American Journal of PhysiologyLegacy Content, 121(2):465-470, 1938.

[30] G Elliot Smith. The human brain. Journal of the Royal Society of Arts, 78(4037):570-576, 1930.

[31] David A Winter. Human balance and posture control during standing and walking. Gait \& posture, 3(4):193-214, 1995.

[32] Itshak Melzer, N Benjuya, and J Kaplanski. Postural stability in the elderly: a comparison between fallers and non-fallers. Age and ageing, 33(6):602-607, 2004. 
[33] Yifa Jiang Hidenori Kimura. Balance-keeping control of upright standing in biped human beings and its application for stability assessment. INTECH Open Access Publisher, 2007.

[34] Taesoo Kwon and Jessica Hodgins. Control systems for human running using an inverted pendulum model and a reference motion capture sequence. In Proceedings of the 2010 ACM SIGGRAPH/Eurographics Symposium on Computer Animation, pages 129-138. Eurographics Association, 2010.

[35] Ian D Loram and Martin Lakie. Human balancing of an inverted pendulum: position control by small, ballistic-like, throw and catch movements. The Journal of physiology, 540(3):1111-1124, 2002.

[36] Kimura Hidenori and Yifa Jiang. A pid model of human balance keeping. Control Systems, IEEE, 26(6):18-23, 2006.

[37] Mateusz Bocian, John HG Macdonald, Jeremy F Burn, and David Redmill. Experimental identification of the behaviour of and lateral forces from freelywalking pedestrians on laterally oscillating structures in a virtual reality environment. Engineering Structures, 105:62-76, 2015.

[38] K. Ogata. Modern Control Engineering. Prentice-Hall, 2002.

[39] DJ Ewins. Modal testing: theory, practice and application (mechanical engineering research studies: engineering dynamics series). 2003.

[40] Graham Clifford Goodwin, Stefan F Graebe, and Mario E Salgado. Control system design, volume 240. Prentice Hall New Jersey, 2001.

[41] Albert R Ortiz and Juan M Caicedo. Comparing closed loop control models and mass-spring-damper models for human structure interaction problems. In Dynamics of Civil Structures, Volume 2, pages 67-74. Springer, 2015.

[42] John W Smith and Terry Montgomery. Biomechanically induced and controller coupled oscillations experienced on the f-16xl aircraft during rolling maneuvers. 1996.

[43] Hao Ying. Theory and application of a novel fuzzy pid controller using a simplified takagi-sugeno rule scheme. Information Sciences, 123(3):281-293, 2000. 
[44] Samuel Privara, Jan Širokỳ, Lukáš Ferkl, and Jiří Cigler. Model predictive control of a building heating system: The first experience. Energy and Buildings, 43(2):564-572, 2011.

[45] Shirley J Dyke, Juan Martin Caicedo, Gursoy Turan, LA Bergman, and Steven Hague. Phase i benchmark control problem for seismic response of cable-stayed bridges. Journal of Structural Engineering, 129(7):857-872, 2003.

[46] Pan Zhao, Jiajia Chen, Yan Song, Xiang Tao, Tiejuan Xu, and Tao Mei. Design of a control system for an autonomous vehicle based on adaptive-pid. International Journal of Advanced Robotic Systems, 9, 2012.

[47] Frederick Chee, Tyrone L Fernando, Andrey V Savkin, and Vernon Van Heeden. Expert pid control system for blood glucose control in critically ill patients. Information Technology in Biomedicine, IEEE Transactions on, 7(4):419-425, 2003.

[48] B Kada and Y Ghazzawi. Robust pid controller design for an uav flight control system. In Proceedings of the World Congress on Engineering and Computer Science, volume 2, pages 1-6, 2011.

[49] C Schwartz, A Berger, O Brüls, JL Croisier, B Forthomme, and V Denoël. Experimental study of the human ability to deliberately excite a flexible floor. Research and Applications in Structural Engineering, Mechanics and Computation, page 55, 2013.

[50] David E Newland. Vibration of the london millennium footbridge: Part 1-cause. University of Cambridge, 2003.

[51] J. Beck and L. S. Katafygiotis. Updating models and their uncertainties i: Bayesian statistical framework. Journal of Engineering Mechanics, 124:455-461, 2009.

[52] S. H. Cheung and J. Beck. Bayesian model updating using hybrid monte carlo simulation with application to structural dynamic models with uncertain parameters. Journal of Engineering Mechanics, 135:243-225, 2009.

[53] Christian Robert and George Casella. Monte Carlo statistical methods. Springer Science \& Business Media, 2013.

[54] W Keith Hastings. Monte carlo sampling methods using markov chains and their applications. Biometrika, 57(1):97-109, 1970. 
[55] Nicholas Metropolis and Stanislaw Ulam. The monte carlo method. Journal of the American statistical association, 44(247):335-341, 1949.

[56] John Geweke et al. Evaluating the accuracy of sampling-based approaches to the calculation of posterior moments, volume 196. Federal Reserve Bank of Minneapolis, Research Department Minneapolis, MN, USA, 1991.

[57] Gabriel Terejanu, Todd Oliver, and Chris Simmons. Application of predictive model selection to coupled models. arXiv preprint arXiv:1107.0927, 2011.

[58] William H Jefferys and James O Berger. Ockham's razor and bayesian analysis. American Scientist, 80(1):64-72, 1992.

[59] Phil Gibbs and Sugihara Hiroshi. What is occamâĂŹs razor. Available at: http://math.ucr.edu/home/baez/physics/General/occam.html, 1996.

[60] N. Metropolis and S. Ulam. The monte carlo method. Journal of the American Statistical Association, 4:335-341, 1949.

[61] G Peter Lepage. A new algorithm for adaptive multidimensional integration. Journal of Computational Physics, 27(2):192-203, 1978.

[62] Harold Jeffreys. The theory of probability. OUP Oxford, 1998.

[63] Julius S Bendat and Allan G Piersol. Random data: analysis and measurement procedures, volume 729. John Wiley \& Sons, 2011.

[64] Anil K Chopra. Dynamics of structures, volume 3. Prentice Hall New Jersey, 1995.

[65] John A Nelder and Roger Mead. A simplex method for function minimization. The computer journal, 7(4):308-313, 1965.

[66] C. Robert and G. Casella. Monte Carlo Statistical Methods. Springer, 2004.

[67] E. M. Hernandez and N. Polanco. Uncertainty quantification in identified modal parameters using fisher information criterion. In Proceedings of the XXXII International Modal Analysis Conference, Orlando, FL, February.

[68] Julius S Bendat and Allan G Piersol. Engineering applications of correlation and spectral analysis. New York, Wiley-Interscience, 1980. 315 p., 1, 1980. 
[69] B. R. Ellis, T. Ji, and J.D. Littler. The response of grandstands to dynamic crowd loads. Structures and Buildings, 140:355-365, 2000. 


\section{Appendix A}

\section{StABILITY CONDITIONS}

One of the most important problems in control engineering is the stability of the system. A system turns unstable when its response to a finite input turns infinite. Different methodologies has been developed in order to keep the response of the system stable [38]. In this research, the Routh's stability criterion is used to guarantee the stability of the closed-loop system modeling the human-structure interaction. The transfer function, $\mathrm{TF}(\mathrm{s})$, for a closed-loop control system, as shown in chapter 2, is:

$$
T F(s)=\frac{G(s)}{1+G(s) H(s)}
$$

By definition, the system is stable if the real part of the denominators' roots, $(1+G(s) H(s))$, are negative. This is easy to calculate for a second-order polynomial, however it becomes complex for polynomials of higher orders, such as the denominator of the systems used this research. In this chapter the stability conditions for each closed-loop control model are summarized.

\section{A.1 PID CONTROLLER}

After replacing $H(s)$ and $G(s)$ in equation 2.1, the transfer function of the system is:

$$
T F(s)=\frac{t_{i} s^{2}}{k_{p} t_{d} t_{i} s^{3}+\left(k_{p} t_{i}+m t_{i}\right) s^{2}+\left(k_{p}+t_{i} c\right) s+t_{i} k}
$$

Therefore, following the Routh's stability criterion, the system becomes stable if:

- $k_{p} t_{d} t_{i}>0$ 
- $k_{p} t_{i}+m t_{i}>0$

- $k_{p}+t_{i} c>0$

- $t_{i} k>0$

- $t_{i}\left[\left(k_{p}+m\right)\left(k_{p}+t_{i} c\right)\right]>k_{p} t_{d} t_{i}^{2} k$

\section{A.2 PI CONTROLler}

After replacing $H(s)$ and $G(s)$ in equation 2.1, the transfer function of the system is:

$$
T F(s)=\frac{t_{i} s^{2}}{t_{i}\left(k_{p}+m\right) s^{2}+\left(k_{p}+t_{i} c\right) s+t_{i} k}
$$

Therefore, following the Routh's stability criterion, the system becomes stable if:

- $\left(k_{p}+m\right) t_{i}>0$

- $k_{p}+t_{i} c>0$

- $t_{i} k>0$

\section{A.3 PD CONTROLler}

After replacing $H(s)$ and $G(s)$ in equation 2.1, the transfer function of the system is:

$$
T F(s)=\frac{s^{2}}{k_{p} t_{d} s^{3}+\left(k_{p}+m\right) s^{2}+c s+k}
$$

Therefore, following the Routh's stability criterion, the system becomes stable if:

- $k_{p} t_{d}>0$

- $k_{p}+m>0$

- $\left(k_{p}+m\right) c>\left(k_{p} t_{d}\right) k$ 


\section{ApPendix B}

\section{Convergence}

The convergence of the parameters of each model is checked by the Geweke z-score $[56]$.

\section{B.1 Models of The Structure}

B.1.1 MCK MOdEL OF THE STRUCTURE

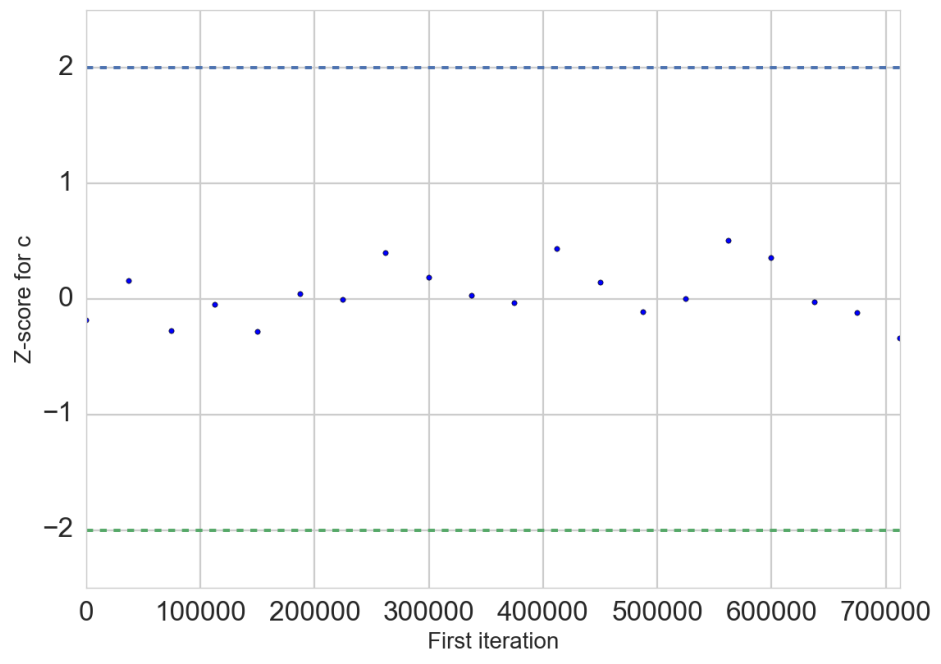

Figure B.1: Test 1 


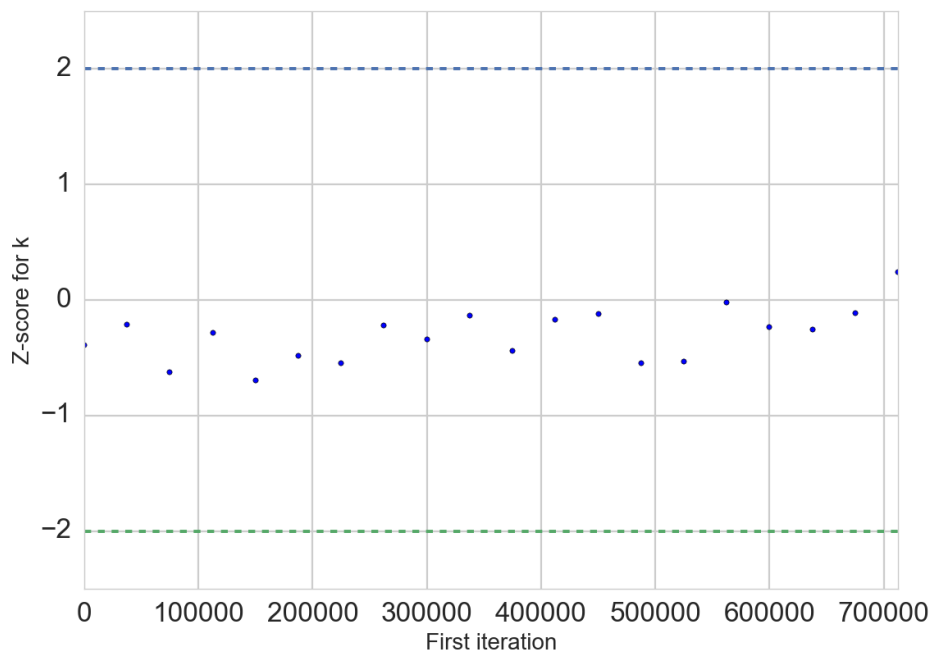

Figure B.2: Test 2

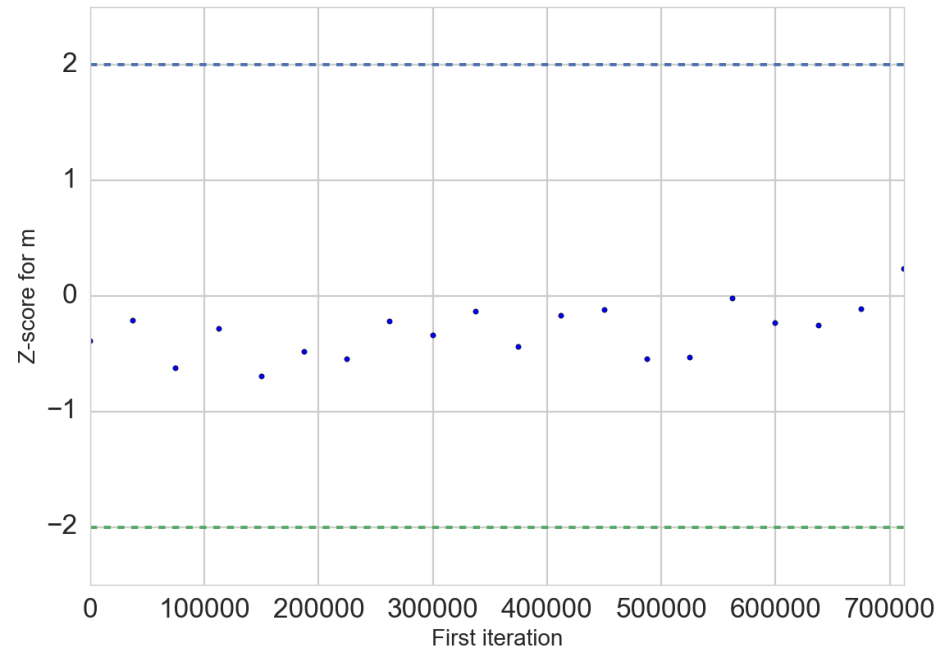

Figure B.3: Test 3 


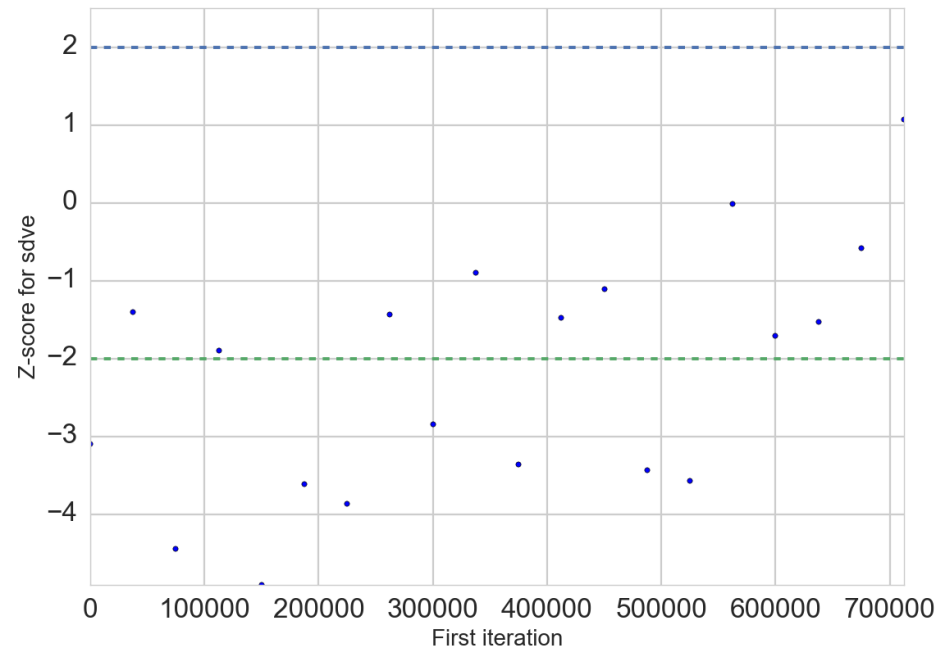

Figure B.4

\section{B.1.2 Poles And Zeros model of the Structure}

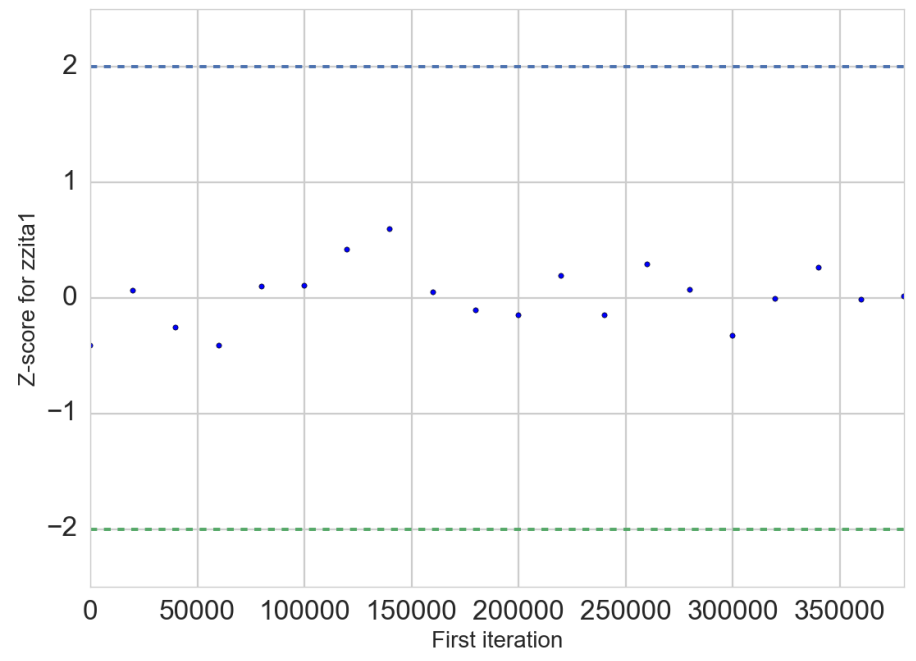

Figure B.5 


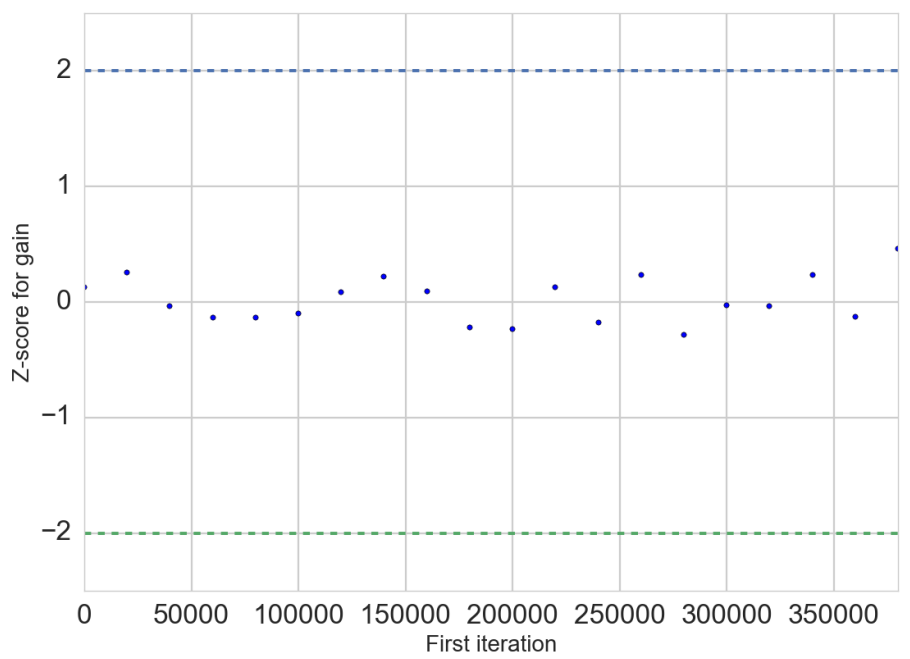

Figure B.6

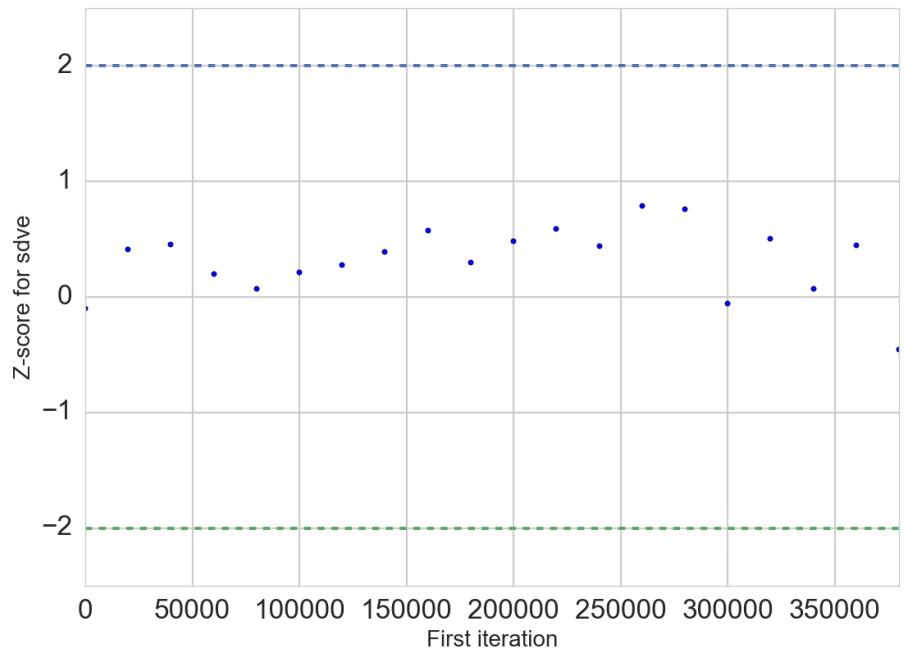

Figure B.7 


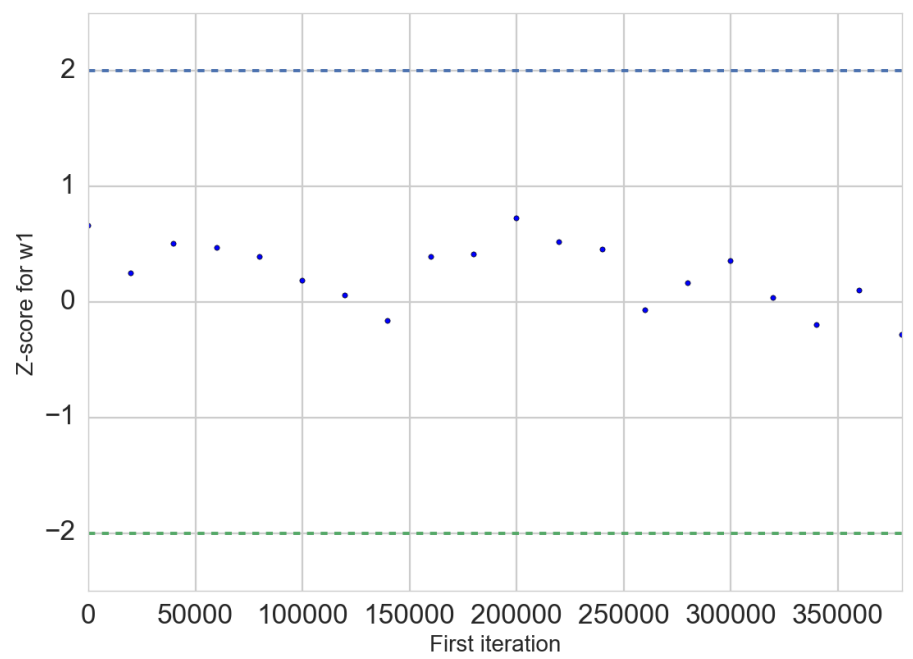

Figure B.8

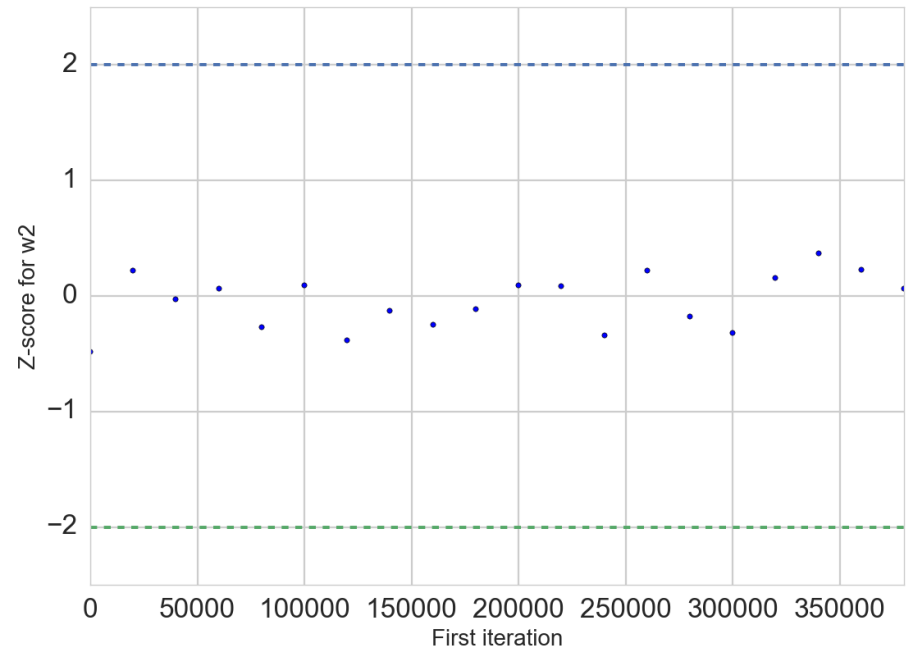

Figure B.9 


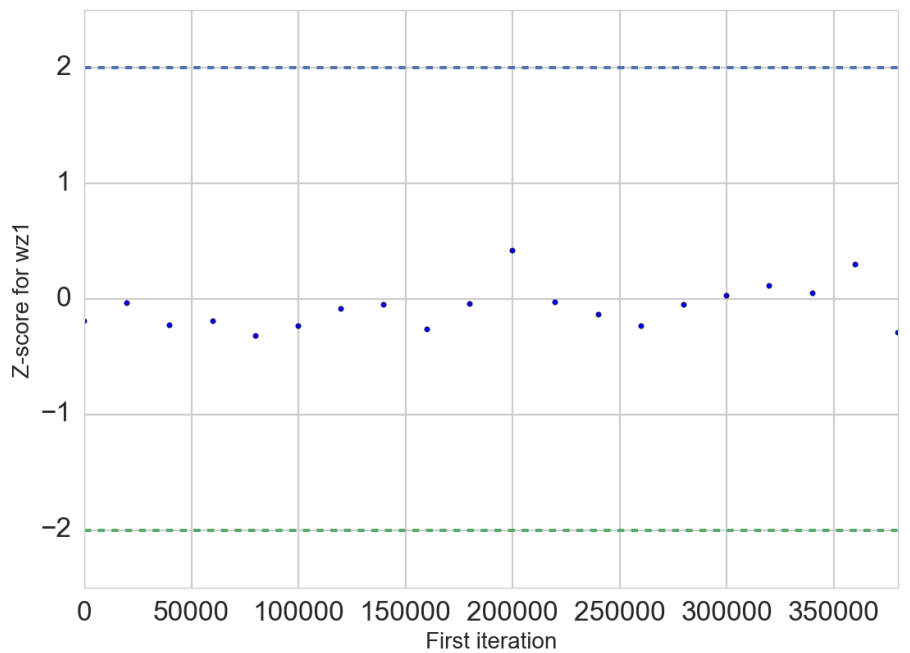

Figure B.10

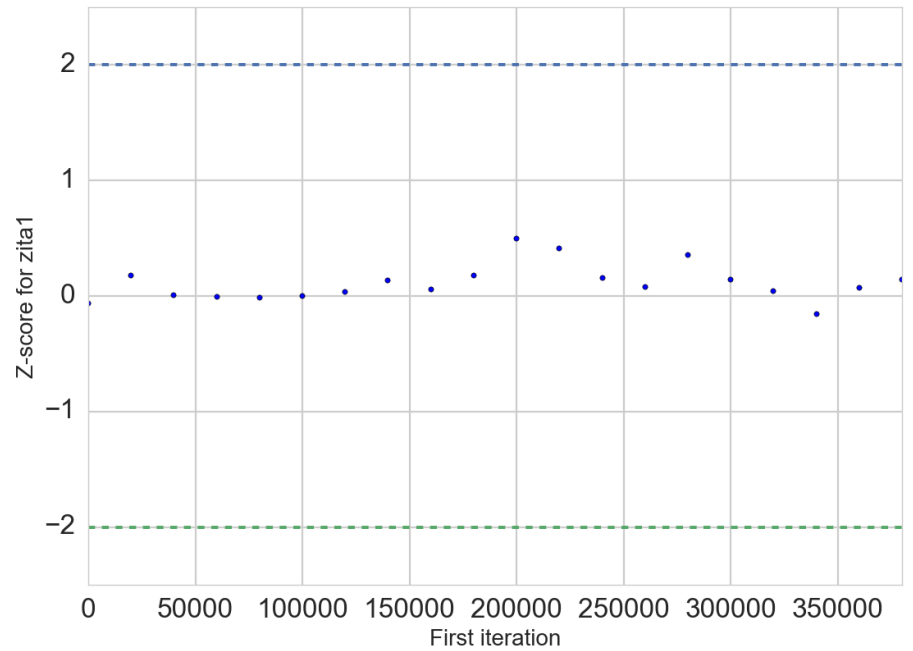

Figure B.11 


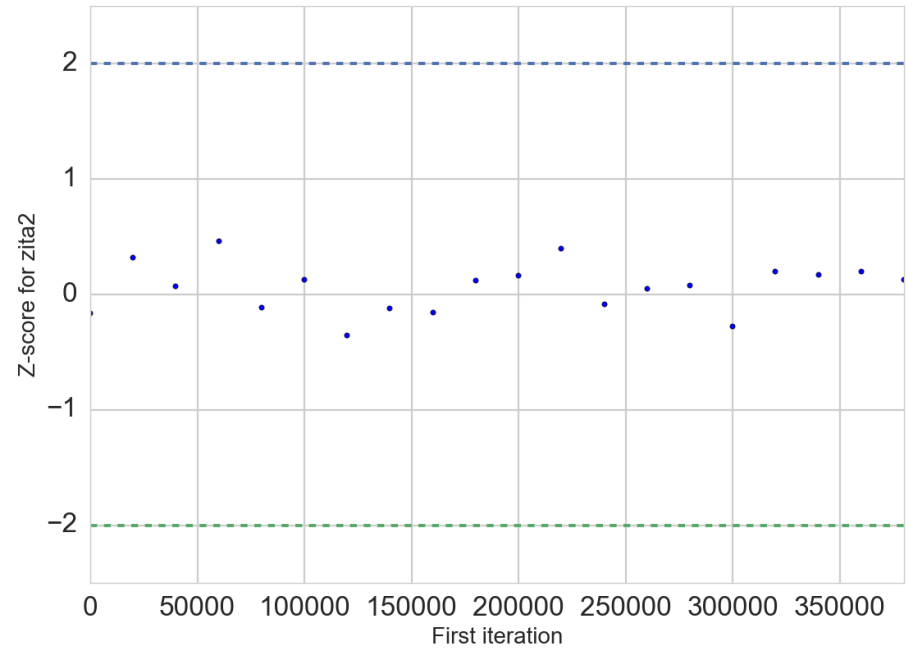

Figure B.12

\section{B.2 MOdels OF HUMAN-STRUCTURE INTERACTION}

\section{B.2.1 PID CONTROLLER}

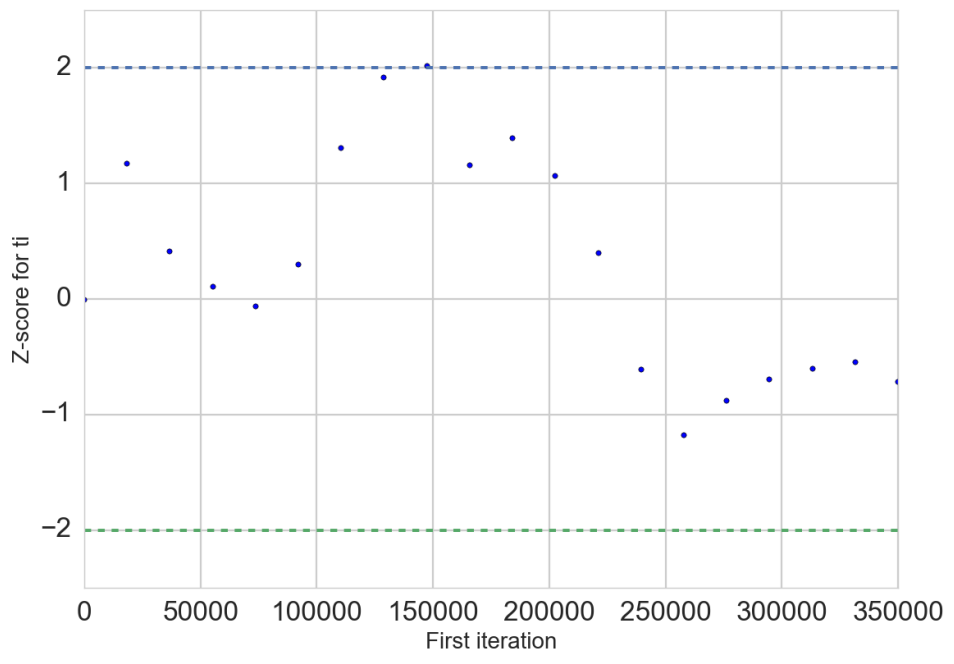

Figure B.13 


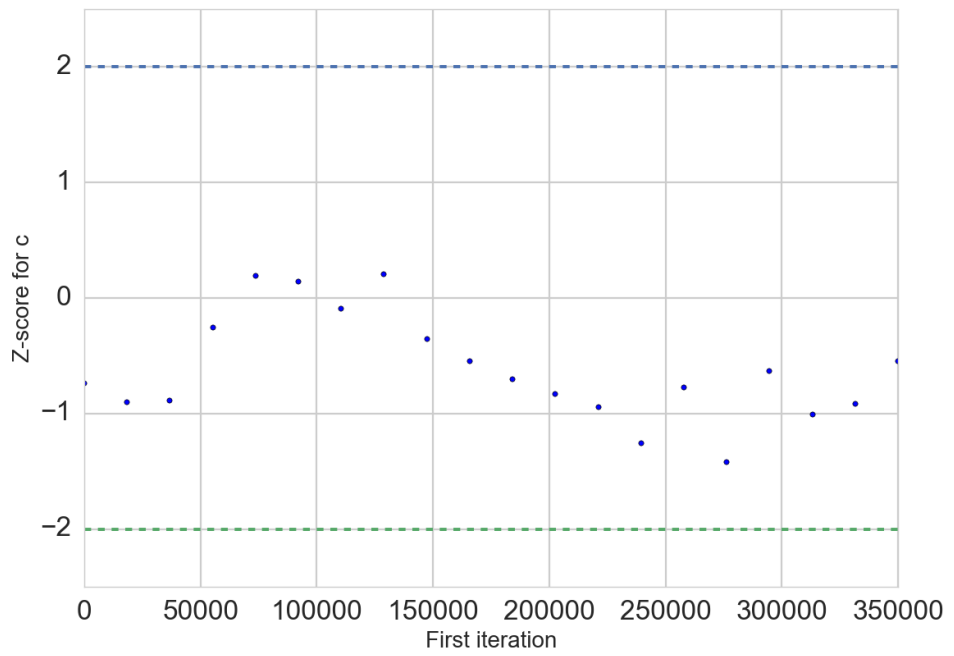

Figure B.14

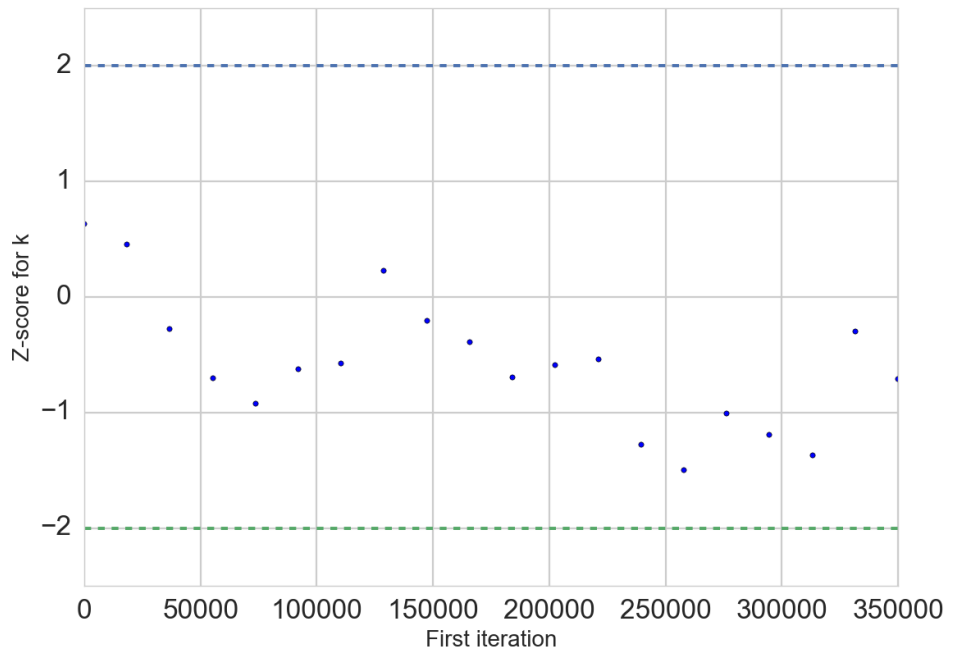

Figure B.15 


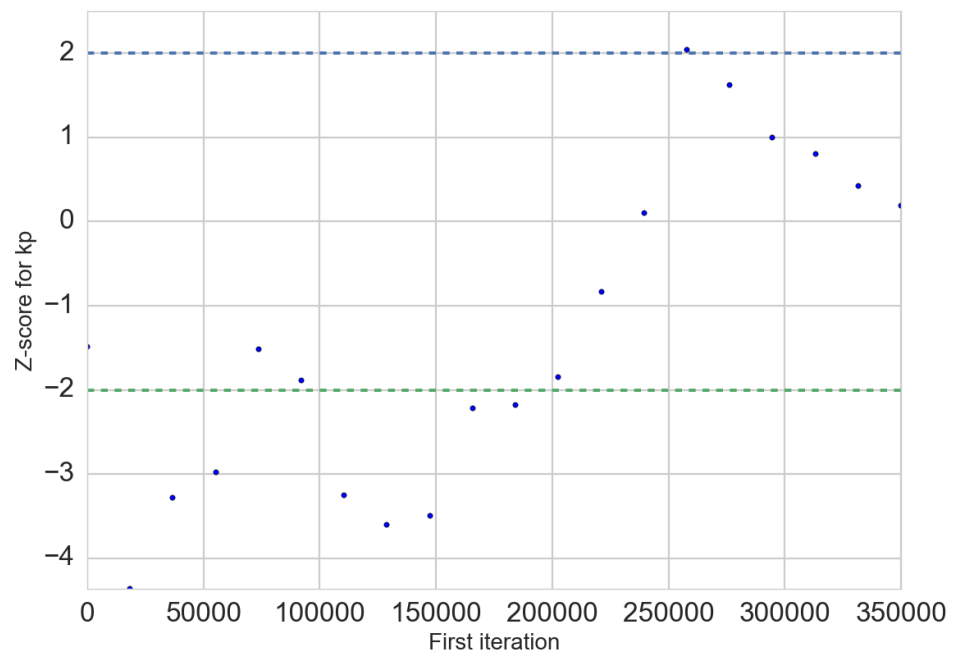

Figure B.16

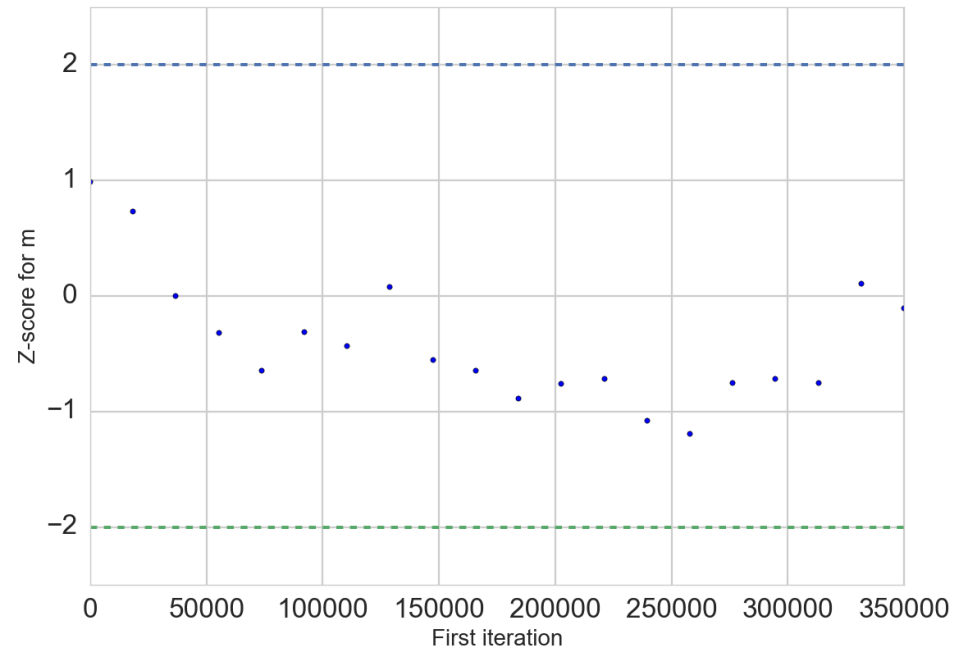

Figure B.17 


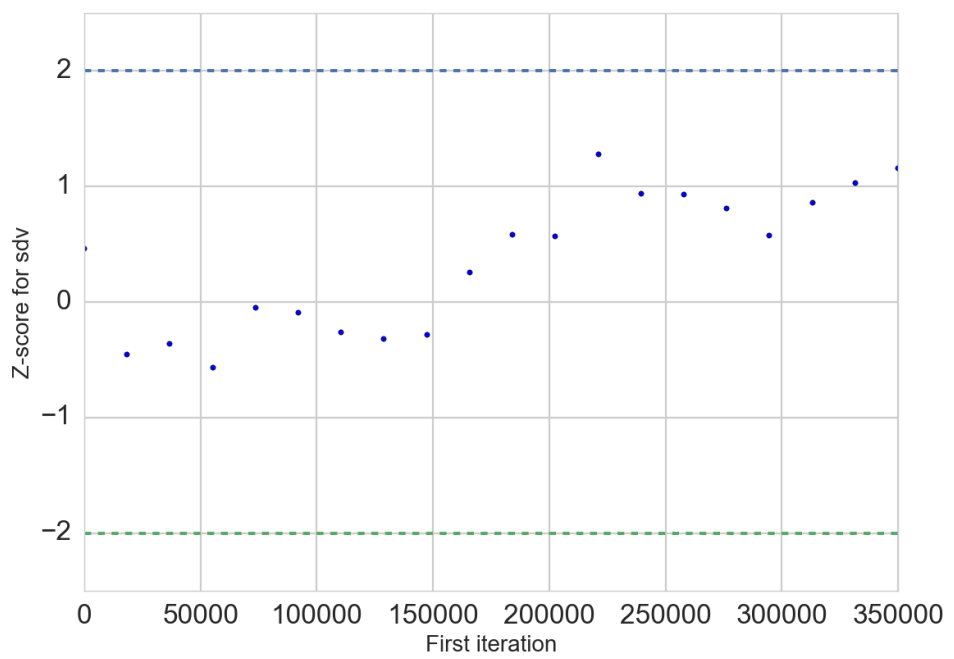

Figure B.18

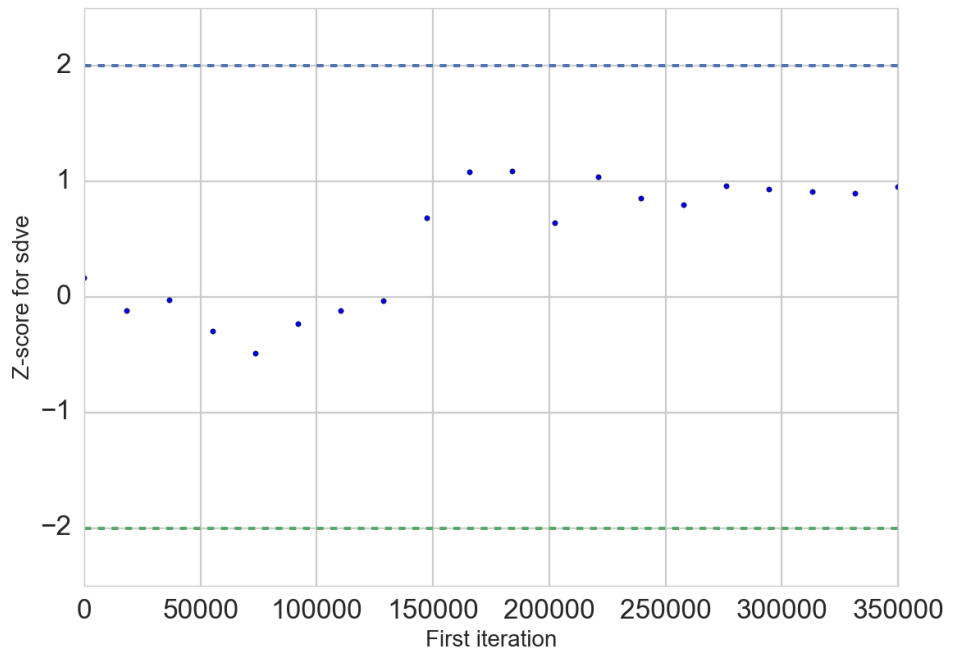

Figure B.19 


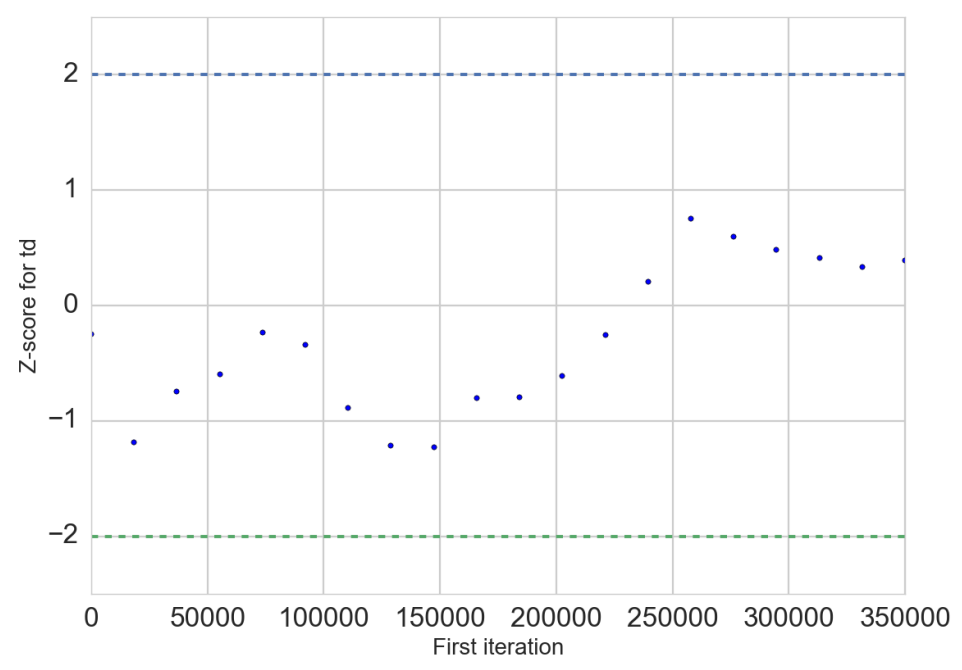

Figure B.20

\section{B.2.2 PI CONTROLler}

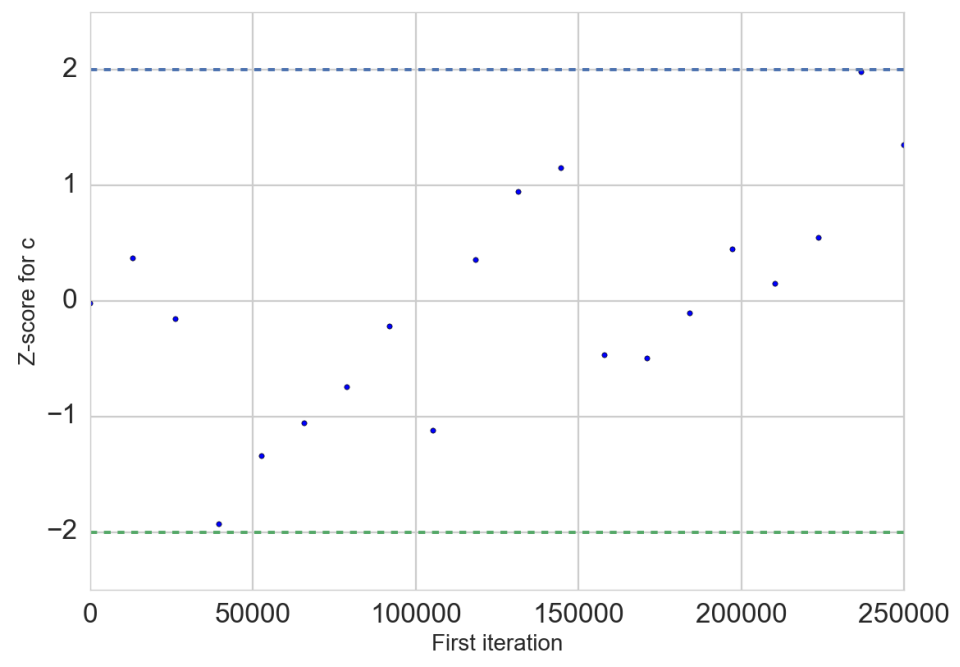

Figure B.21 


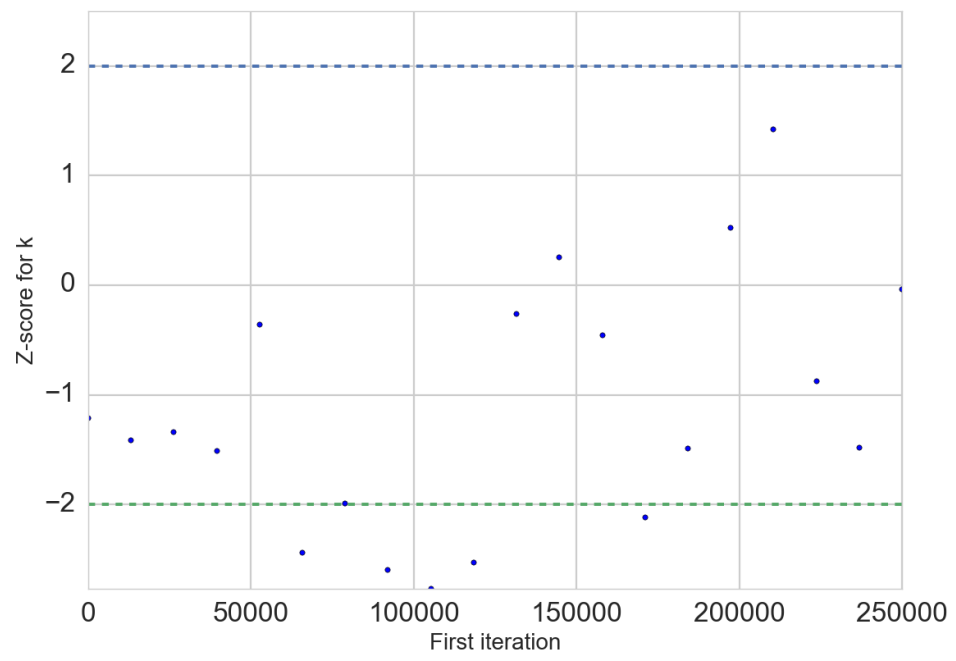

Figure B.22

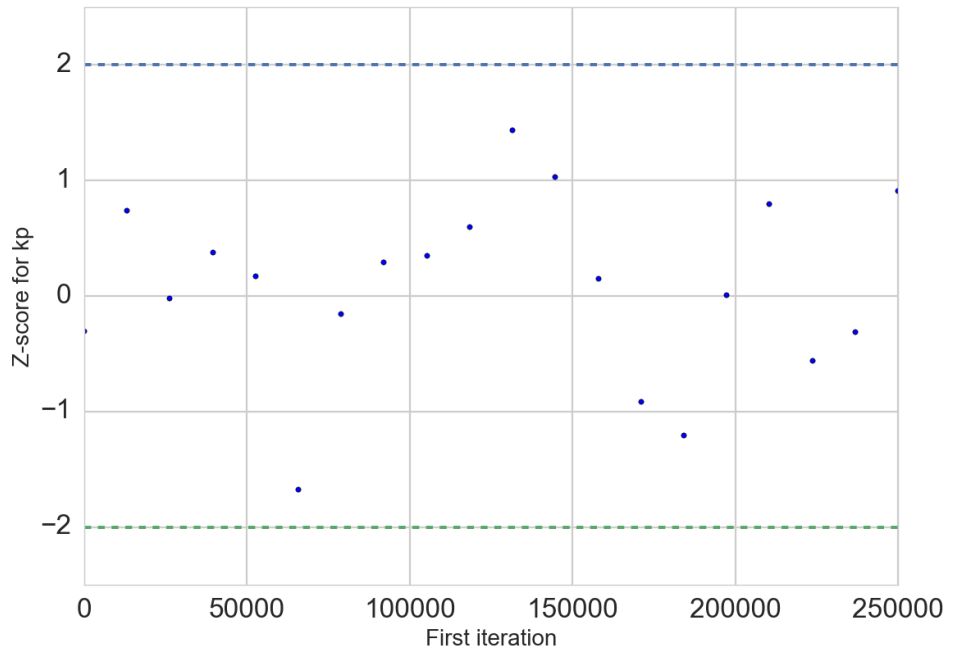

Figure B.23 


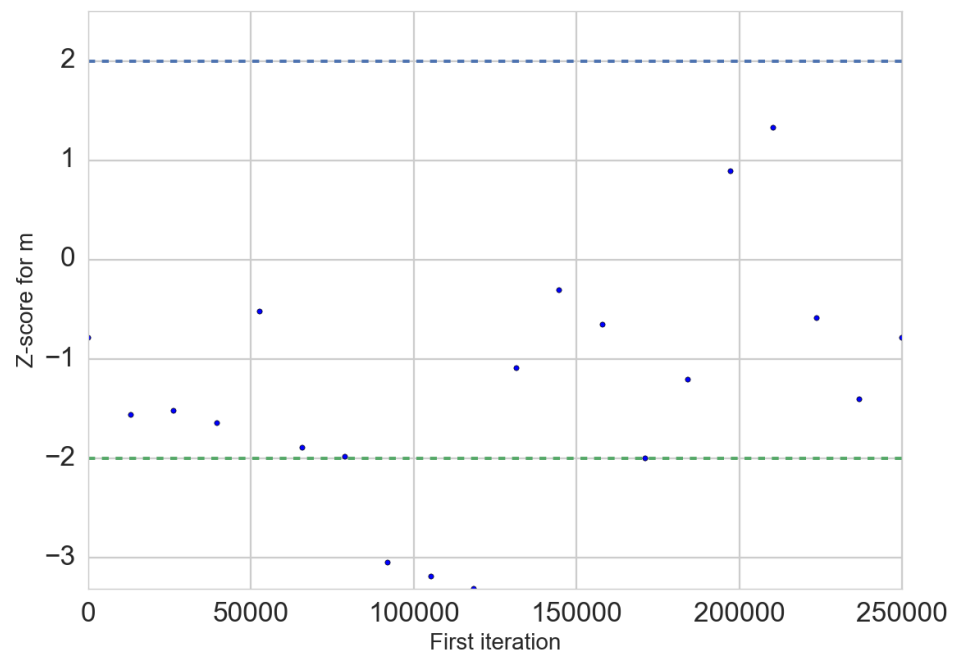

Figure B.24

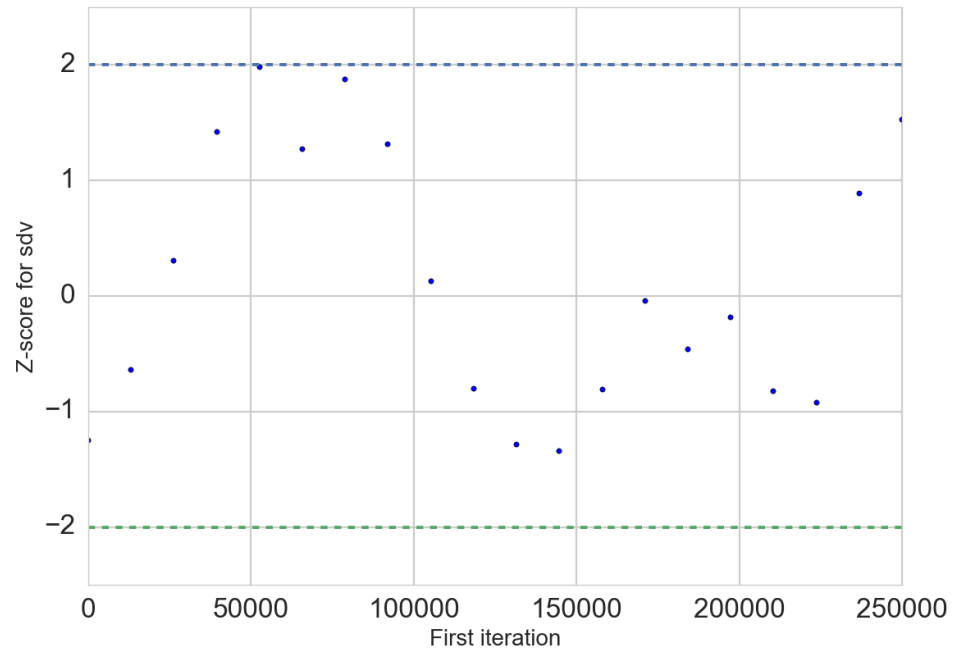

Figure B.25 


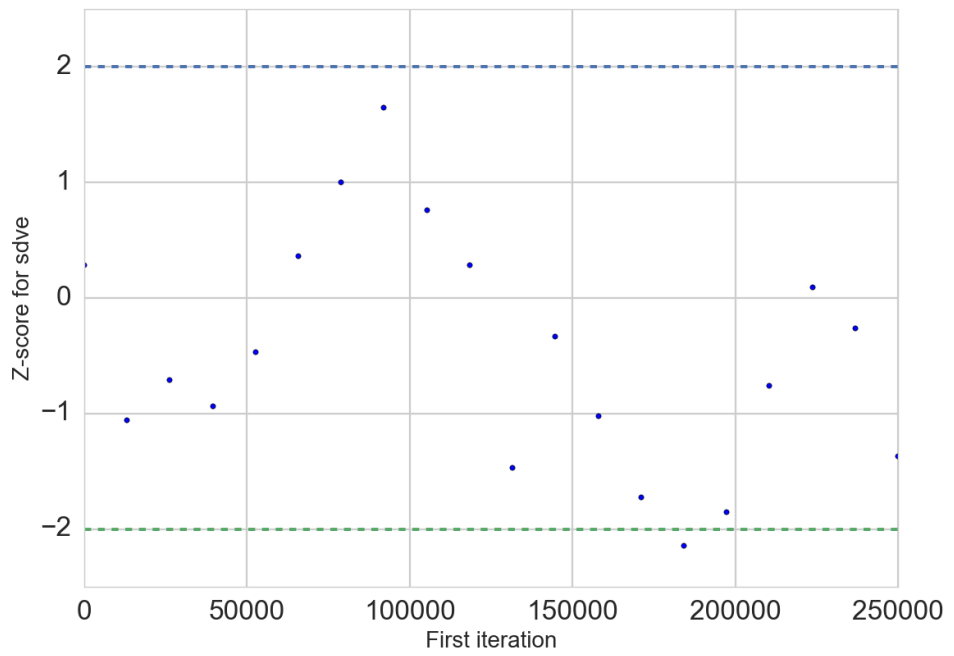

Figure B.26

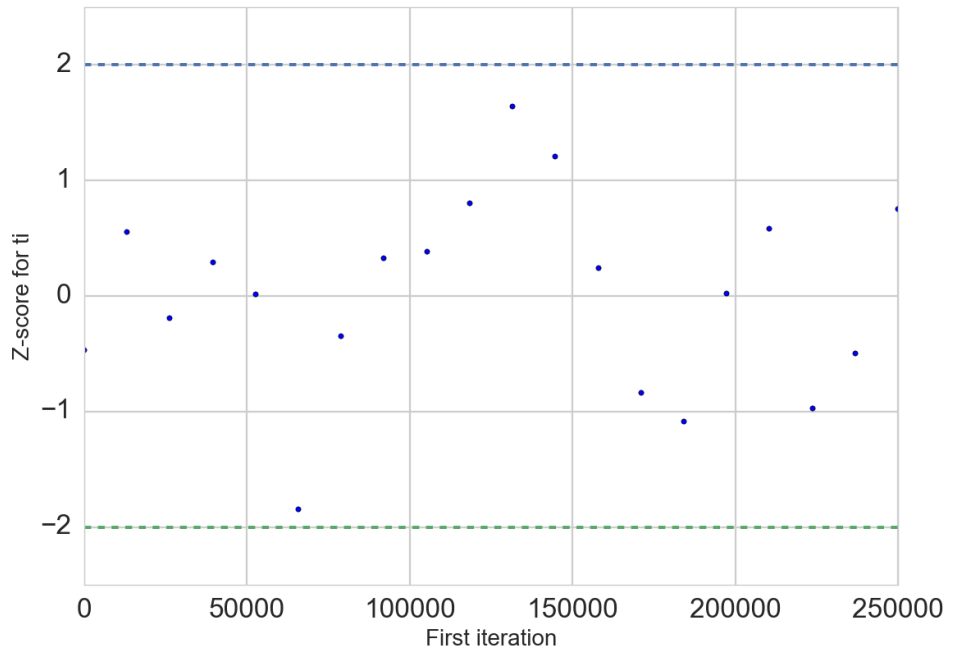

Figure B.27 


\section{B.2.3 PD CONTROLleR}

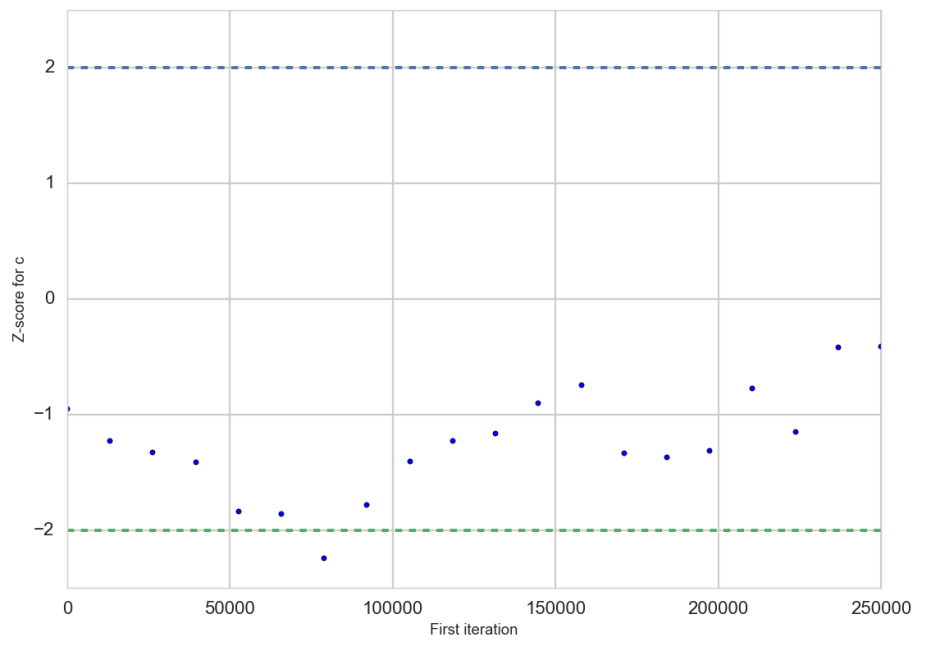

Figure B.28 


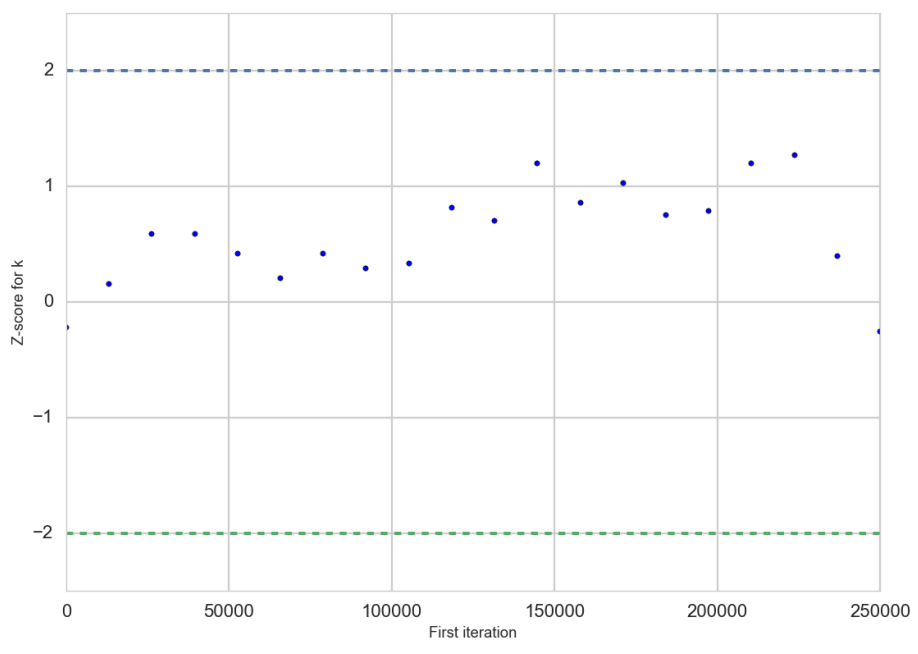

Figure B.29

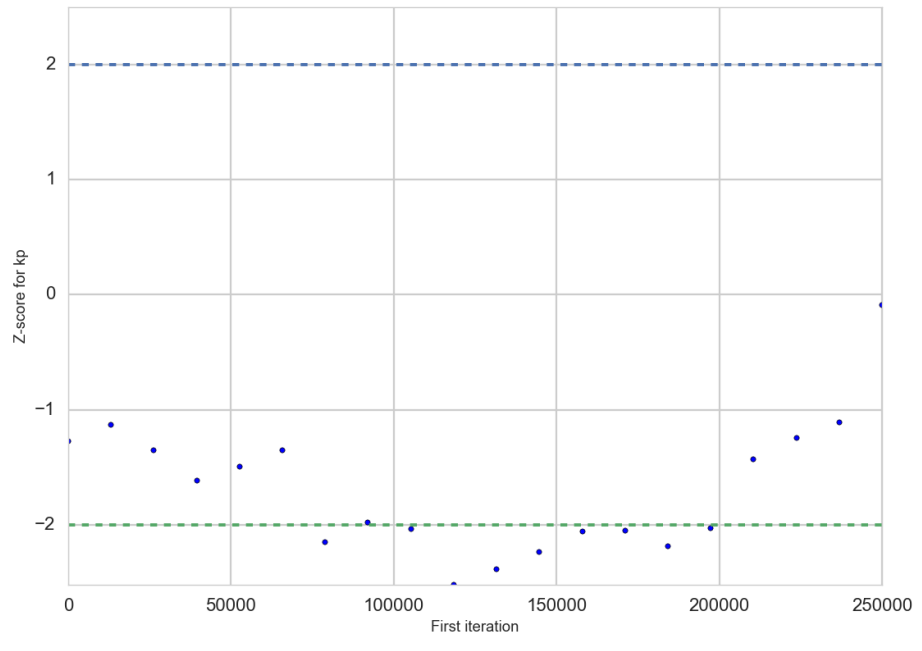

Figure B.30 


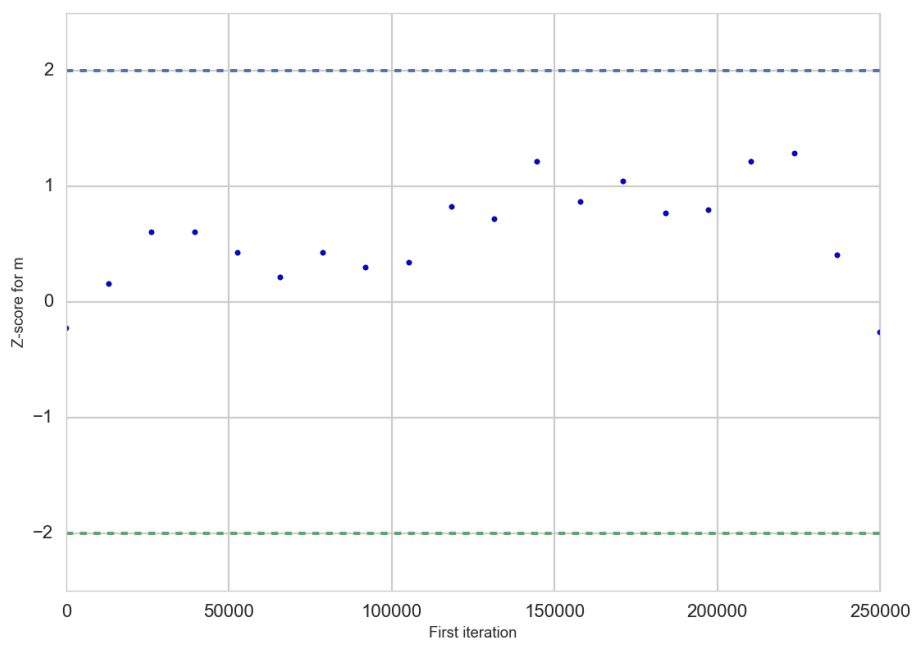

Figure B.31

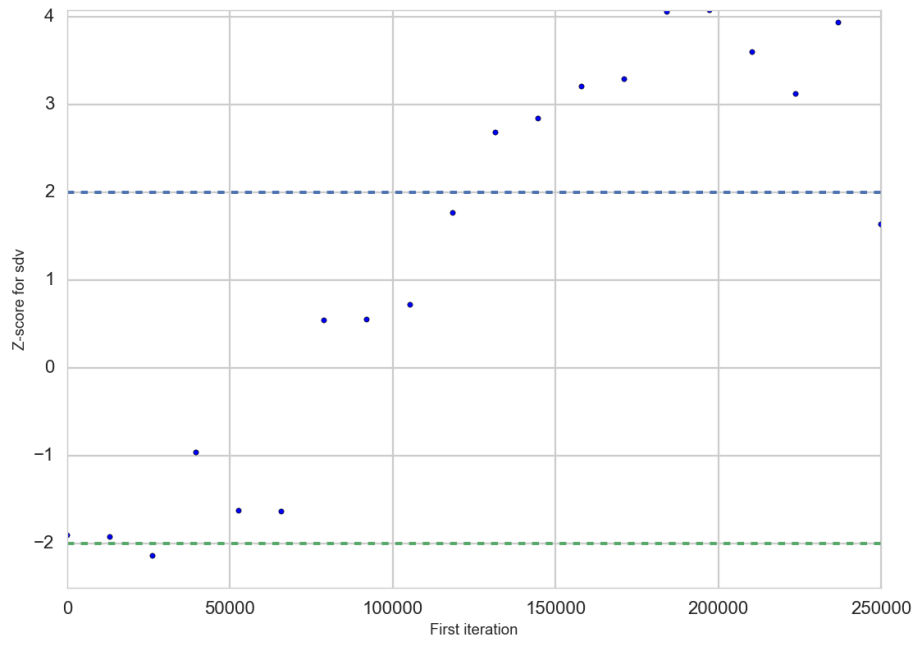

Figure B.32 


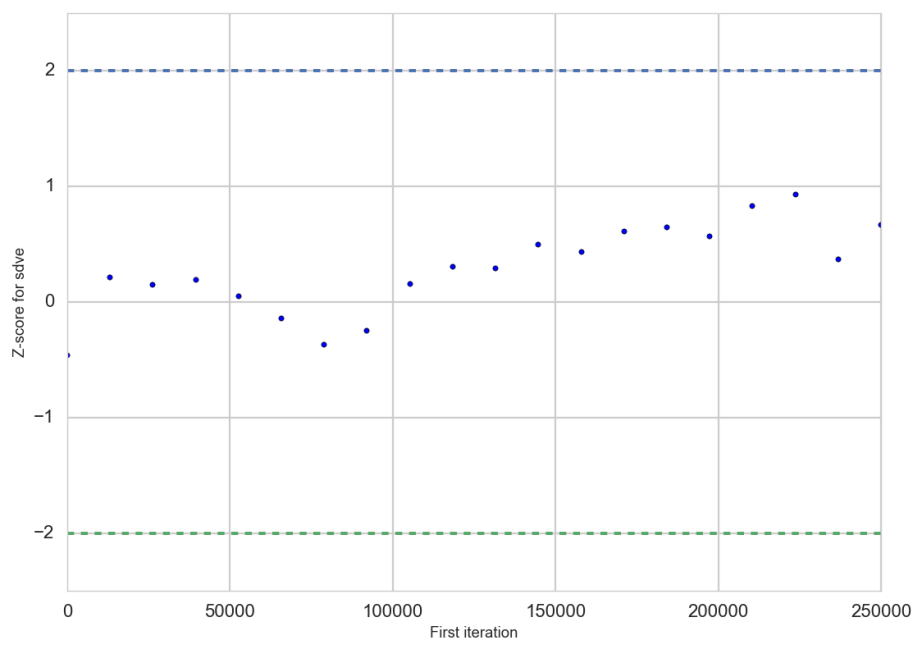

Figure B.33

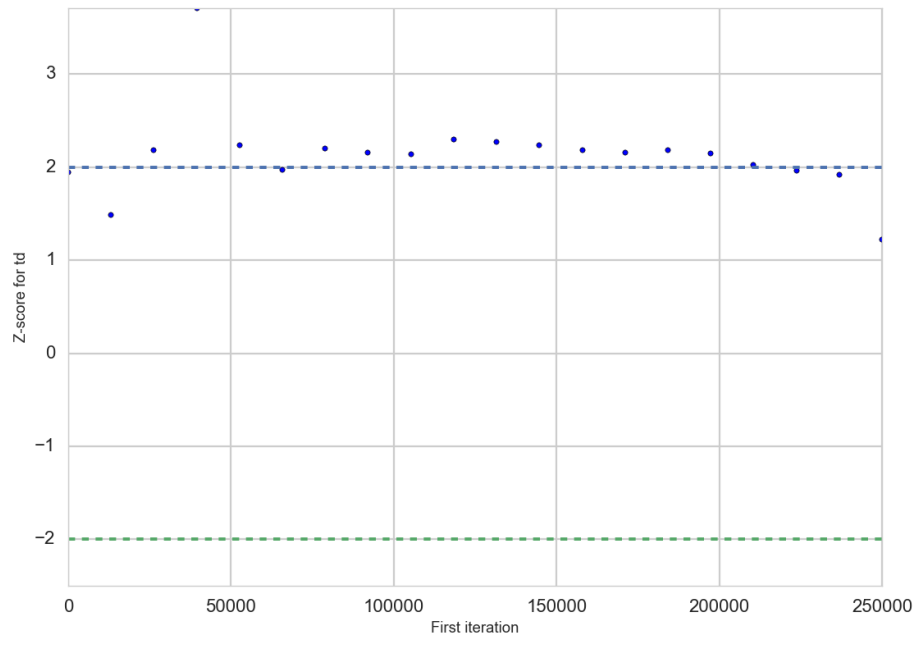

Figure B.34 


\section{B.2.4 SDOF MODEL OF THE HUMAN}

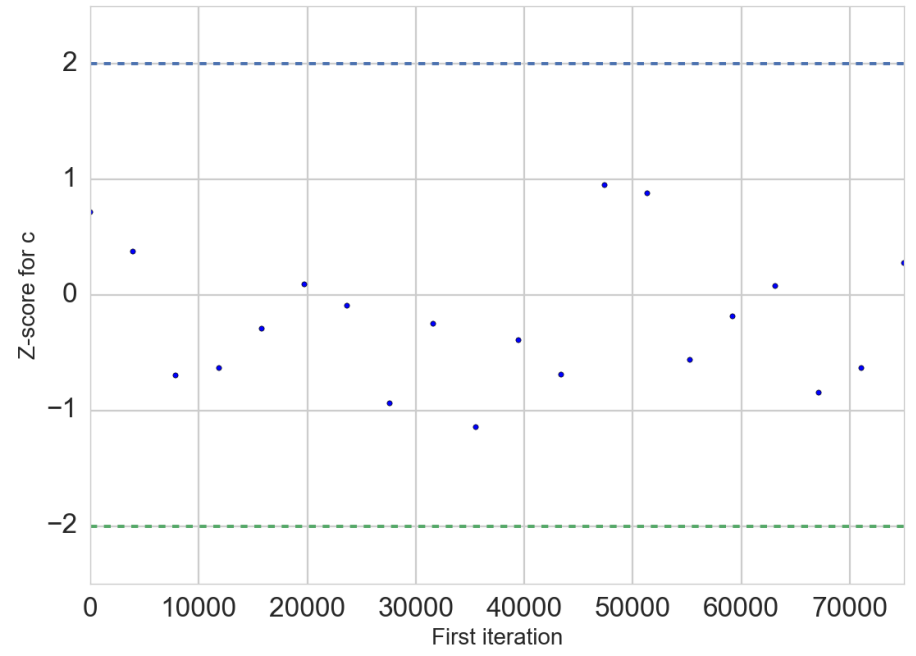

Figure B.35 


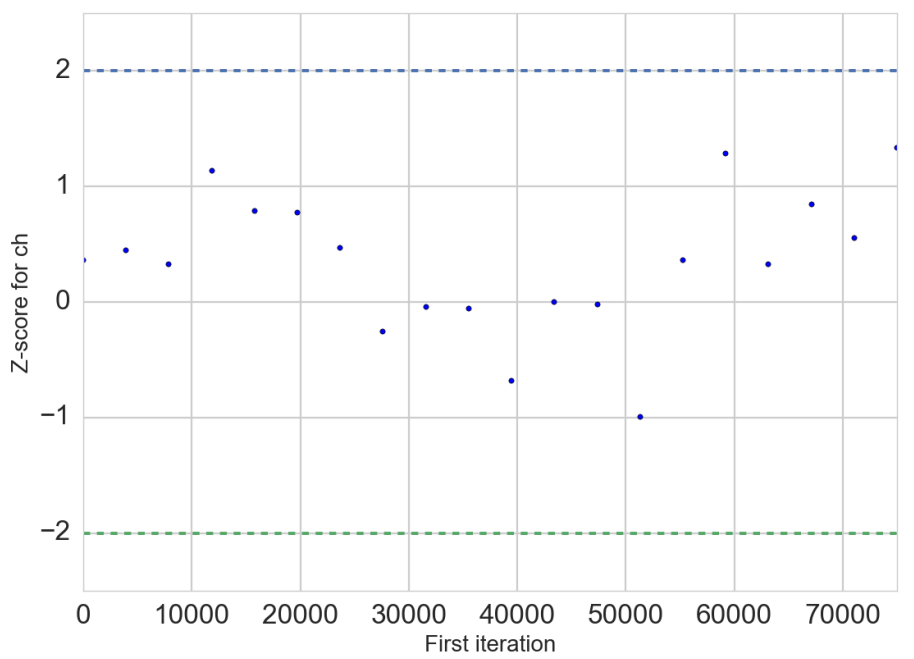

Figure B.36

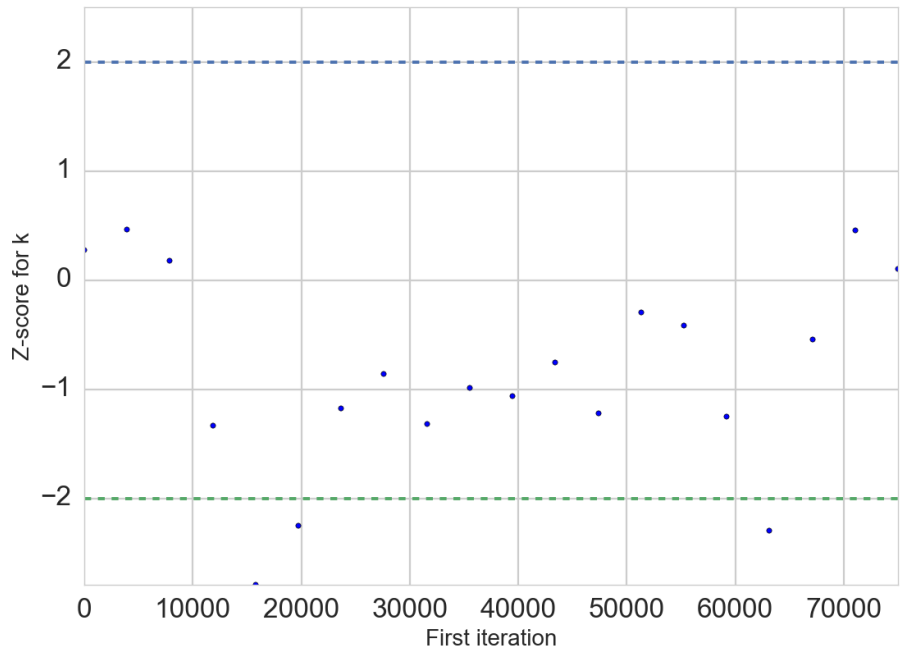

Figure B.37 


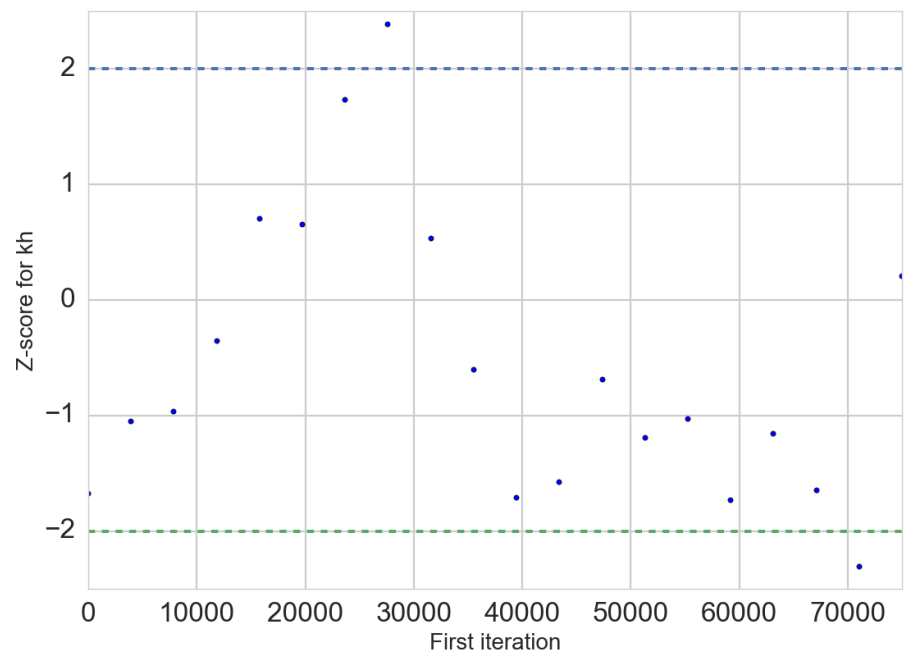

Figure B.38

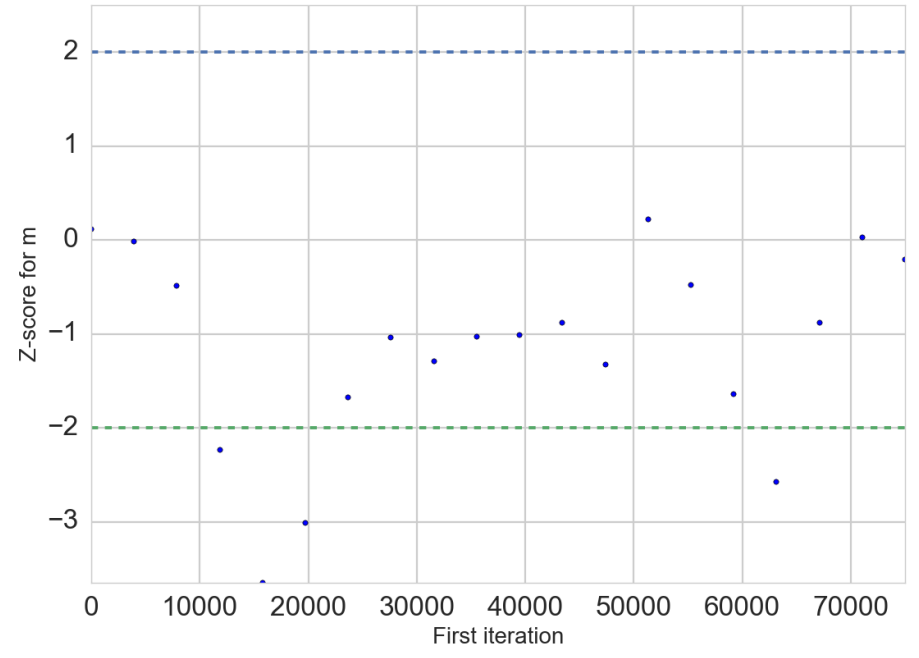

Figure B.39 


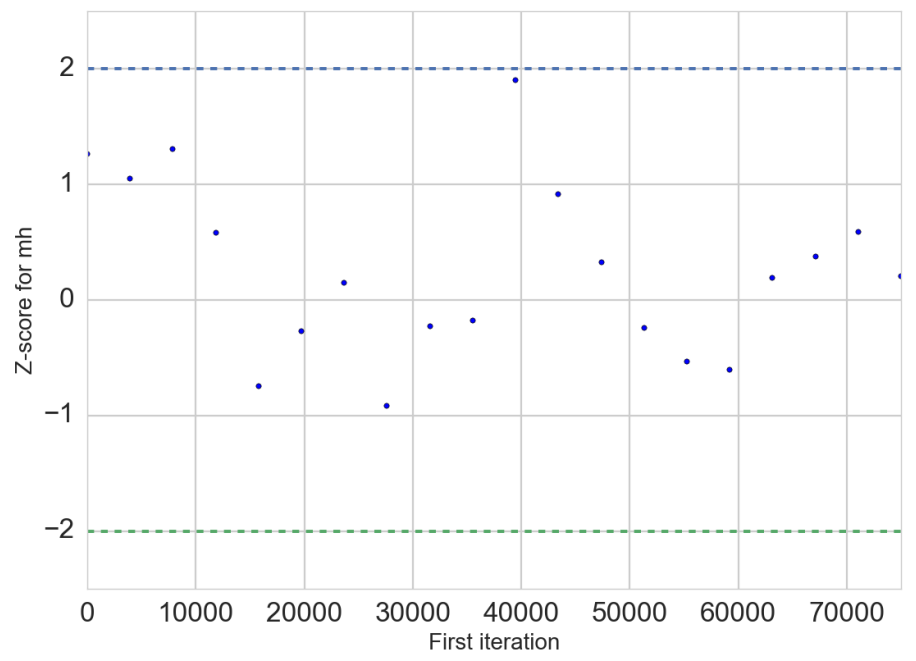

Figure B.40

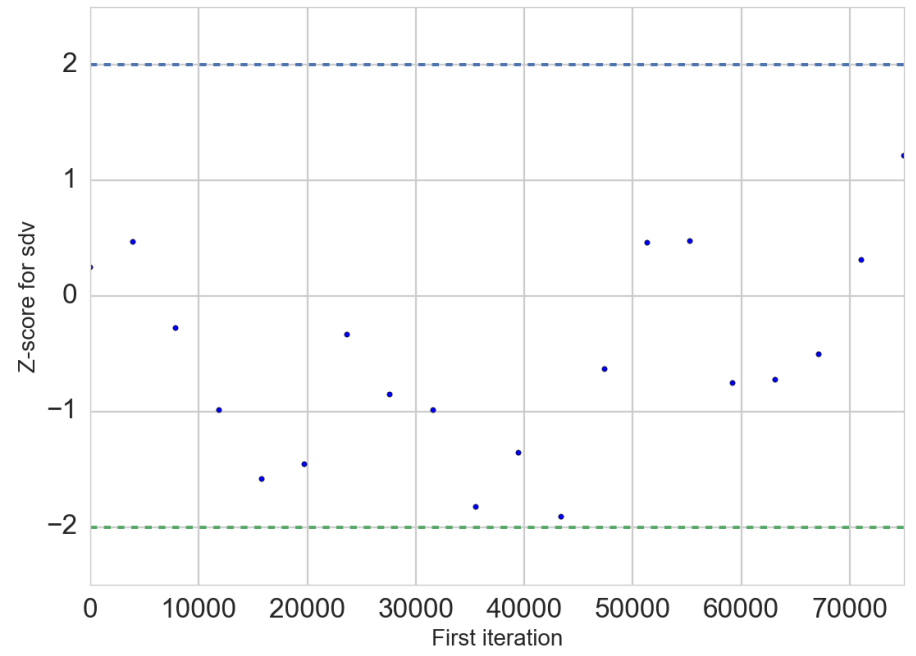

Figure B.41 


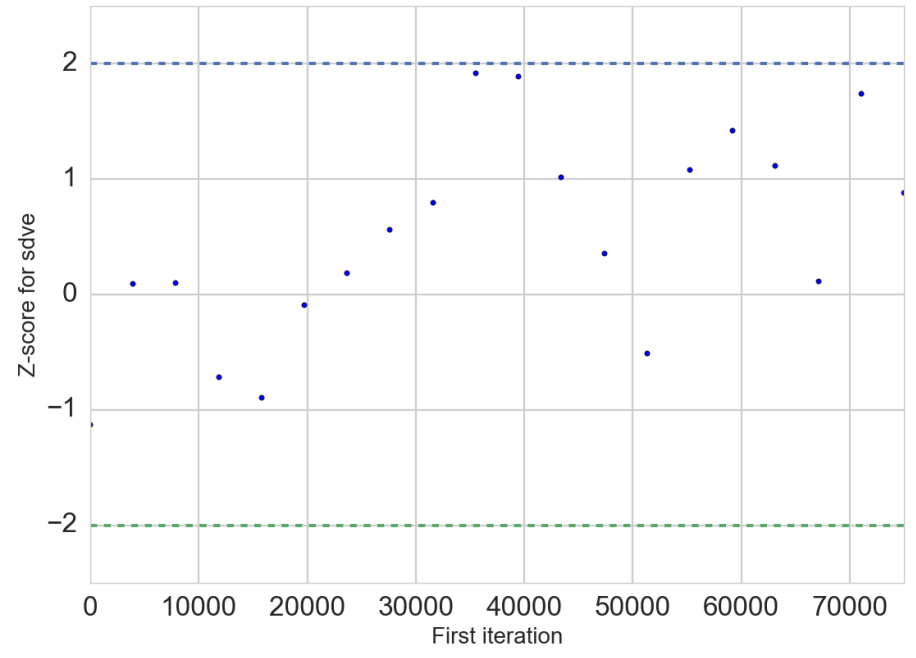

Figure B.42

\section{B.2.5 Two DOF CONTRoller}

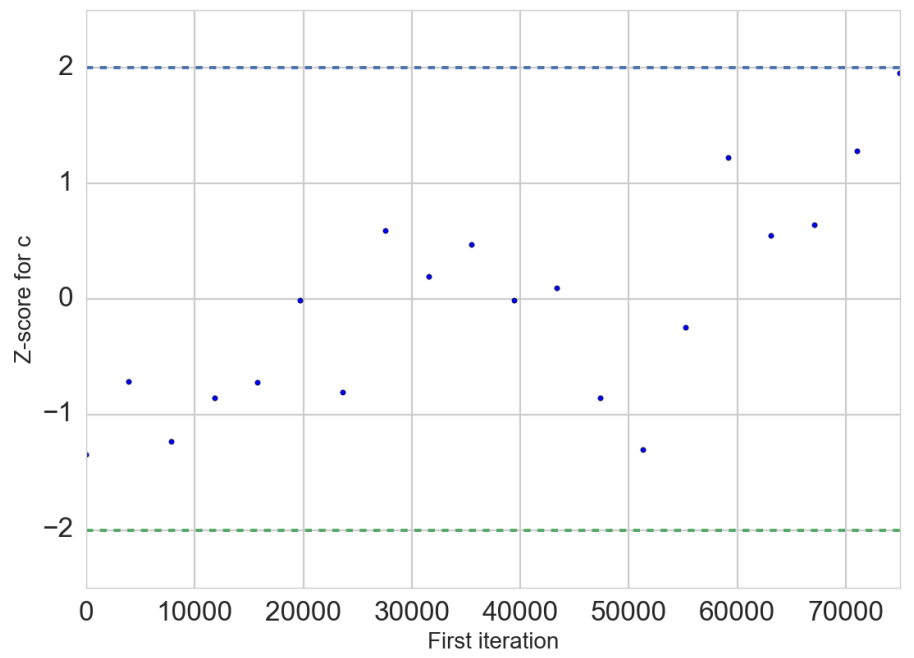

Figure B.43 


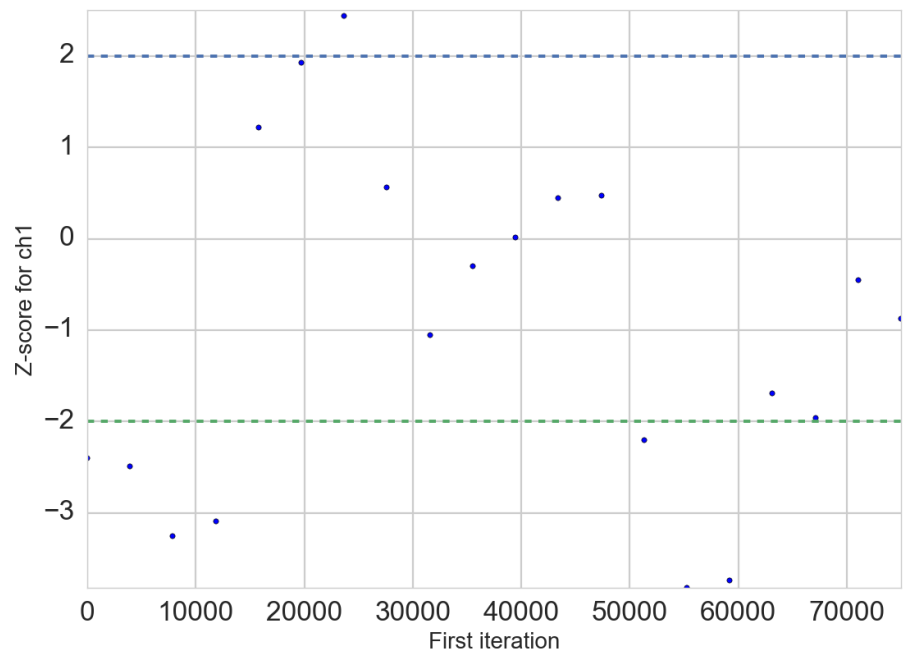

Figure B.44

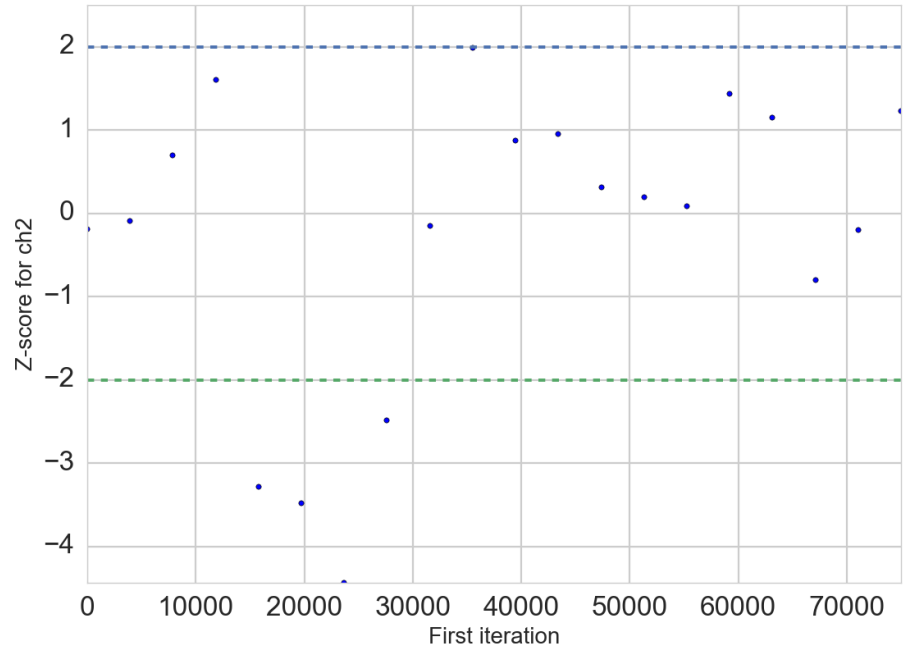

Figure B.45 


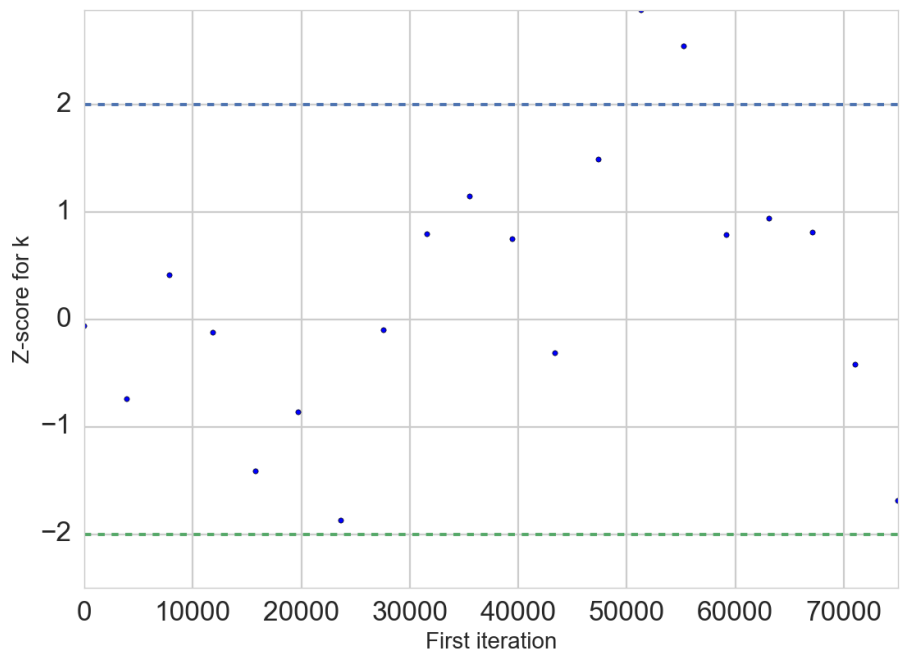

Figure B.46

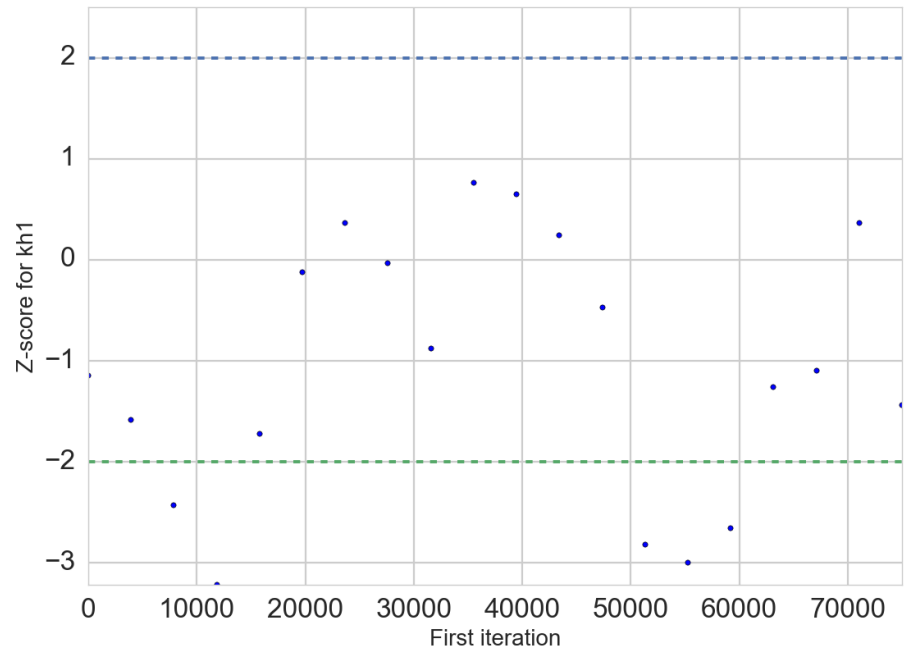

Figure B.47 


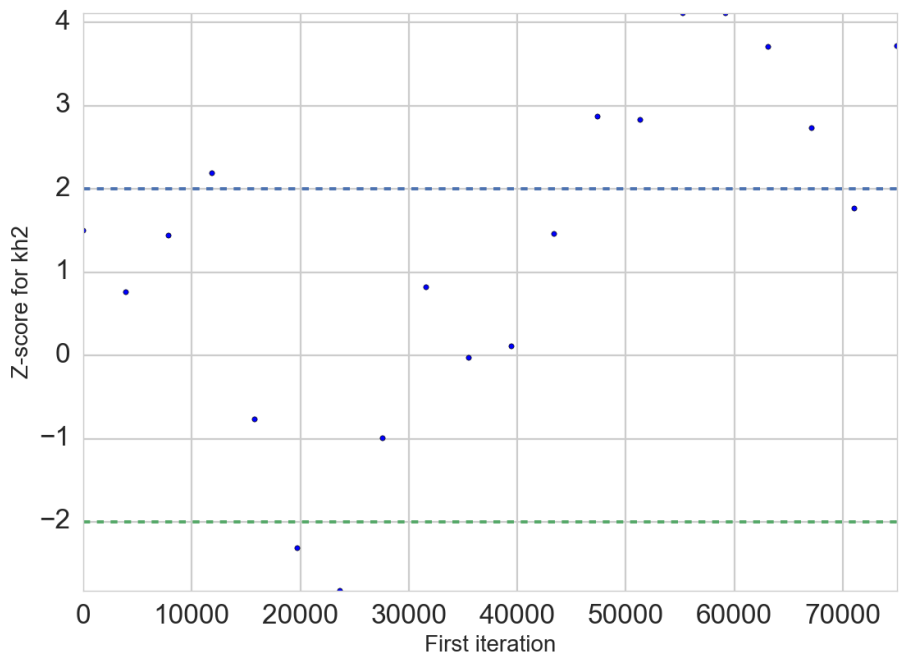

Figure B.48

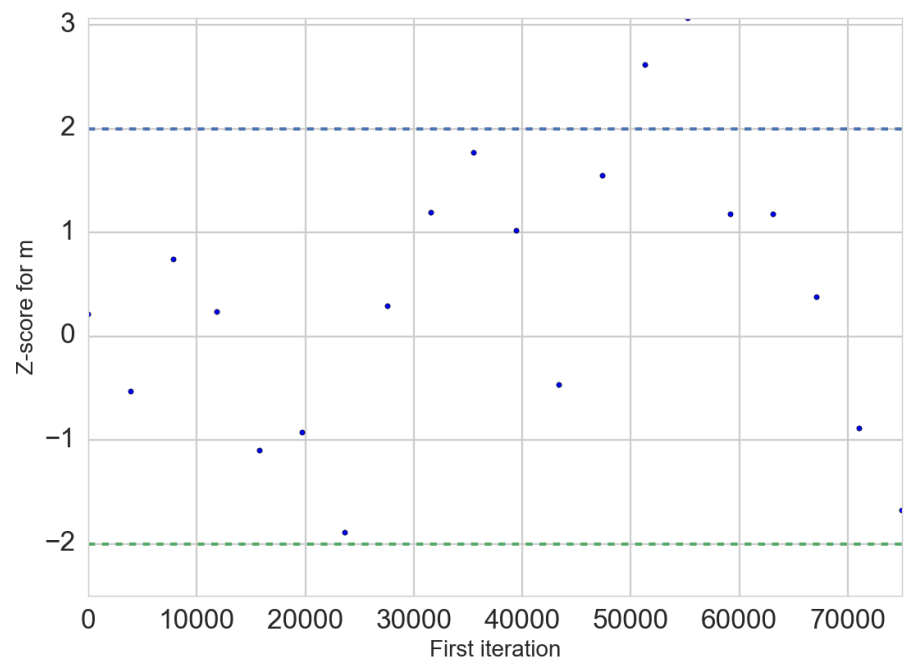

Figure B.49 


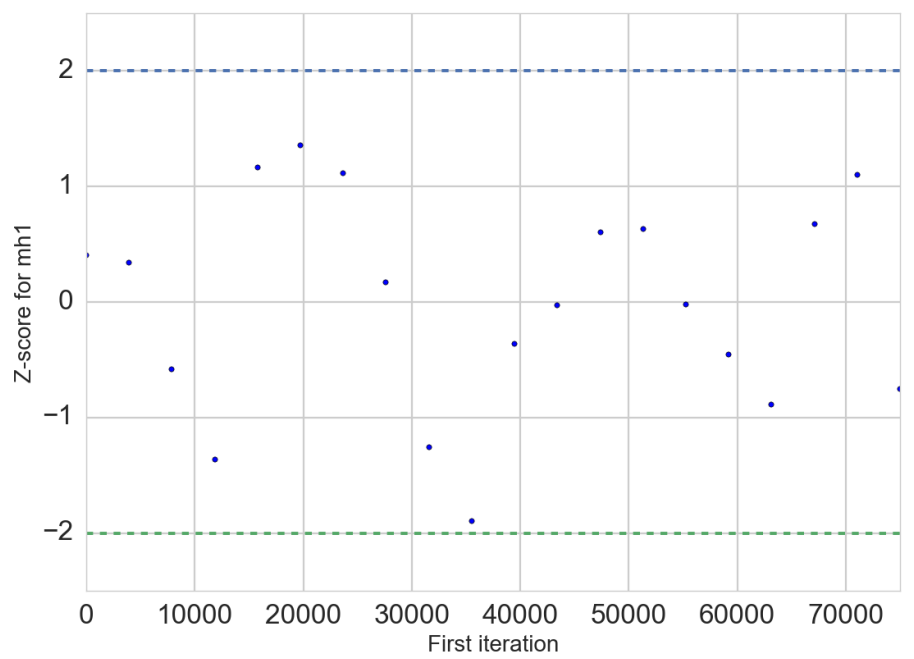

Figure B.50

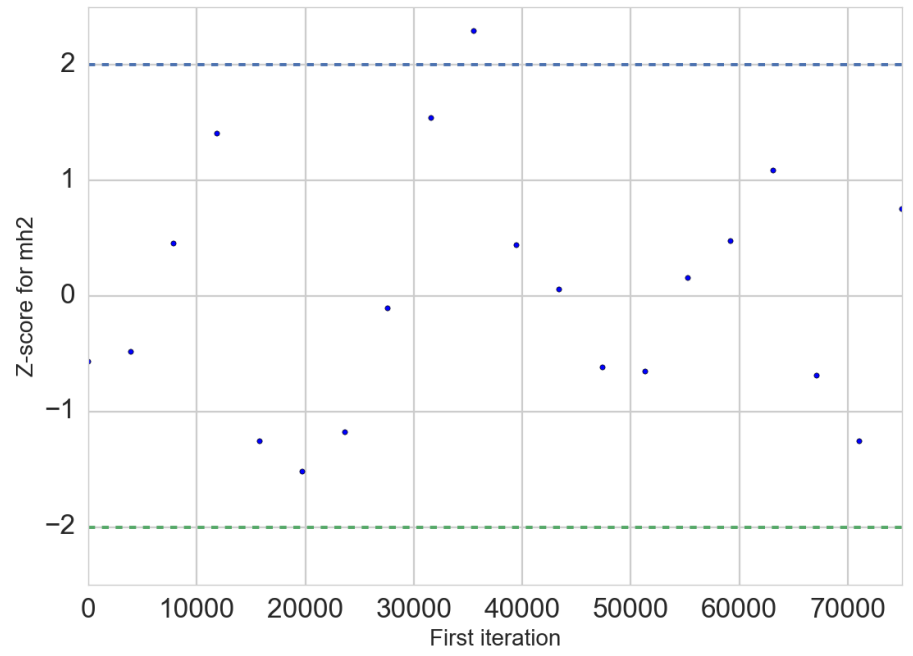

Figure B.51 


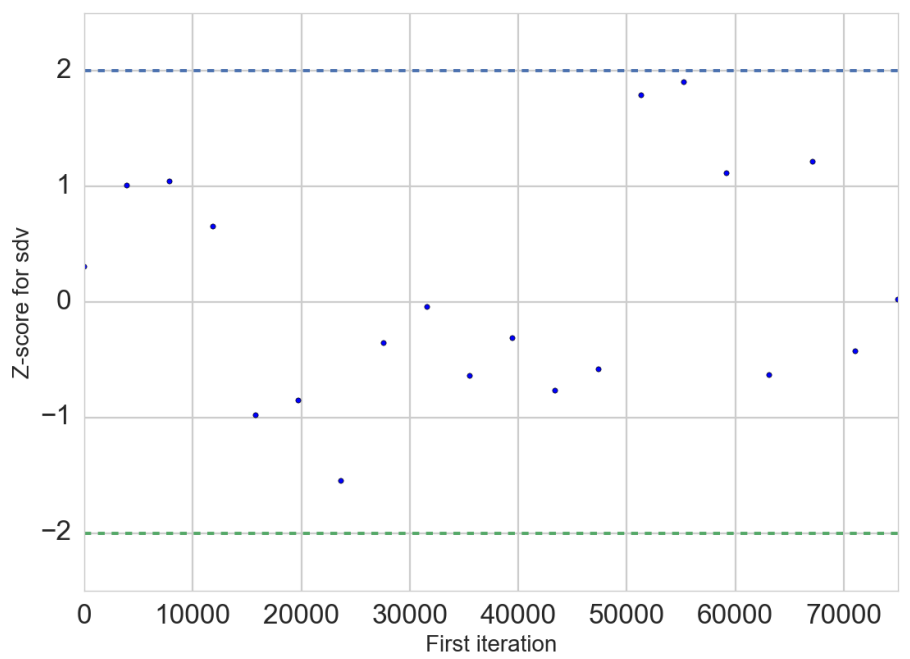

Figure B.52

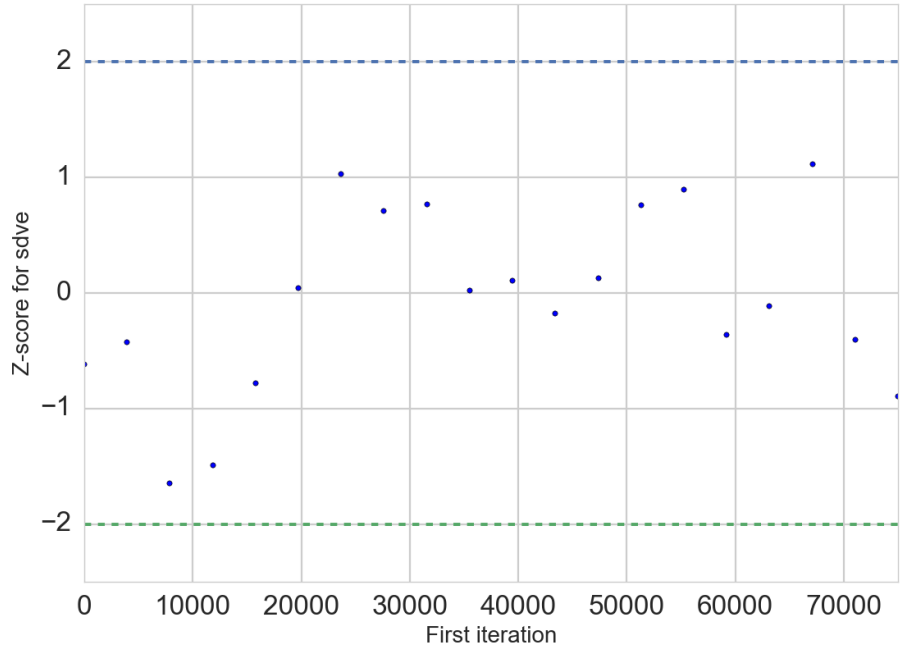

Figure B.53 